\title{
Investigation of Reduced-Order Modeling for Aircraft Stability and Control Prediction
}

\author{
Neal T. Frink*, Brett R. Hiller ${ }^{\dagger}$, Patrick C. Murphy ${ }^{\ddagger}$, Kevin Cunningham ${ }^{\S}$, and Gautam H. Shah ${ }^{\mathbb{I}}$ \\ NASA Langley Research Center, Hampton, VA, 23681
}

High-fidelity computational fluid dynamics tools offer the potential to approximate increments for ground-to-flight scaling effects, as well as to augment the dynamic damping derivative data for motion-based flight simulators. Unfortunately, the computational expense is currently prohibitive for populating a complete simulator database. This work investigates an existing surrogate-based, indicial response reduced-order model methodology as a means to efficiently augment a flight simulator database with high-fidelity nonlinear aerodynamic damping derivatives. Creation of the reduced-order model is based on the superposition integrals of the step response with the derivative of its corresponding input signal. Step responses are calculated using a computational grid motion approach that separates the effects of angle of attack and sideslip angle from angular rates, and rates from angle of attack and sideslip. It is demonstrated that the transients produced during the start of a forced-oscillation motion are captured by the reduced-order model to the level of fidelity of a comparable computational solution. Aerodynamic coefficients computed within minutes by the reduced-order model for an aircraft undergoing an 18-second half Lazy-8 maneuver and a 25-second Immelmann turn maneuver are compared with those from full computational flight solutions that required days to complete. Finally, a cost-benefit assessment is included that demonstrates a compelling advantage for this approach.

\section{Nomenclature}

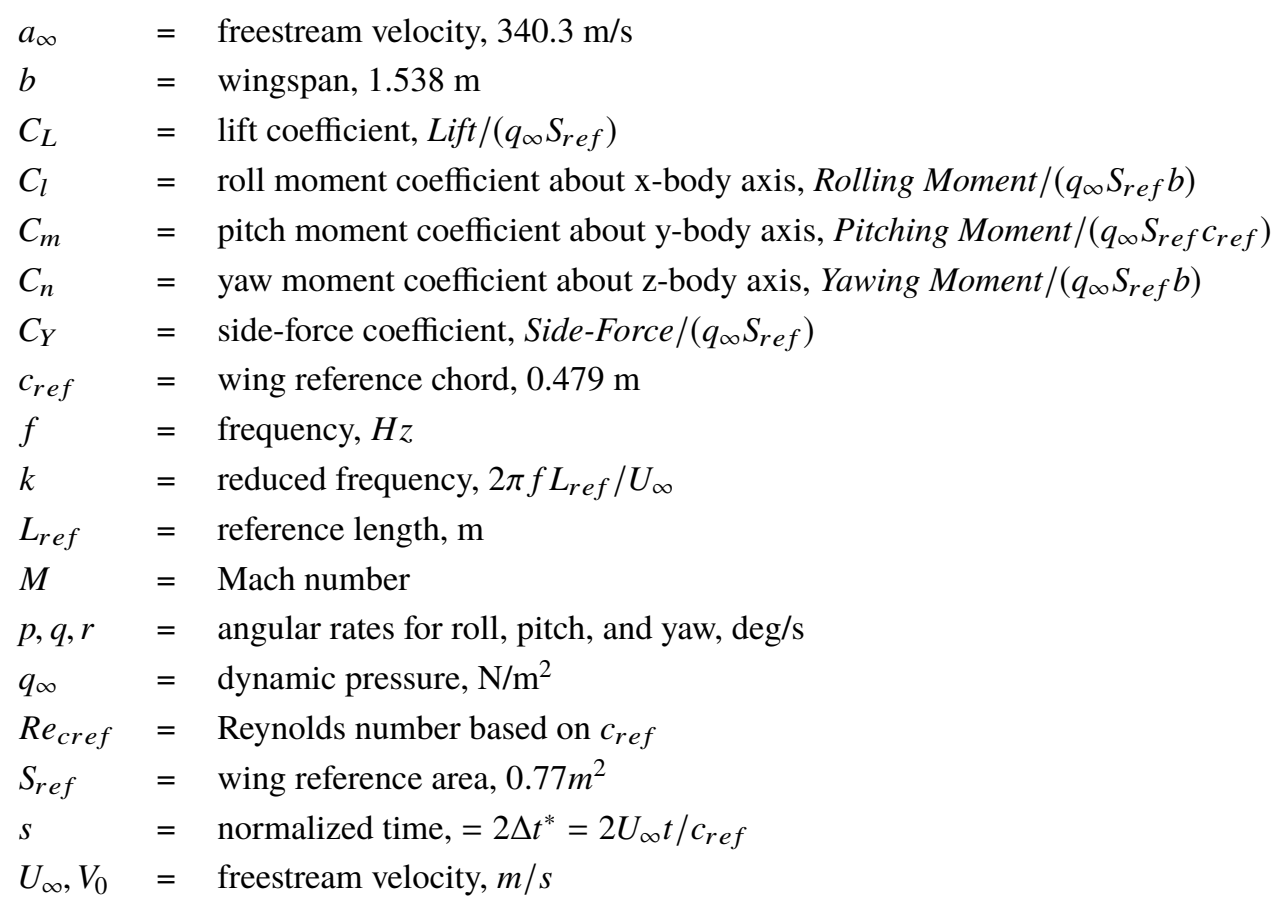

\footnotetext{
* Senior Research Engineer, Configuration Aerodynamics Branch, AIAA Associate Fellow

${ }^{\dagger}$ Research Student Trainee (PhD Candidate, Georgia Tech), Configuration Aerodynamics Branch, AIAA Student Member

${ }^{\dagger}$ Senior Research Engineer, Dynamic Systems and Control Branch, AIAA Associate Fellow

${ }^{\S}$ Senior Research Engineer, Flight Dynamics Branch, AIAA Senior Member

"IISenior Research Engineer, Flight Dynamics Branch, AIAA Associate Fellow
} 


$\begin{aligned} t, \Delta t & =\text { physical time and time-step, sec } \\ \Delta t^{*} & =\text { characteristic time step, } \Delta t U_{\infty} / c_{\text {ref }} \\ n_{\text {sub }} & =\text { number of subiterations } \\ y^{+} & =\text {inner scaling for wall-normal direction } \\ \alpha & =\text { angle of attack, deg } \\ \beta & =\text { angle of sideslip, deg } \\ \phi, \theta, \psi & =\text { Euler angles for roll, pitch, yaw in body axis system, deg }\end{aligned}$

\section{Introduction}

NASA has conducted research under the Technologies for Airplane State Awareness (TASA), a subproject of the Airspace Operations and Safety Program, to improve the stall models for civil transports in motion-based flight simulators [1-4]. These efforts have been in support of a pending Federal Aviation Administration (FAA) requirement [5] that enhanced flight simulator models be implemented by early 2019 for training pilots to recognize precursors to stall and to learn upset recovery strategies. While the majority of this research has relied on experimental data sources, a portion has been dedicated to exploring ways to infuse computational aerodynamic data into the stall models [6]. High fidelity computational fluid dynamics (CFD) tools offer the potential to approximate increments for ground-to-flight scaling effects, as well as to augment the dynamic damping derivative data for the simulators. The status of research in computing ground-to-flight scaling increments and dynamic derivatives for a generic T-tail (GTT) commuter jet is reported in Refs. [7, 8]. The search for more efficient techniques to provide computational data for computing dynamic damping derivatives is addressed in this paper.

For practical reasons, the aerodynamic coefficients for flight simulation databases are generally expressed in series expansions, thus creating the commonly used stability and control (S\&C) derivatives. In wind tunnels, conventional practice to obtain damping derivatives is accomplished by measuring the in-phase and out-of-phase responses from sinusoidal forced oscillations (FO) on wind-tunnel models. Initial attempts to include CFD data involved mimicking the classic FO approach with CFD tools [9-11]. A key advantage of the computational approach is that the limitation of very low speeds and hence Reynolds numbers due to wind-tunnel model loads is not an issue. While the computational FO approach has been reasonably successful, it is largely impractical for populating a database due to the long computational run times for each data point. More efficient approaches are needed before CFD will become a cost-effective means to augment flight simulator databases.

Since CFD is not constrained by model loads or structural interferences common to wind tunnels, an aircraft can be "flown" through maneuvers that are not physically possible using experimental techniques across the full flight Reynolds and Mach number range. Research has been underway for several years to develop unsteady aerodynamic reduced-order models (ROM) constructed from high-fidelity CFD simulations of specialty maneuvers that capture a broad frequency content and nonlinear flow regimes. These flight ROMs seek to match the predictive capability of full CFD simulations with computational times reduced from many hours to minutes.

Considerable progress has been made by researchers at the United States Air Force Seek Eagle Office (AFSEO). Morton and his colleagues [12, 13] have developed techniques for flying an aircraft through training maneuvers designed to excite the relevant flow physics that will be encountered during actual missions in all three axes (roll, pitch, and yaw). These simulations are termed "training maneuvers" because they are used to regress or "train" the mathematical models. Such maneuver simulations consist of translations and rotations that are often not physically possible to achieve by an actual aircraft in flight or during a wind-tunnel test. Next, system identification (SysID) techniques are applied to create a mathematical model of the aircraft response. Then, the model is tested against available experimental data and CFD simulations, previously unused in the model generation process, to verify its validity. If the model is unsatisfactory, then the process is repeated with additional training maneuvers. This approach was developed by the Department of Defense (DoD) for fixed wing combat aircraft where a classic derivative-based model breaks down when undergoing an agile maneuver at high angles of attack.

Another reduced-order modeling approach has been developed by Ghoreyshi and his colleagues [14] at the United States Air Force Academy (USAFA) that is a surrogate-based indicial theory approach. Here the indicial responses of the aerodynamic coefficients are computed directly with CFD through a series of step inputs for angle of attack, $\alpha$, sideslip, $\beta$, and roll, pitch, and yaw angular rates, $p, q$, and $r$, respectively, that capture their transients as they settle to a steady state. The total number of required CFD calculations for model generation is reduced by constructing a time-dependent surrogate model where the indicial responses are only computed at strategic points of $\alpha$ and Mach number, $M$, within the flight envelope. The indicial responses of the aerodynamic coefficients are integrated through 
time along the aircraft maneuver while simultaneously interpolating across $\alpha$ and $M$, within the flight envelope using a technique such as kriging. Hence the required number of indicial solutions depends on the level of granularity required to resolve the nonlinearities of the flight envelope. In practice, a surprisingly small number of solutions are required to generate a reasonable nonlinear model. In many cases, the computational cost of creating the ROM is comparable to "flying the CFD" through one full flight maneuver. Furthermore, once the ROM has been generated, it can be flown within seconds through any maneuver within the flight envelope. A primary limitation is the requirement for asymptotic convergence of the indicial response, which presents challenges with stalled flows.

The overall goal for the present work is to investigate the utility of the surrogate-based indicial response ROM approach as a potential candidate for efficiently augmenting the S\&C database for civil transport flight simulators. The first step and topic of this paper is to implement and verify the Ghoreyshi ROM methodology using two NASA flow solvers, USM3D and FUN3D. Prior to assessing the approach, a grid refinement study is conducted for an unmanned combat air vehicle (UCAV) configuration, and dynamic solution strategies are established for both flow solvers. ROM solutions are then presented to assess the predictive capability for classic forced-oscillating motions at low-angles of attack and various reduced frequencies. Then, the benefits and challenges of the ROM will be assessed through predictions of the half Lazy-8 and Immelmann turn maneuvers published in Ref. [14] that exhibit weakly nonlinear aerodynamics.

This paper lays a foundation for additional work that is needed before the goal of efficiently augmenting civil transport S\&C databases is achieved. The next step would be to confirm that the new ROM can predict, when present, any unsteady frequency dependence in dynamic S\&C derivatives for aircraft undergoing classic sinusoidal and wide-band oscillating motions. And ultimately, an approach must be developed to modify the unsteady indicial responses encountered during stall in an attempt to capture the overall lag and sluggesh behavior generally experienced during a stall.

\section{Geometry}

The Stability and Control Configuration (SACCON) geometry, depicted in Fig. 1 and described in detail in Ref. [15], was created by the North Atlantic Treaty Organization (NATO) Science and Technology Organization (STO) AVT-161 Task Group titled "Assessment of Stability and Control Prediction Methods for NATO Air \& Sea Vehicles" [16], and subsequently used in the follow-on AVT-201 Task Group "Extended Assessment of Reliable Stability \& Control Predictions Methods for NATO Vehicles" [17]. It is a generic representation of a UCAV configuration with a 53-deg swept leading edge that can be tested either sharp or rounded. The configuration of interest to this study is designated the 'SACCON-01 round-leading-edge' configuration, which has a sharp inboard leading edge segment that transitions to a medium-round leading edge on the outer wing panel. The outer wing panels are twisted about the leading edge to yield a 5-deg washout. The wingspan is $1.54 \mathrm{~m}$, root chord is approximately $1 \mathrm{~m}$, and reference chord, $c_{r e f}$ is $0.48 \mathrm{~m}$. The reference area, $S_{r e f}$, is $0.77 \mathrm{~m}^{2}$. The Point of Rotation (PoR) for pitch and yaw oscillations is positioned near the aft end of the root chord, downstream of the aerodynamic Moment Reference Point (MRP). The wind-tunnel model was designed to be mounted by a post sting attached at the PoR for tests in the German-Dutch Wind Tunnel (DNW-NWB) located in Braunschweig, Germany or a rear sting mount for the NASA Langley 14- by 22-Foot (14x22) Subsonic Tunnel in Hampton, Virginia, United States.

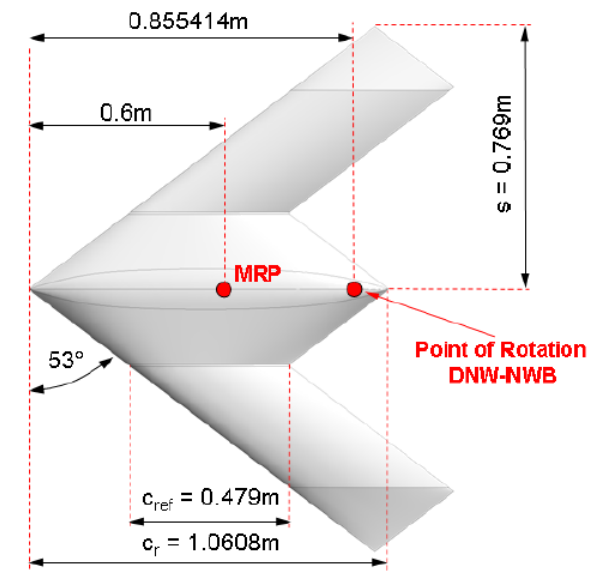

Fig. 1 Planform and geometric parameters of the SACCON configuration. 


\section{Computational Tools}

Two NASA flow solvers, USM3D and FUN3D, are used in this work. Both are exercised for time-accurate solution of the Reynolds-averaged Navier-Stokes (RANS) equations using the Spalart-Allmaras one-equation turbulence model.

\section{A. USM3D}

The legacy USM3D is a tetrahedral cell-centered RANS flow solver that has been in use for many years [18]. The present study uses a new developmental version of USM3D that is a parallelized, mixed-topology, cell-centered, finite volume Navier-Stokes flow solver [19]. Inviscid flux quantities are computed across each cell face using various upwind schemes. To improve the convergence and robustness of USM3D solutions, a hierarchical adaptive nonlinear iteration method (HANIM) has been recently implemented in the mixed-element USM3D [20]. Further enhancements to the new USM3D preconditioner and discretization scheme are reported in Refs. [21, 22]. Turbulence models currently available are the standard and negative variant [23] of the Spalart-Allmaras (SA) one equation model, the SA with Quadratic Constitutive Relation (QCR), and the two-equation k-epsilon turbulence model, the Menter Shear Stress Transport (SST) two-equation model. A general prescribed grid motion capability is available that can be advanced in time by a $2^{\text {nd }}$ - or $3^{r d}$-order time step scheme.

For the present study, the negative variant of the SA model was used for the USM3D computations. Inviscid fluxes are computed with Roe's flux difference scheme without limiting. The boundary conditions consist of a surface no-slip constraint on the wing and characteristic inflow/outflow on the outer box. Time is advanced with an implicit $2^{\text {nd }}$-order dual time-step scheme using exponential ramping either within each time step or over the global time steps. When ramping locally within each time step over 5,10 , or 15 subiterations, the Courant-Friedrichs-Lewy (CFL) number exponentially increases to a maximum of $148 ; 22,036$; and $3.27 \times 10^{6}$, respectively, to advance the meanflow and turbulence equations. With 5 subiterations, the low $\mathrm{CFL}_{\max }$ of 148 severely limits the subiterative convergence. Hence, with 5-subiteration solutions, a global exponential ramping is used that continues to increase and then holds a prescribed maximum CFL of $1 \times 10^{40}$.

\section{B. FUN3D}

FUN3D v13.3 is a parallelized, unstructured, node-based, finite-volume discretization, RANS flow solver [24]. Inviscid flux quantities are computed using a variety of upwind flux functions. Turbulence models currently available include SA, Menter k-omega SST, Wilcox k-omega, and detached eddy simulations, among others. Time accurate simulations may be advanced in time using up to $3^{r d}$-order time step schemes, where pseudotime advancement is dictated by a linear CFL-subiteration schedule within each iteration. An optimized $2^{\text {nd }}$-order backward differencing (BDF2opt) was utilized in the present work.

For the present study, the standard SA model was used for the FUN3D computations. Similar to the USM3D procedure, inviscid fluxes are computed with Roe's flux difference scheme without limiting. The boundary conditions consist of a surface no-slip condition on the wing and characteristic inflow/outflow on the outer box. Time is advanced with an implicit $2^{\text {nd }}$-order dual time-step scheme using a constant CFL number within each time step. For all of the conducted simulations, constant CFL values of 30 and 20 were used to advance the meanflow and turbulence equations, respectively. By using a constant CFL ramping, convergence for a given time step resolution is isolated to and controlled by the number of specified subiterations.

\section{Grid Motion}

A desired trajectory is initially defined by aerodynamic parameters $(\alpha, \beta, M)$ and Euler angles $(\phi, \theta, \psi)$. The relations described in Appendix A transform this trajectory from the wind-to-body and body-to-horizontal axes system. For USM3D simulations, a resulting trajectory file provides a set of translations and rotations to move the grid relative to the horizontal or inertial axes system. For FUN3D simulations, a general prescribed rigid body motion capability is available that relies on a series of $4 \times 4$ transformation matrices, which maps the moving body coordinates to the fixed initial reference frame at user-specified points in time [25]. Appendix A illustrates the format of the user-defined motion input files required for simulating rigid body maneuvers using the USM3D and FUN3D flow solvers.

\section{Grid Generation}

Computational grids were created using Heldenmesh ${ }^{\mathrm{TM}}$ v3.03, which is a multithread software system developed by the Helden Aerospace Corporation for the rapid generation of high quality, three-dimensional, mixed-element 
unstructured meshes about arbitrary complex configurations [26]. This capability was developed to streamline the application of CFD in a demanding production environment by transforming the mesh generation process from one currently measured in days-per-mesh to one measured in meshes-per-day. The Heldenmesh ${ }^{\mathrm{TM}}$ software system currently consists of two primary tools, Heldenpatch ${ }^{\mathrm{TM}}$ and Heldenmesh ${ }^{\mathrm{TM}}$. The former is the interface between the underlying Initial Graphics Exchange Specification (IGES) geometry definition and the Heldenmesh ${ }^{\mathrm{TM}}$ grid generator, and includes geometry clean-up, management, and geometric transformation capabilities. The Heldenpatch ${ }^{\mathrm{TM}}$ will typically provide around 95-percent of the surface patching automatically, leaving only a small amount of handwork to complete. An input-based spatial sourcing file will typically produce adequate spatial control of the cell size and distribution using only a few parameters with no additional user intervention needed. Heldenmesh ${ }^{\mathrm{TM}}$ was designed for compatibility with the NASA Tetrahedral Unstructured Software System (TetrUSS) [27, 28] in general and the VGRID mesh generation process in particular [29]. An important component of this system is the TetrUSS GridTool application, which is an interactive program for geometry processing. The GridTool application is currently utilized for interactive geometry processing in the Heldenmesh ${ }^{\mathrm{TM}}$ software system. A new integrated graphic user interface is planned for future releases.

A "family" of hybrid unstructured grids were generated on the clean SACCON wing that are suitable for the USM3D cell-centered and FUN3D node-based flow solvers. Characteristics of the grids are detailed in Table 1 where the global refinement factor, REFINE_FAC, of Heldenmesh ${ }^{\mathrm{TM}}$ was manually adjusted to produce nominally 5-, 10-, and 20-million total cells for USM3D and total nodes for FUN3D. The respective grid names are suffixed by "c" and "n" in Table 1

A near-wall first-node spacing was prescribed, based on flat-plate turbulent boundary layer theory, to achieve an approximate turbulent wall coordinate $\left(y^{+}\right)$of 0.5 at a longitudinal distance of $0.5^{*} c_{r e f}$ for a $R e_{c r e f}=1.57$ million. Since the near-wall layers are comprised of prismatic cells and the cell centroid height is positioned at one-half that of the first node of the grid, the initial spacing of the first node, $\delta_{i}$, was adjusted for USM3D to be twice that for FUN3D to yield a consistent $y^{+}$. Beyond the first layer of prisms, the layers grow into the field with stretching factors of $r_{1}=0.15$ and $r_{2}=0.02$, where the nodal spacing layers are defined by Eq. 1

$$
\delta_{i+1}=\delta_{i} \cdot\left[1+r_{1} \cdot\left(1+r_{2}\right)^{i}\right]^{i}
$$

The surface and volume discretization for the "family" of USM3D and FUN3D grids are shown in Figs. 2 and 3 . respectively. With REFINE_FAC $=1$, the maximum surface spacing was set to $6 \times 10^{-3} \mathrm{~m}$ and the minimum to $1 \times 10^{-4} \mathrm{~m}$. This combination can enable as high as 60-to-1 stretching along the leading edge with the smallest spacings clustered in the circumferential direction. These maximum and minimum surface spacings are scaled for each grid by the REFINE_FAC parameter in Table 1] The flat trailing-edge spacing is not scaled and is set to $2.345 \times 10^{-4} \mathrm{~m}$ to allow four points across the $9.4 \times 10^{-4} \mathrm{~m}$ thick surface.

Table 1 Characteristics of cell- and node-based "family" of grids. (Mc is million cells, Mn is million nodes)

\begin{tabular}{ccccccccc}
\hline \hline Grid & REFINE_FAC & $\begin{array}{c}\delta_{i} \\
{[\mathrm{~m}]}\end{array}$ & $\begin{array}{c}\text { Surf Tri } \\
{[\text { millions }]}\end{array}$ & $\begin{array}{c}\text { Nodes } \\
{[\text { millions }]}\end{array}$ & $\begin{array}{c}\text { Pyramids } \\
{[\text { millions }]}\end{array}$ & $\begin{array}{c}\text { Prisms } \\
{[\text { millions }]}\end{array}$ & $\begin{array}{c}\text { Tets } \\
{[\text { millions }]}\end{array}$ & $\begin{array}{c}\text { Total Cells } \\
{[\text { millions }]}\end{array}$ \\
\hline $5 \mathrm{Mc}$ & 1.718 & $5.75 \times 10^{-6}$ & 0.104 & 1.81 & 0.019 & 2.82 & 2.17 & $\mathbf{5 . 0 1}$ \\
$10 \mathrm{Mc}$ & 0.967 & $5.75 \times 10^{-6}$ & 0.236 & 3.86 & 0.033 & 6.36 & 3.62 & $\mathbf{1 0 . 0 1}$ \\
$20 \mathrm{Mc}$ & 0.593 & $5.75 \times 10^{-6}$ & 0.526 & 8.10 & 0.054 & 13.81 & 6.16 & $\mathbf{2 0 . 0 3}$ \\
\hline $5 \mathrm{Mn}$ & 0.846 & $2.875 \times 10^{-6}$ & 0.292 & $\mathbf{5 . 0 0}$ & 0.036 & 8.33 & 4.43 & 12.79 \\
$10 \mathrm{Mn}$ & 0.544 & $2.875 \times 10^{-6}$ & 0.611 & $\mathbf{1 0 . 0 1}$ & 0.059 & 17.22 & 7.21 & 24.49 \\
$20 \mathrm{Mn}$ & 0.360 & $2.875 \times 10^{-6}$ & 1.275 & $\mathbf{2 0 . 0 2}$ & 0.095 & 35.24 & 12.08 & 47.41 \\
\hline \hline
\end{tabular}

Setup for the initial grid required approximately 1-hour by an inexperienced user. Subsequent grids could be generated within minutes by changing the REFINE_FAC parameter and rerunning Heldenmesh ${ }^{\mathrm{TM}}$. The six grids were generated with Heldenmesh ${ }^{\mathrm{TM}}$ at an average rate of $90.1 \mathrm{~K}$ nodes or $229.1 \mathrm{~K}$ cells per second with a total computational time of 8.5 minutes on 32 threads of the NASA Langley Research Center (LaRC) K3-cluster of Sandy Bridge nodes, consisting of Intel Xeon E5-2670 processors. 

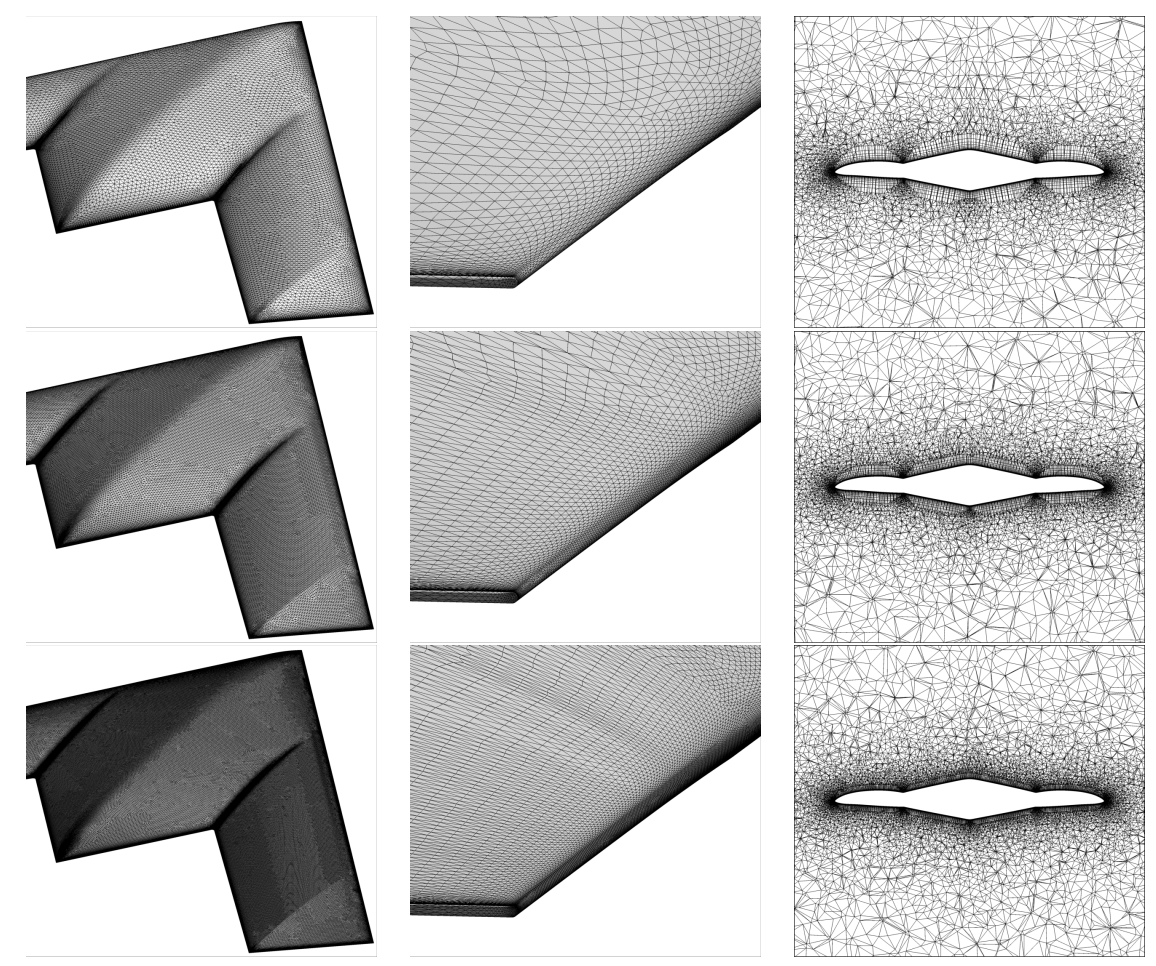

Fig. 2 USM3D cell-centered grids. From top to bottom: 5Mc, 10Mc, and 20Mc. From left to right: threequarter rear view of wing, closeup of leading edge at right wing tip, and slice through volume grid at $x=0.60 \mathrm{~m}$.
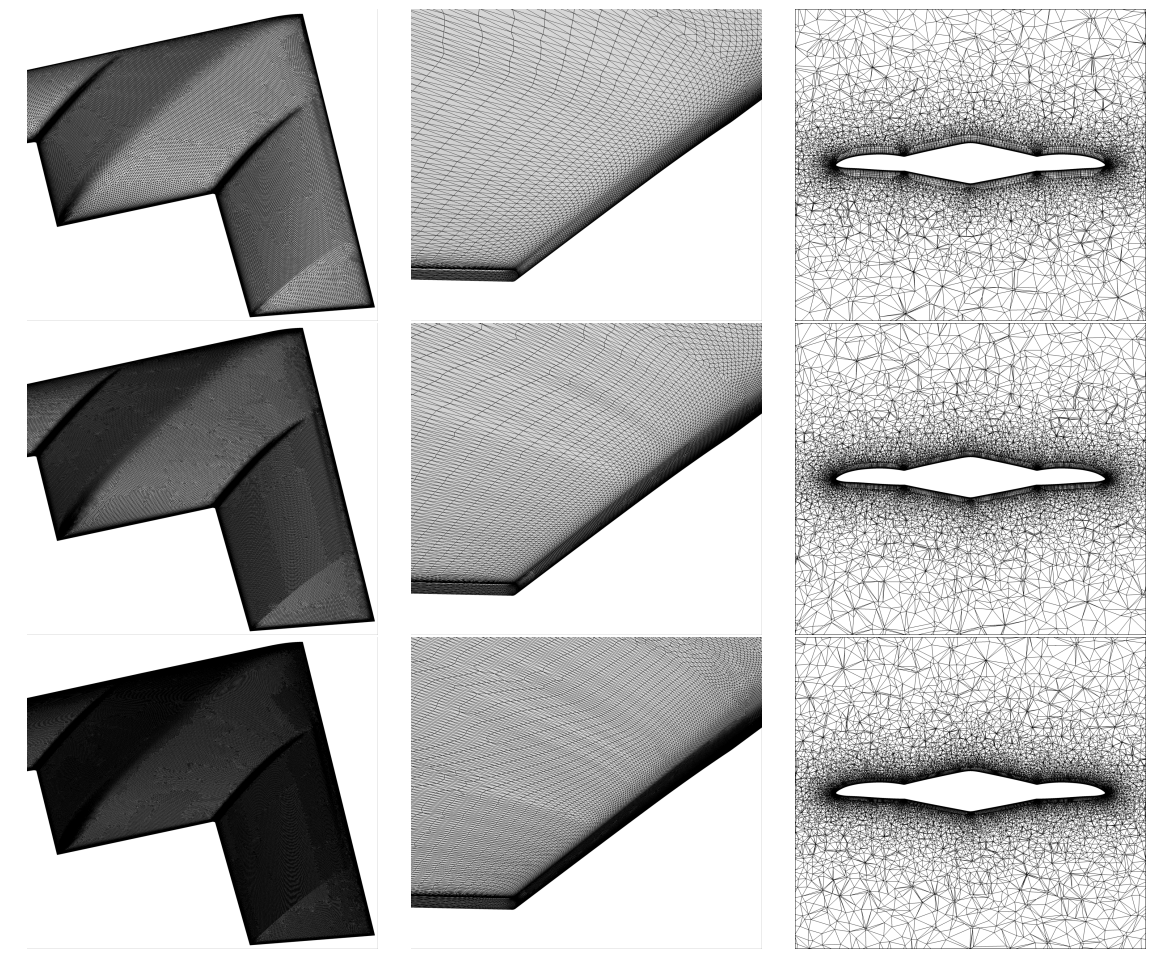

Fig. 3 FUN3D node-based grids. From top to bottom: 5Mn, 10Mn, and 20Mn. From left to right: threequarter rear view of wing, closeup of leading edge at right wing tip, and slice through volume grid at $\mathrm{x}=0.60 \mathrm{~m}$. 


\section{Reduced-Order Modeling}

\section{A. Aerodynamic Indicial Response Theory}

Initial applications of aerodynamic indicial response theory were first detailed by Tobak et al. [30, 31] and Reisenthal et al. [32, 33]. The unsteady aerodynamic response to longitudinal motions were modeled within linear flight regimes using indicial theory by accounting for contributions due to angle of attack and pitch rate, state parameters common to traditional stability-derivative models. Equation 2 expresses the unsteady lift and pitching moment, $C_{j}=\left[C_{L}, C_{m}\right]$, as a function of the convolution integrals with respect to any arbitrary time-varying maneuver defined by angle of attack, $\alpha$, and pitch rate, $q$. In Eq. 2. $C_{j_{0}}$ denotes the zero-angle-of-attack lift or pitching moment coefficients, provided by steady-state data. The terms $C_{j_{\alpha}}$ and $C_{j_{q}}$ represent the transient indicial responses of the system with respect to unit step changes in angle of attack and pitch rate, respectively. Direct identification of indicial responses have been difficult to measure experimentally due to the inability to create an instantaneous step motion. Historically, indicial responses were identified either analytically, for 2D configurations, or experimentally, in conjunction with system identification methods.

$$
C_{j}(t)=C_{j_{0}}+\frac{d}{d t}\left[\int_{0}^{t} C_{j_{\alpha}}(t-\tau) \alpha(\tau) d \tau\right]+\frac{d}{d t}\left[\int_{0}^{t} C_{j_{q}}(t-\tau) q(\tau) d \tau\right]
$$

More recently, Ghoreyshi and Cummings [34] leveraged the grid motion capabilities of modern CFD solvers to enable direct identification of indicial responses for maneuvering aircraft. The procedure assumes that the CFD solver allows specification of all translational and rotational degrees of freedom. These degrees of freedom are defined with respect to a reference point on the aircraft at each time step of the simulation. The initial aircraft velocity, $v_{0}$, is first calculated based on the initial angle of attack, sideslip angle, and Mach number. For successive iterations, the aircraft's reference point velocity, $v_{a}$, in the body-fixed inertial reference frame is calculated and specified to achieve the angle of attack, sideslip angle, and forward speed defining a prescribed maneuver. The translational degrees of freedom are defined by the relative velocity vector, $v_{a}-v_{0}$, of the aircraft's reference point. Rotation of the aircraft about the reference point is then defined using the roll, pitch, and yaw rotation angles. The ability to simulate such 6DOF maneuvers using CFD solvers allows for the simulation of both full-order solutions and indicial response functions.

Fig. 4 illustrates an example of the CFD-calculated approximations for the indicial response with respect to step changes in angle of attack and pitch rate. In this approach, a step change in angle of attack is achieved by specifying a grid motion, beginning at $t=0$, consisting of a translation downward and aft. During this motion, changes in the aerodynamic loads are isolated to contributions from angle of attack, while maintaining a zero-valued pitch rate. For a unit step change in pitch rate, the grid rotates at a constant, unit pitch rate. Because angle of attack changes with rotation, an additional grid motion is specified to translate upward and aft to maintain a local $\alpha=0^{\circ}$. This grid motion technique can also be applied to lateral motions to allow for the approximation of indicial responses with respect to sideslip angle, roll rate, and yaw rate. The benefit of this approach is that indicial responses for 2D or 3D configurations can be calculated at any flight condition, overcoming the identification problems inherent to analytical and experimental methods. Using this CFD-based identification approach, the linear aerodynamic indicial response model presented in Eq. 2 has been used to calculate the unsteady aerodynamics of longitudinal motions [34].

For nonlinear flows, the linear aerodynamic indicial response theory of Eq. 2 has been extended by use of parameterized indicial response functions [14, 35]. The nonlinear aerodynamic indicial response model for longitudinal coefficients is given by Eq. 3. Analogous to aerodynamic databases, flow nonlinearity may be captured by sampling a system's locally linear indicial response as a function of the flight space parameter values, where the degree of nonlinearity resolved scales with the sampling of indicial responses. In the case of longitudinal aerodynamics, the indicial response with respect to angle of attack is assumed to vary with angle of attack and Mach number, whereas for pitch rate, the indicial response is assumed to vary only with Mach number with negligible variations in angle of attack at low to moderate angles of attack. These parameterizations are consistent with those typical of aerodynamic databases.

$$
C_{j}(t)=C_{j_{0}}(M)+\frac{d}{d t}\left[\int_{0}^{t} C_{j_{\alpha}}(t-\tau, \alpha, M) \alpha(\tau) d \tau\right]+\frac{d}{d t}\left[\int_{0}^{t} C_{j_{q}}(t-\tau, M) q(\tau) d \tau\right]
$$

Ghoreyshi and Cummings [14, 35] later extended this formulation for the prediction of unsteady aerodynamic responses to lateral motions, enabling estimates for the aerodynamics for 6DOF maneuvering aircraft. Consistent with traditional aircraft flight dynamics assumptions, the lateral loads are assumed to only depend on sideslip angle $(\beta)$, normalized roll rate $(p)$, and normalized yaw rate $(r)$. The unsteady lateral aerodynamics, namely side force $(Y)$, rolling 

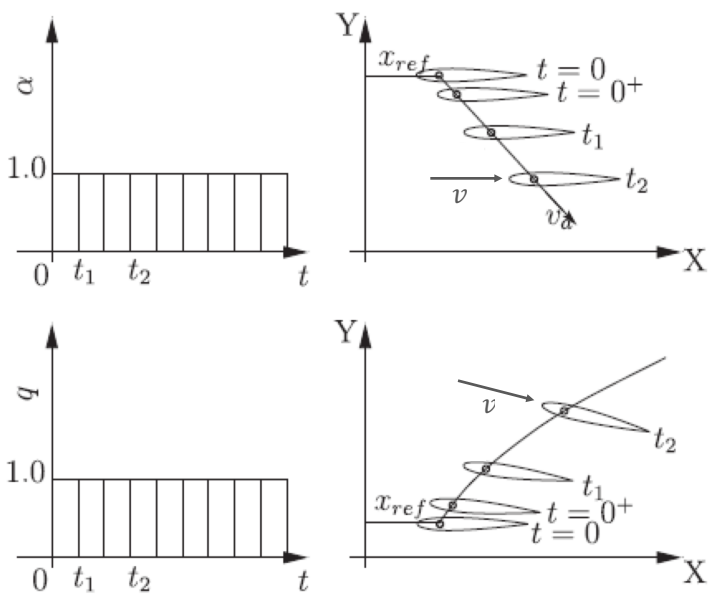

Fig. 4 Grid motion illustration of indicial responses from Ref. [14]. Upper: $\Delta \alpha=1^{\circ}, q=0 \mathrm{rad} / \mathrm{sec}$, Lower: $\Delta q=1 \mathrm{rad} / \mathrm{sec}, \alpha=0^{\circ}$.

moment $(l)$, and yawing moment $(n)$, may then be calculated using Eq. 4 The parameterization of the lateral/directional indicial responses, $C_{k}=\left[C_{Y}, C_{l}, C_{n}\right]$, are also consistent with those used in the longitudinal formulation.

$$
\begin{aligned}
C_{k}(t)=\frac{d}{d t}\left[\int_{0}^{t} C_{k_{\beta}}(t-\tau, \alpha, M) \beta(\tau) d \tau\right] & +\frac{d}{d t}\left[\int_{0}^{t} C_{k_{p}}(t-\tau, M) p(\tau) d \tau\right] \\
& +\frac{d}{d t}\left[\int_{0}^{t} C_{k_{r}}(t-\tau, M) r(\tau) d \tau\right]
\end{aligned}
$$

In order to calculate the nonlinear responses, $C_{j \alpha}(t, \alpha, M)$ for example, the flight maneuver space is first parameterized by a set of angle of attack samples, $\alpha=\left[\alpha_{1}, \alpha_{2}, \ldots, \alpha_{n}\right]$, and a set of Mach number samples, $M=\left[M_{1}, M_{2}, \ldots, M_{n}\right]$. The responses are then calculated at each angle of attack, $\alpha_{i}(i=1,2, \ldots, n)$, for each Mach number sample, $M_{i}$, by first converging a static CFD simulation at the given angle of attack and Mach number and then performing a dynamic CFD simulation, for which grid motion capabilities are used to perform a unit step in angle of attack to $\alpha=\alpha_{i}+1$. The response function is then computed from the CFD solution by taking the difference between the time-varying response due to the unit step change and the static initial solution at $\alpha=\alpha_{i}$. This process is repeated for each unique combination of flight space sampling for a total number of indicial response simulations equal to $\alpha_{i} \times M_{i}$ for the nonlinear response $C_{j_{\alpha}}(t, \alpha, M)$. These same simulation requirements apply to the nonlinear responses with respect to sideslip angle, $C_{k_{\beta}}(t, \alpha, M)$; where instead, a unit step from $\beta=0^{\circ}$ is simulated at each unique combination of angle of attack and Mach number. The nonlinear responses with respect to the angular rates, $C_{j_{q}}(t, M), C_{k_{p}}(t, M)$, and $C_{k_{r}}(t, M)$, only depend on Mach number, where unit steps in each angular rate are simulated at $\alpha=0^{\circ}$ for each Mach number sample, $M_{i}$. The utility of the nonlinear aerodynamic indicial response theory presented in Eqs. 3 and 4 is assessed in the present work as a viable means of populating S\&C databases for civil transport flight simulators.

The sampling of the flight space may be completed using a variety of sampling strategies, including the methods of full-factorial design, Latin hypercube sampling, or sampling based on optimality criteria as reviewed in Ref. [36]. The maximum sampled values for angle of attack and Mach number should be chosen to define the limits of the flight space corresponding to the flight maneuvers to be evaluated by the reduced-order models. In the present work, only positive values of angle of attack are used with the underlying assumption that the response is symmetric between positive and negative angles of attack. The density of sampling should be carefully selected to balance the computational cost vs. the degree of resolution needed to capture the salient nonlinearities throughout the maneuvers to be predicted.

In the present work, a uniform sampling of one degree angle of attack is used with ranges of $\alpha=\left[0^{\circ}-10^{\circ}\right]$, and Mach numbers are sampled at $M=[0.05,0.15,0.3]$ to provide a sampling corresponding to the bounds of the prescribed maneuver space. A kriging surrogate modeling approach, first demonstrated in Ref. [14], is used to interpolate the database of simulated indicial responses to better resolve the flow nonlinearities encountered by any arbitrary prescribed flight maneuver. From this, the granularity of the database can be assessed by selective use of indicial responses from within the database. 


\section{B. Kriging Surrogate Modeling}

Surrogate modeling based on kriging interpolation has found widespread use in flight dynamics and aeroelastic studies as a means of reducing the computational costs associated with model identification [14, 37]. Kriging modeling is implemented in the present work using the MATLAB Design and Analysis of Computer Experiments (DACE) toolbox [38].

Following the formulation implemented in Ref. [14], the indicial responses are viewed as a set of time-correlated spatial processes, where the output is a simulated or 'observed' transient response. Universal-type kriging surrogate models may be used to estimate indicial responses, corresponding to the flight conditions at a given point along a maneuver, without the need for direct simulation of the response. A unique surrogate model is created for each discrete time step of the indicial response for a total of $n$ surrogate models as a function of the flight maneuver space inputs, i.e., one predicted response for each point along the maneuver, as created from $n$ surrogate models.

The modeling process begins with the definition of an input matrix, $\mathbf{X}(m \times d)$ in Eq. 5, and a corresponding output matrix of observed responses, $\mathbf{Y}(m \times n)$ in Eq. 6. The columns of the input matrix represent the number, $d$, of independent flight space variables, $x$, whereas the rows of the input matrix represent the number of samples, $m$, or unique combinations of values for the input variables. In modeling indicial responses with respect to angle of attack using the current sampling strategy, there are $d=2$ independent variables, $\alpha$ and $M$, corresponding to indicial responses sampled at $m=33\left(\alpha_{n} \times M_{n}\right)$ unique locations in the flight maneuver space. The rows of the output matrix also correspond to the number of samples, however, the columns of the output matrix now correspond to the $n$ discrete time steps or iterations of the numerically simulated indicial responses.

$$
\begin{aligned}
& \mathbf{X}=\left[\begin{array}{cccc}
x_{11} & x_{12} & \ldots & x_{1 d} \\
x_{21} & x_{22} & \ldots & x_{2 d} \\
\vdots & \vdots & \vdots & \vdots \\
x_{m 1} & x_{m 2} & \ldots & x_{m d}
\end{array}\right] \\
& \mathbf{Y}=\left[\begin{array}{cccc}
y_{11} & y_{12} & \ldots & y_{1 n} \\
y_{21} & y_{22} & \ldots & y_{2 n} \\
\vdots & \vdots & \vdots & \vdots \\
y_{m 1} & y_{m 2} & \ldots & y_{m n}
\end{array}\right]
\end{aligned}
$$

Universal kriging models the prediction at a given point in time, $\hat{y}_{i}$, at a new combination of values in the input variable space, $\mathbf{x}^{*}$, as the sum of a deterministic mean response value, $\mu_{i}$, and a zero-mean autocorrelated error, $\epsilon_{i}$, as given by Eq. 7

$$
\hat{y}_{i}\left(\mathbf{x}^{*}\right)=\mu_{i}\left(\mathbf{x}^{*}\right)+\epsilon_{i}
$$

The objective of kriging modeling is to minimize the mean squared error, $\phi$ in Eq. 8, of the modeling prediction $\hat{y}$ over the parameter space, where $E[\ldots]$ is the covariance of the quantity of interest [38]. For a linear regression of the parameter space, the resulting mathematical expressions for the mean response and autocorrelated error are given by Eqs. 9 and 10.

$$
\phi\left(\mathbf{x}^{*}\right)=E\left[\left(\hat{y}\left(\mathbf{x}^{*}\right)-y\left(\mathbf{x}^{*}\right)\right)^{2}\right]
$$

The mean response is written as a linear combination of regression functions, $f_{j}$, and their corresponding regression coefficients, $\beta_{i j}$, for the $j$-th regression function at time step $i$, for $i=1, \ldots, n$. In the present work, the regressions are defined as follows: $f_{0}\left(\mathbf{x}^{*}\right)=1, f_{1}\left(\mathbf{x}^{*}\right)=\alpha^{*}$, and $f_{2}\left(\mathbf{x}^{*}\right)=M^{*}$.

$$
\mu_{i}\left(\mathbf{x}^{*}\right)=\sum_{j=0}^{n} \beta_{i j} f_{j}\left(\mathbf{x}^{*}\right)
$$


The autocorrelated error is written in terms of $\mathbf{r}\left(\mathbf{x}^{*}\right)$, the correlation matrix between the sampled points and the new evaluation point, $\mathbf{R}$, the correlation function matrix for sampled points, $\mathbf{F}$, the regression functions at the sampling points, $\mathbf{Y}$, and $\beta$. Substituting the expressions for the mean response and autocorrelated error into Eq. 7)results in the final prediction expression given by Eq. 11 .

$$
\begin{gathered}
\epsilon_{i}\left(x^{*}\right)=\mathbf{r}^{T} \mathbf{R}^{-1}(\mathbf{Y}-\mathbf{F} \beta) \\
\hat{y}_{i}\left(\mathbf{x}^{*}\right)=\sum_{j=0}^{n} \beta_{i j} f_{j}\left(\mathbf{x}^{*}\right)+\mathbf{r}^{T} \mathbf{R}^{-1}\left(\mathbf{Y}_{i}-\mathbf{F} \beta\right)
\end{gathered}
$$

An illustration of the kriging surrogate modeling process for the prediction of a single indicial response (with respect to angle of attack) for a given point in the maneuver, where $\alpha=4.5^{\circ}$ and $M=0.25$, is shown in Fig. 5 . In this modeling procedure, $n$ surrogate models are generated, one for each point in discrete time. In consideration of the convolution process, an interpolated indicial response is needed at each point in time along the maneuver based on the current value of the flight space parameters. This process is utilized for the nonlinear indicial responses with respect to angle of attack and sideslip angle. A linear interpolation is used for the indicial responses with respect to the angular rates.

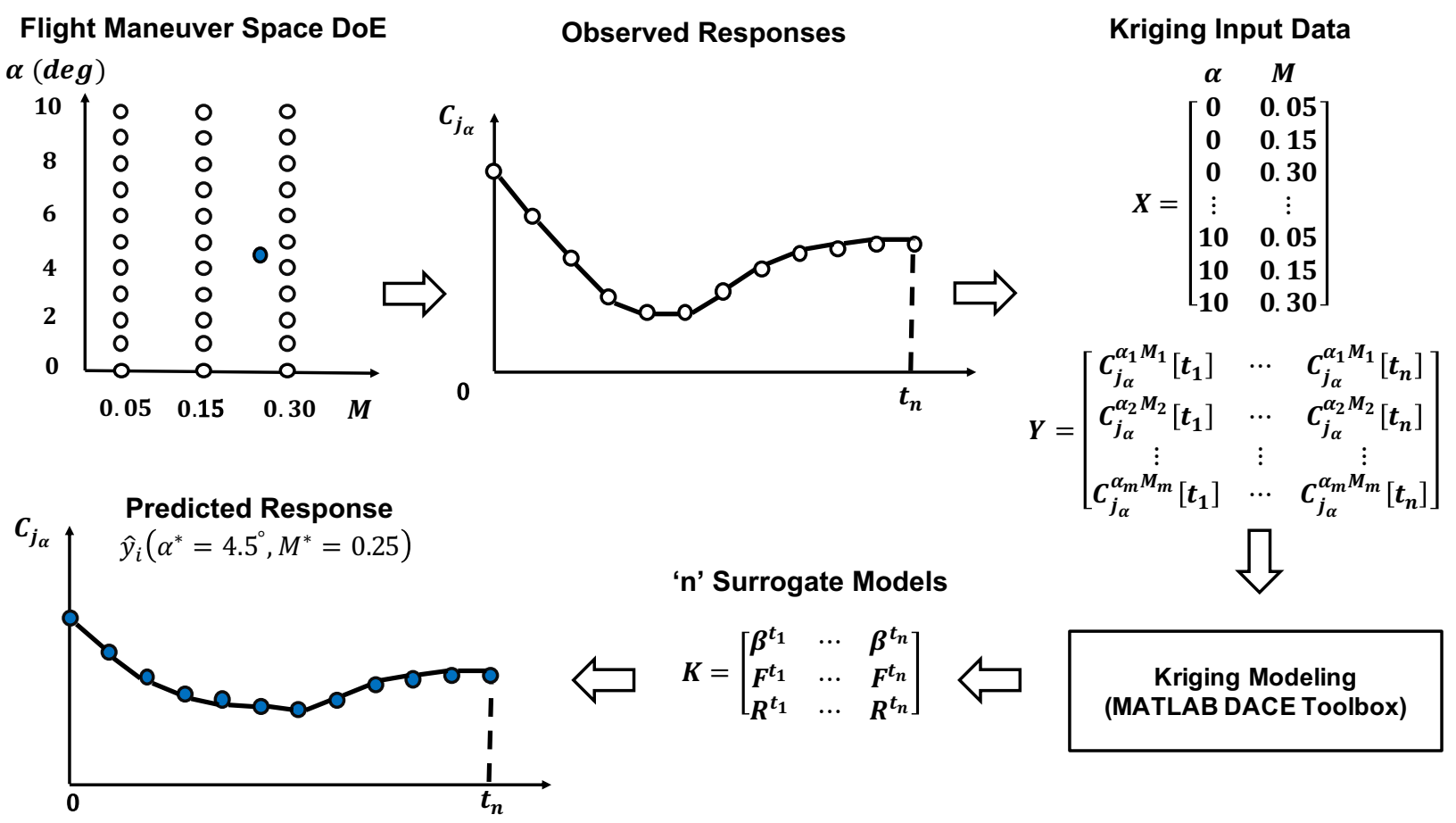

Fig. 5 Kriging modeling of indicial responses.

\section{Results \& Discussion}

The accuracy and efficiency of the indicial ROM will be evaluated through correlations with several full CFD flight maneuver solutions using SACCON. This section begins with a brief description of the evaluation flight maneuvers, followed by an assessment of CFD sensitivities to grid and time-step variations. Based on guidance from these results, the CFD indicial response solutions that serve as input to the ROM were computed. And finally, various ROM predictions are studied to provide application guidelines for future applications. 


\section{A. Description of Evaluation Flight Maneuvers}

\section{Half Lazy-8}

An aircraft performing a half Lazy-8 [14], illustrated in Fig. 6, enters and exits the maneuver from a straight and level condition. The angle of attack and sideslip angles are constrained within $\left[0^{\circ}\right.$ to $\left.10^{\circ}\right]$ and $\left[-4^{\circ}\right.$ to $\left.4^{\circ}\right]$, respectively, and Mach numbers within [0.1 to 0.28]. Figure 6(a) shows the aircraft making a $180^{\circ}$ turn. The aircraft starts a steep climb to reduce flight speed (as shown) in order to make the tight turn. The aircraft rolls left as the pitch angle decreases, such that the vehicle is at zero pitch and maximum roll angle at a $90^{\circ}$ yaw angle, as shown in Fig. 6(d) This is followed by a descent trajectory and decreasing roll angle, increasing pitch angle, and regaining speed until the vehicle reaches the initial velocity and altitude.

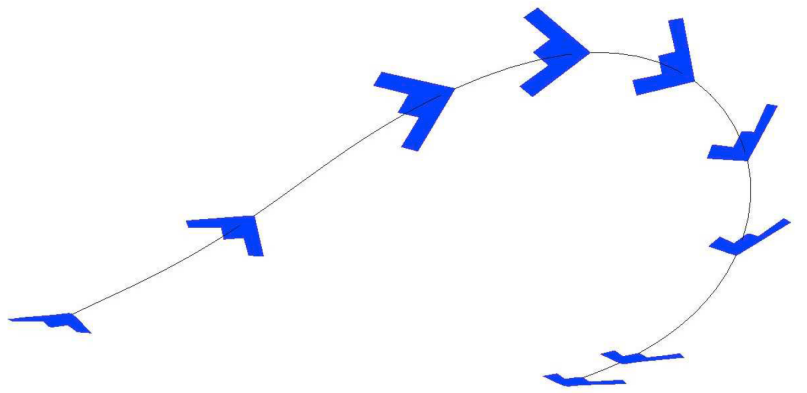

(a) Flight Trajectory

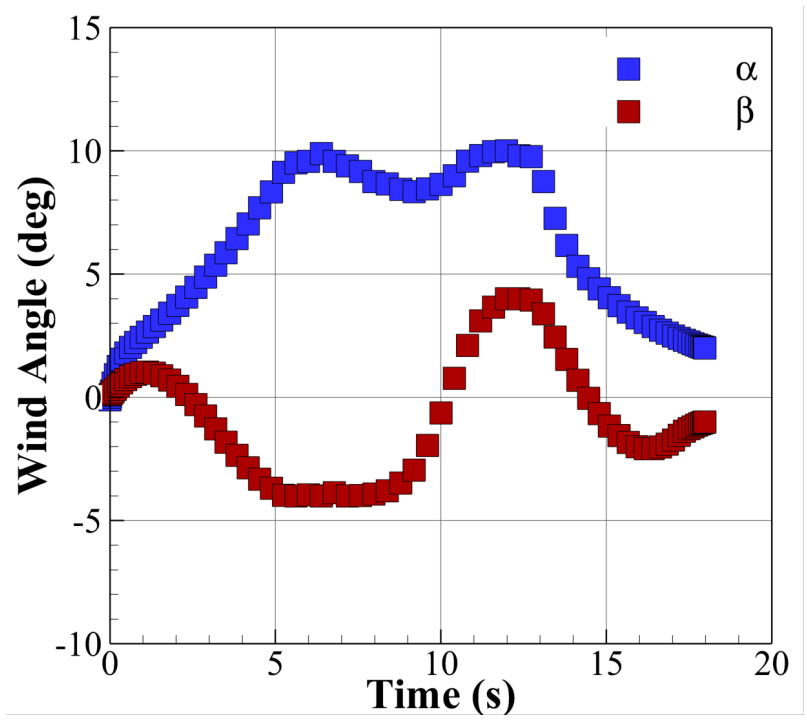

(c) Aerodynamic Angles

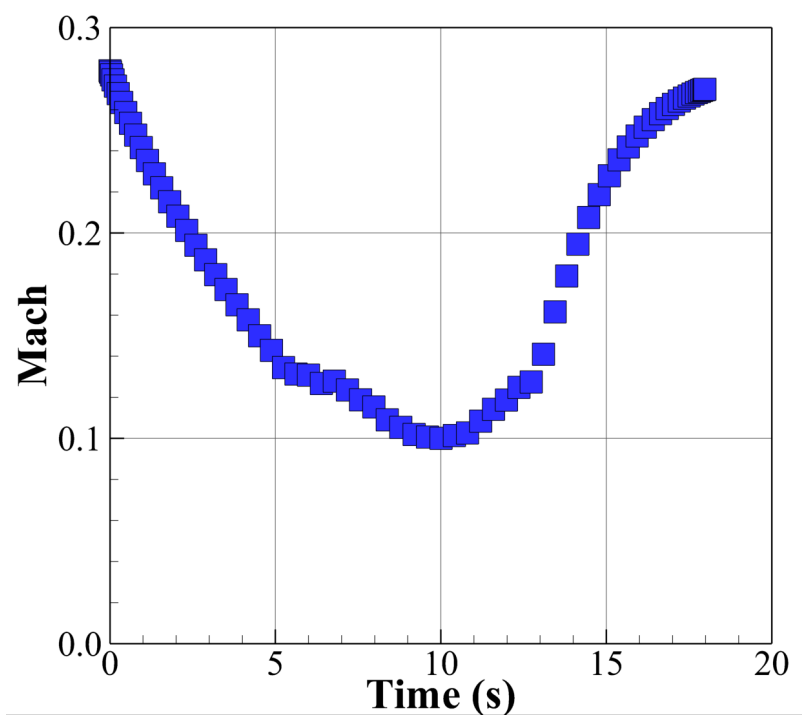

(b) Mach Number

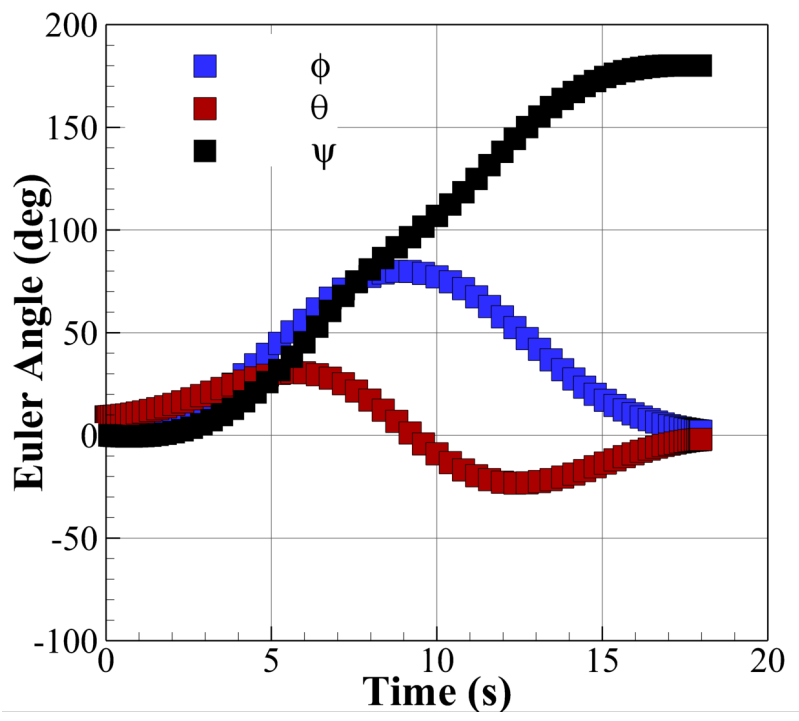

(d) Euler Angles

Fig. 6 Half Lazy-8 maneuver from Ref. [14]. 


\section{Immelmann turn}

The Immelmann turn [14], depicted in Fig. 7, is characterized by a half loop with a half roll at the end. The angles of attack and sideslip angles are constrained within $\left[-3^{\circ}\right.$ to $\left.10^{\circ}\right]$ and $\left[-4^{\circ}\right.$ to $\left.4^{\circ}\right]$, respectively, and Mach numbers within [0.07 to 0.28]. The maneuver starts with a steep climb and a resultant decrease in speed as shown in Fig. 7(b), At the maximum pitch angle, the aircraft changes heading from $0^{\circ}$ toward $180^{\circ}$. As the aircraft continues on its reverse heading, it performs a half roll to level the wing.

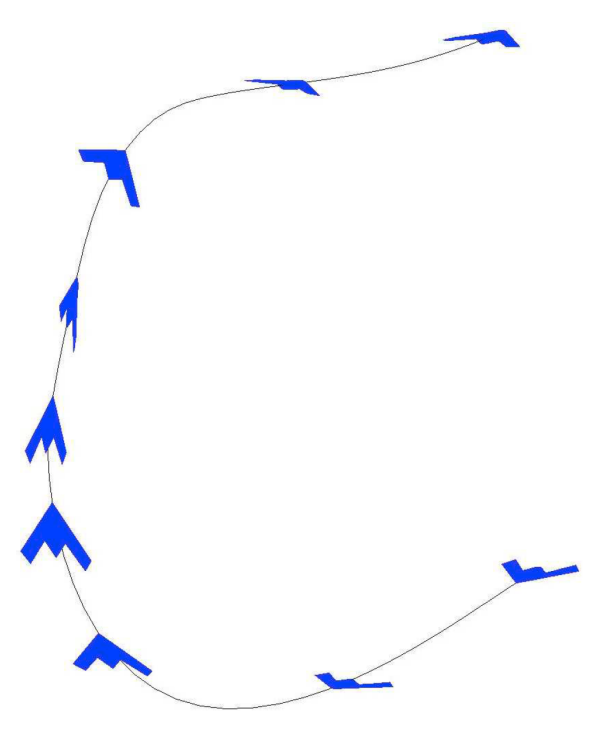

(a) Flight Trajectory

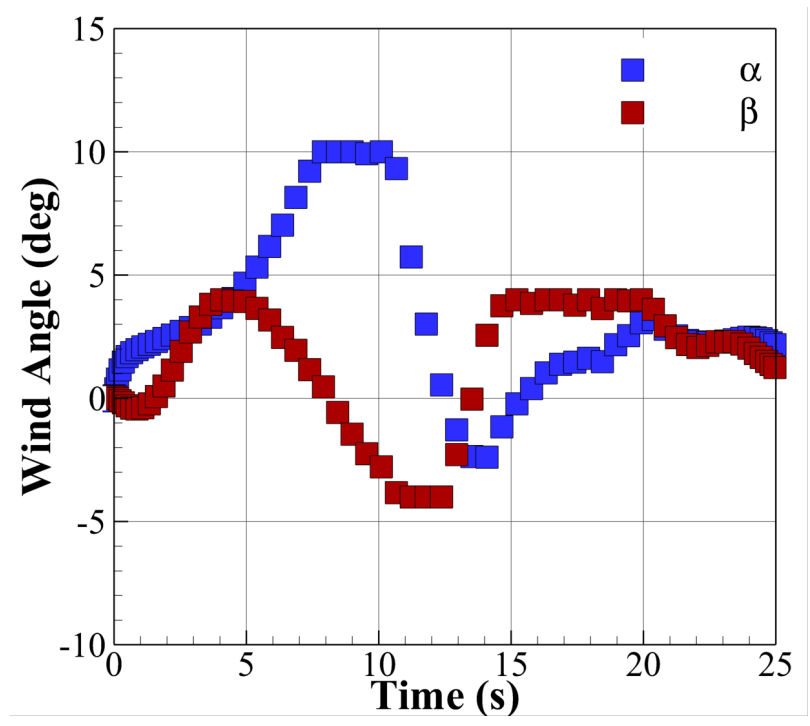

(c) Aerodynamic Angles

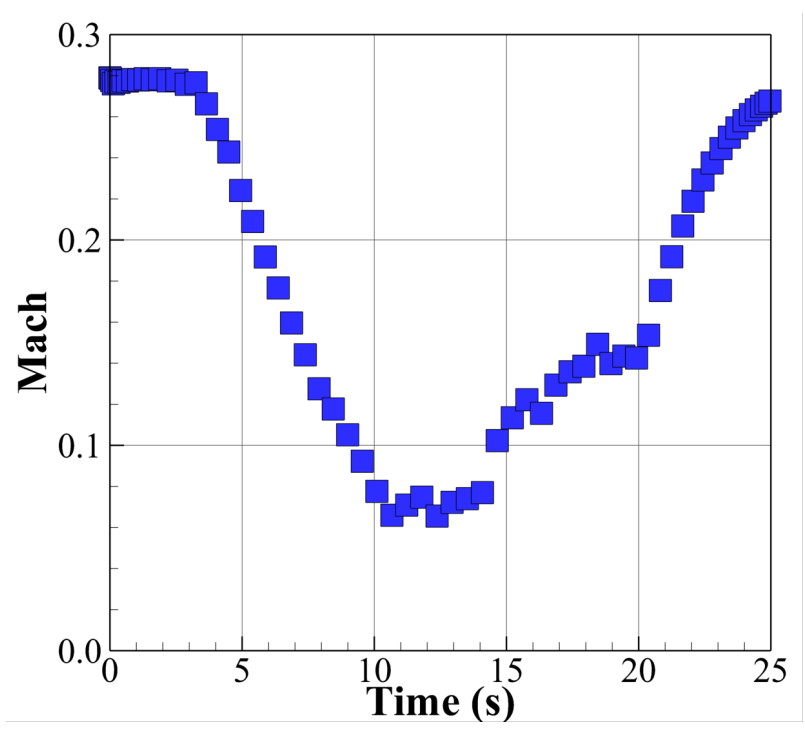

(b) Mach Number

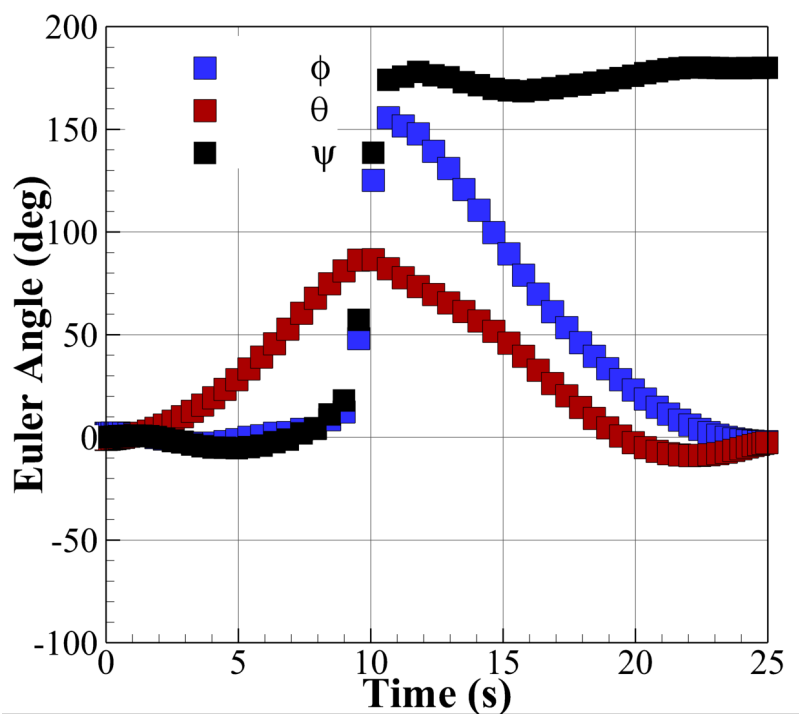

(d) Euler Angles

Fig. 7 Immelmann turn maneuver from Ref. [14]

\section{Sinusoidal forced oscillation}

The forced sinusoidal oscillation solutions are generated using nondeforming solid-body rotation of the full grid about the $\mathrm{x}, \mathrm{y}$, or $\mathrm{z}$ body axis and are initialized by restarting from a converged static solution at the prescribed angle of attack, $\alpha_{0}$, and Mach number, $M_{0}$. Two full sinusoidal oscillations are simulated to allow for initial transients to sufficiently decay. 


\section{B. Sensitivities to CFD Parameters}

\section{Effect of grid refinement on static angle-of-attack sweep}

A series of static time-accurate RANS flow solutions were generated at 21 angles of attack between $\left[0^{\circ}\right.$ to $\left.30^{\circ}\right]$ to assess the sensitivity of USM3D and FUN3D to the grid densities presented in Table 1 . The flow conditions are $M_{\infty}=0.15$ and $R e_{\text {cref }}=1.57$ million using the SA turbulence model. The time step was $\Delta t \approx 0.00094 \mathrm{~s}$ based on a prescribed characteristic time step of $\Delta t^{*}=0.10$, where $\Delta t=\Delta t^{*} \cdot c_{\text {ref }} / U_{\infty}$. A total of 1,000 time steps were simulated for $\alpha=\left[0^{\circ}\right.$ to $\left.8^{\circ}\right]$, and 1,500 steps for $\alpha=\left[9^{\circ}\right.$ to $\left.30^{\circ}\right]$. All solutions were confirmed to be well-converged.

Comparisons with lift and pitching moment data from the German-Dutch DNW-NWB wind tunnel are shown in Fig. 8 . The aerodynamics are characterized by a highly nonlinear behavior at the higher angles of attack, including a sharp pitch break around $\alpha=17^{\circ}$. The vortical flow physics producing these behaviors is well analyzed and interpreted in Ref. [39] and extensive CFD-to-experiment correlations with this data set are published in Refs. [40, 41]. The USM3D (open symbols) and FUN3D (solid symbols) results are in very close agreement with each other up to $\alpha=12^{\circ}$, but both significantly underpredict pitching moment. This observed underprediction of $C_{m}$ over the lower angle-of-attack range was typical for all eight flow solvers correlated with the data in Ref. [40]. The 5-, 10-, and 20-million element grids display some sensitivity in the $15^{\circ}$ to $20^{\circ}$ angle-of-attack range, and more so at the higher post-stall angles of attack. The cell-centered grid 20Mc captures the pitch break, which is caused by a sudden state change in leading-edge separation and subsequent vortex formation. The USM3D and FUN3D flow solvers both exhibit an expected level of uncertainty in the stall and post-stall region consistent with that from the eight flow solvers in Ref. [40].
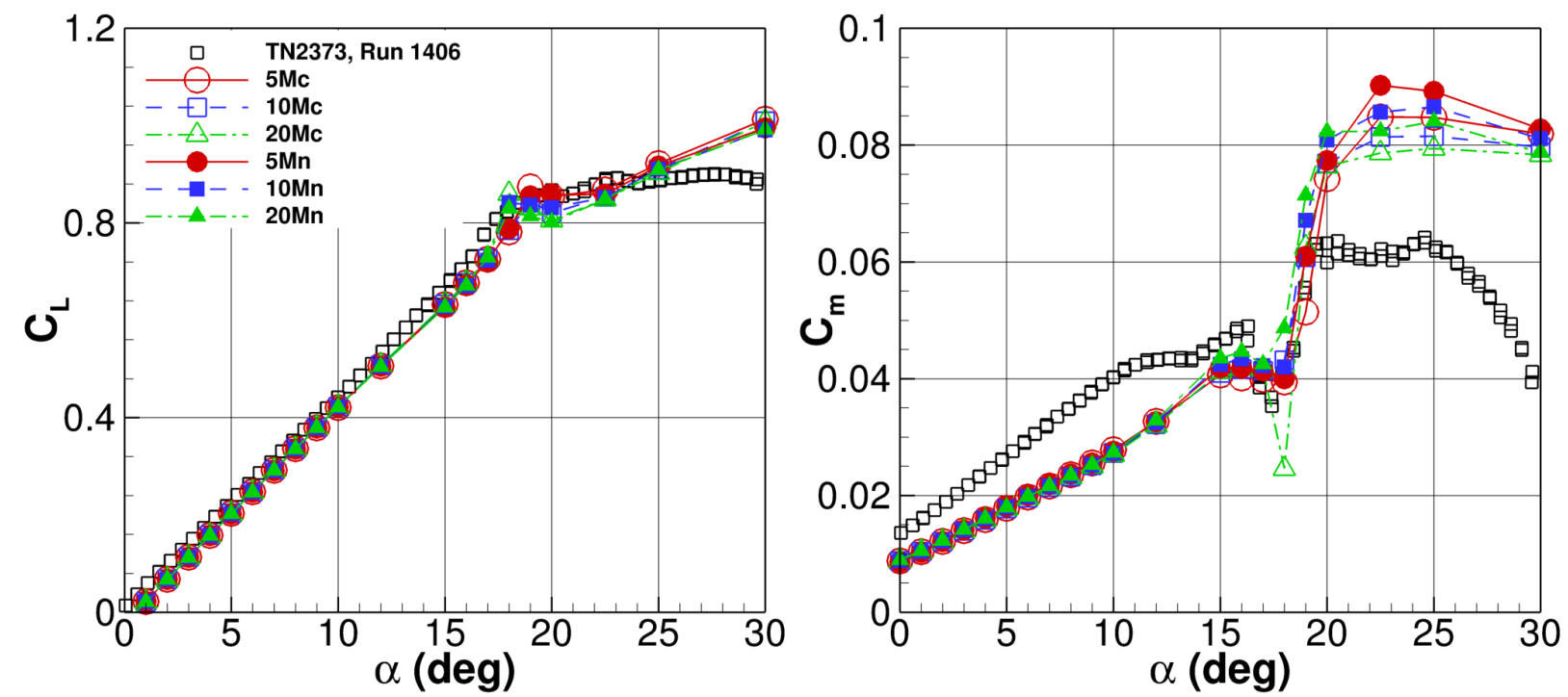

Fig. 8 Effect of SACCON grid refinement on static lift and pitching moment coefficient. Open symbols: USM3D/SA, solid symbols: FUN3D/SA. $M_{\infty}=0.15$ and $R e_{\text {cref }}=1.57 \times 10^{6}$.

\section{Effect of grid refinement and solution strategy on the half Lazy-8 maneuver}

Prior to choosing a grid for the indicial ROM assessment, a small study was performed using the SACCON half Lazy-8 maneuver from Ref. [14] to examine solution sensitivities to flow solver, grid density, subiterations, and time step size in a dynamic flight environment. From this, a selection of grid and solution strategy is made for generating the indicial response solutions and to potentially aid future studies.

A comparison of USM3D and FUN3D aerodynamic coefficients of lift, pitching moment, rolling moment, yawing moment, and side force is presented in Fig. 9 over the full 18-second half Lazy-8 maneuver depicted in Fig. 6. The solutions were generated on the 10-million element grids with a characteristic time step of $\Delta t^{*}=0.10$ and $n_{\text {sub }}=10$ subiterations. The two codes are typically in close agreement, with the largest differences occurring in the yawing moment coefficient, which is small in magnitude since the SACCON is a thin, tailless aircraft. Subsequent parameter sensitivity assessments focus on the longitudinal pitching moment, $C_{m}$, and directional yawing moment, $C_{n}$, coefficients. 


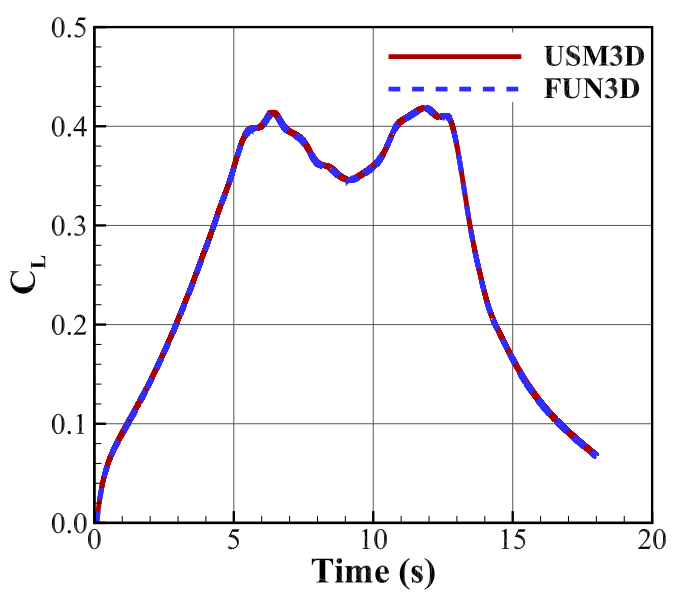

(a) Lift

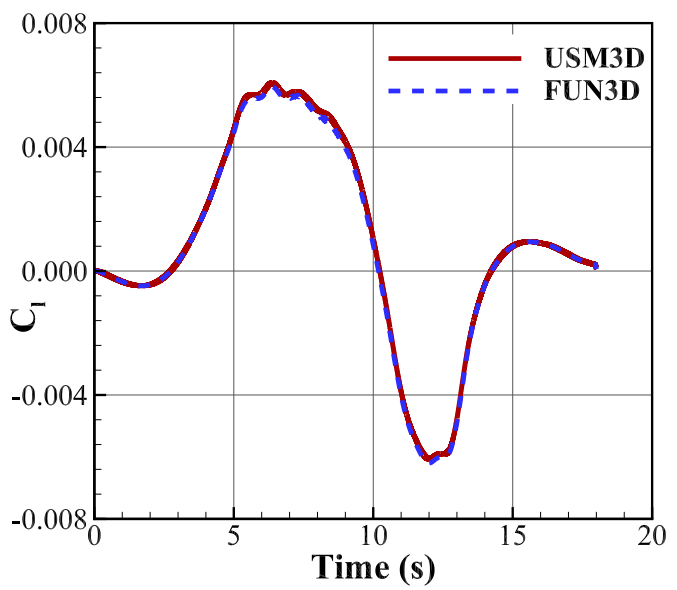

(c) Rolling Moment

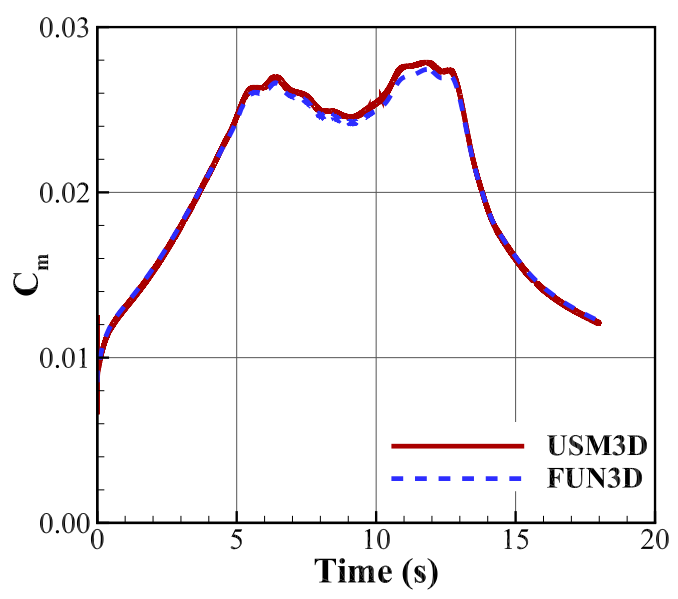

(b) Pitching Moment

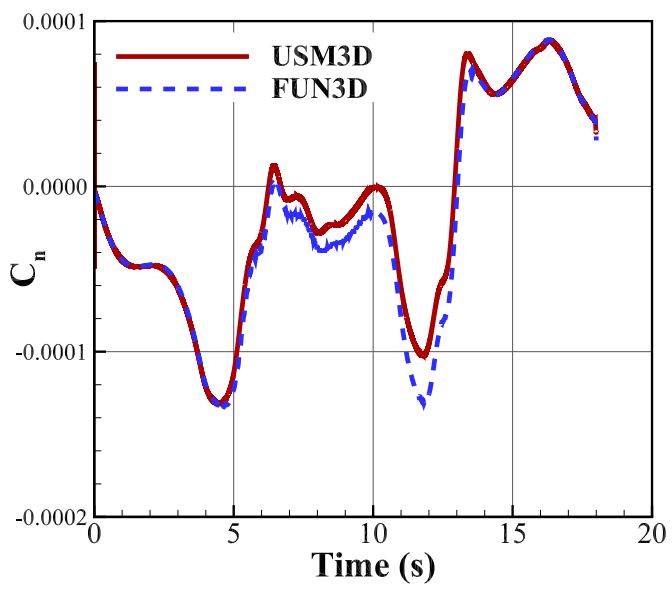

(d) Yawing Moment

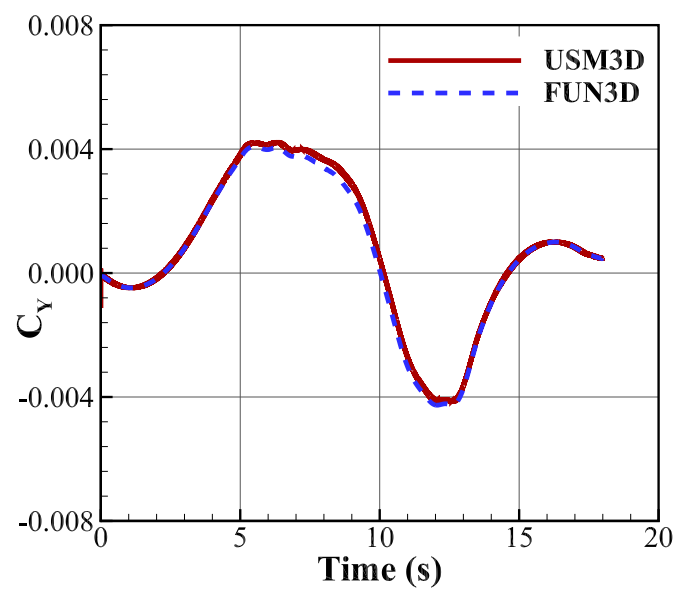

(e) Side Force

Fig. 9 Comparison of USM3D and FUN3D flow solvers for SACCON half Lazy-8 maneuver. Grids 10Mc and 10Mn. $M_{\infty}=0.2789$, Re $e_{\text {cref }}=1.57 \times 10^{6}, \Delta t^{*}=0.10, n_{\text {sub }}=10$. 
The impact of grid density on $C_{m}$ and $C_{n}$ is shown in Fig. 10 for both codes using the 5-, 10-, and 20-element grids. The solutions were generated using a characteristic time step of $\Delta t^{*}=0.10$ and $n_{s u b}=10$ subiterations. The USM3D solutions are generally grid converged with 10- and 20-million elements. The grid sensitivities are even less for the FUN3D solutions. From these results, the 10-million element grids were chosen for further parameter sensitivity assessments.

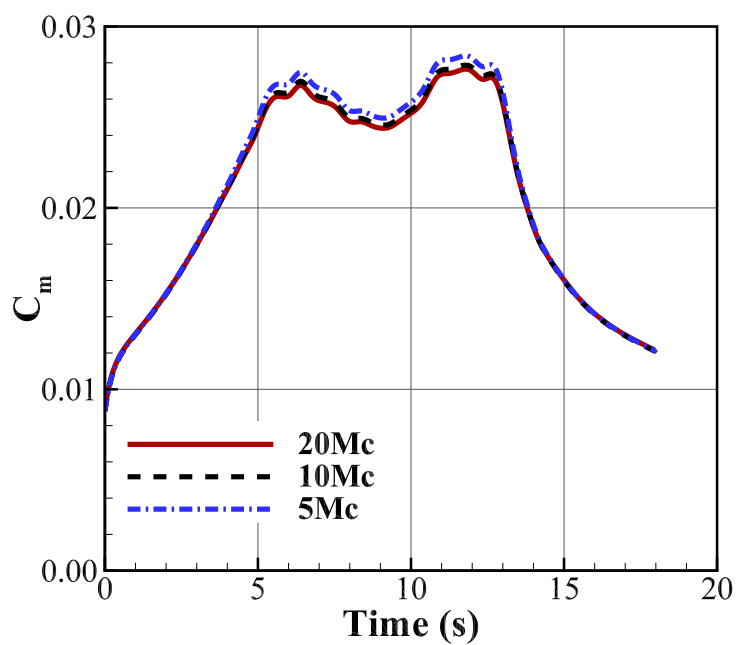

(a) USM3D Pitching Moment

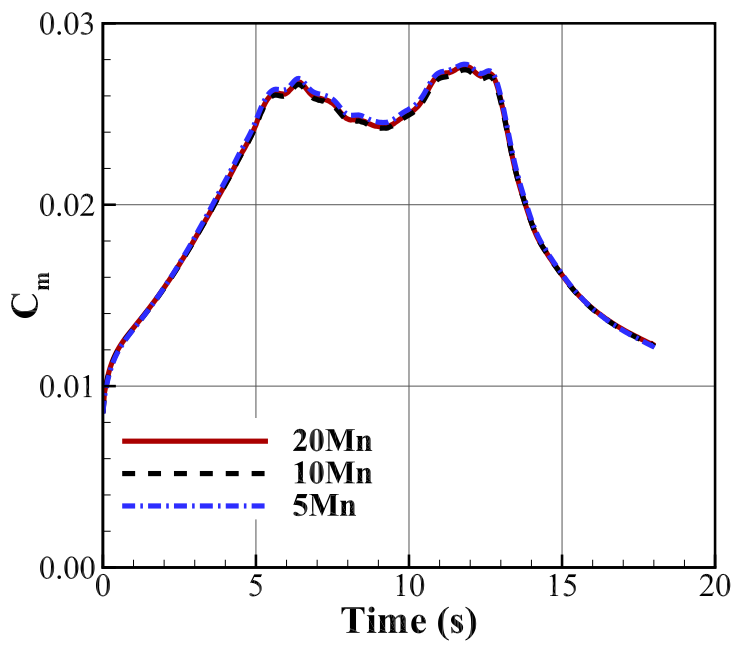

(c) FUN3D Pitching Moment

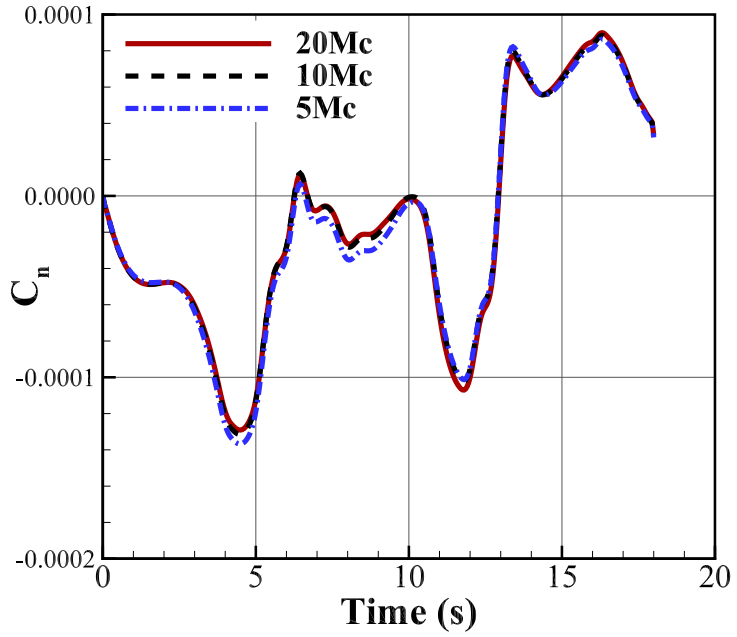

(b) USM3D Yawing Moment

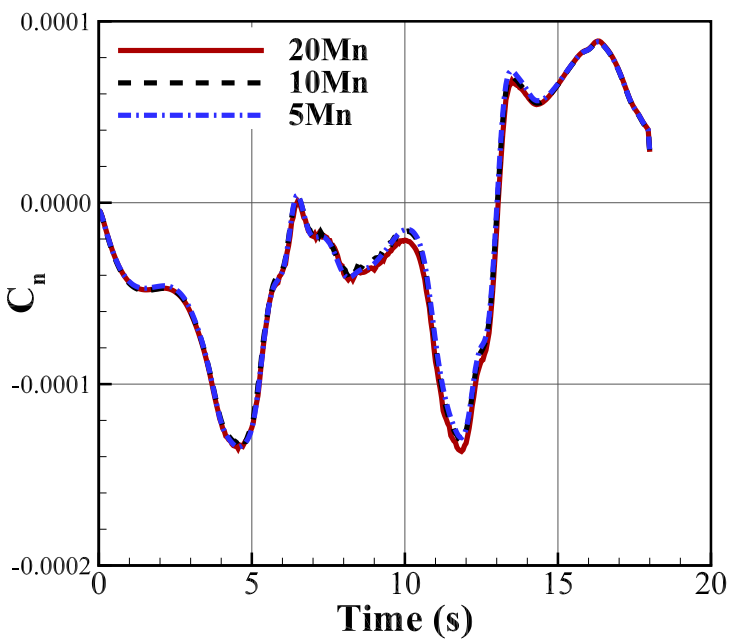

(d) FUN3D Yawing Moment

Fig. 10 Effect of grid density on time-varying pitch and yaw moment coefficients for SACCON half Lazy-8 maneuver: USM3D upper, FUN3D lower. $M_{\infty}=0.2789, R e_{\text {cref }}=1.57 \times 10^{6}, \Delta t^{*}=0.10, n_{\text {sub }}=10$.

Temporal convergence was assessed with the cases listed in Table 2. The effect of subiterative convergence between time steps is evaluated using Cases 2.1,2.2, and 2.3, where a constant time step of $\Delta t^{*}=0.10$ is maintained to traverse the 18-second half Lazy-8 maneuver in 36,000 time steps. As evident from the first three cases in Table 2, the number of subiterations applied to this constant time step has a proportional impact on the total number of solution iterations and hence, cost. The $C_{m}$ and $C_{n}$ results presented in Fig. 11 suggests that $n_{s u b}=10$ is sufficient for subiterative convergence for both flow solvers. While not shown, this conclusion was further substantiated by examining the subiterative convergence history of the force and moment coefficients for the solutions. 
Table 2 Subiteration and time step parameters for temporal convergence of Half Lazy 8. USM3D/SA/10Mc and FUN3D/SA/10Mn.

\begin{tabular}{cccccc}
\hline \hline Case & $\Delta t(\mathrm{~s})$ & $\Delta t^{*}$ & $N_{\text {sub }}$ & Total Time Steps & Total Iterations \\
\hline 2.1 & $5.00 \mathrm{E}-4$ & 0.10 & 15 & 36,000 & 540,000 \\
\hline 2.2 & $5.00 \mathrm{E}-4$ & 0.10 & 10 & 36,000 & 360,000 \\
2.3 & $5.00 \mathrm{E}-4$ & 0.10 & 5 & 36,000 & 180,000 \\
\hline 2.4 & $2.50 \mathrm{E}-4$ & 0.06 & 10 & 54,000 & 540,000 \\
2.5 & $1.25 \mathrm{E}-4$ & 0.03 & 5 & 108,000 & 540,000 \\
2.6 & $5.00 \mathrm{E}-5$ & 0.01 & 5 & 360,000 & $1,800,000$ \\
\hline \hline
\end{tabular}

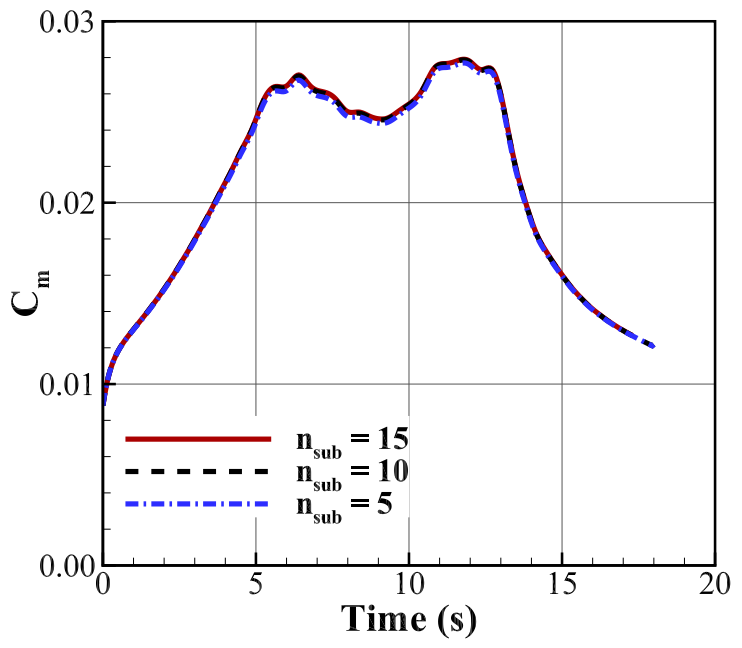

(a) USM3D: Pitching Moment

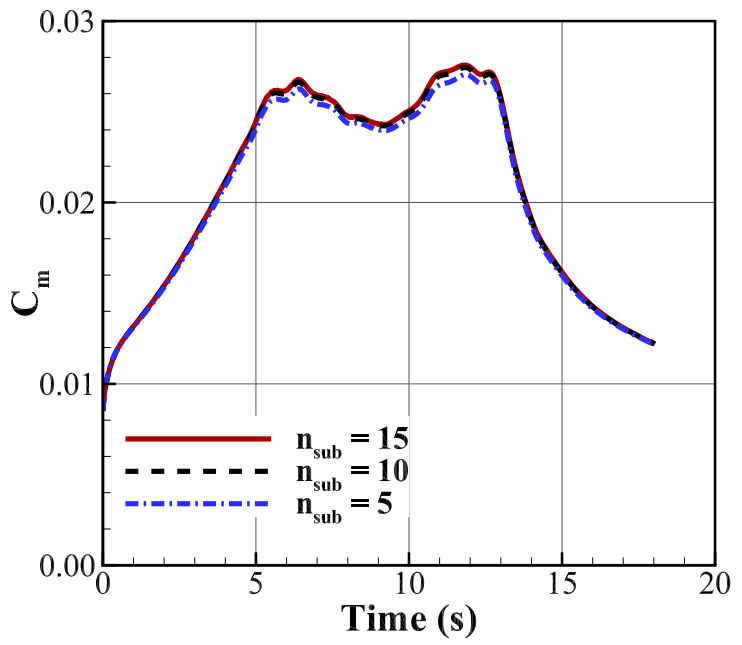

(c) FUN3D: Pitching Moment

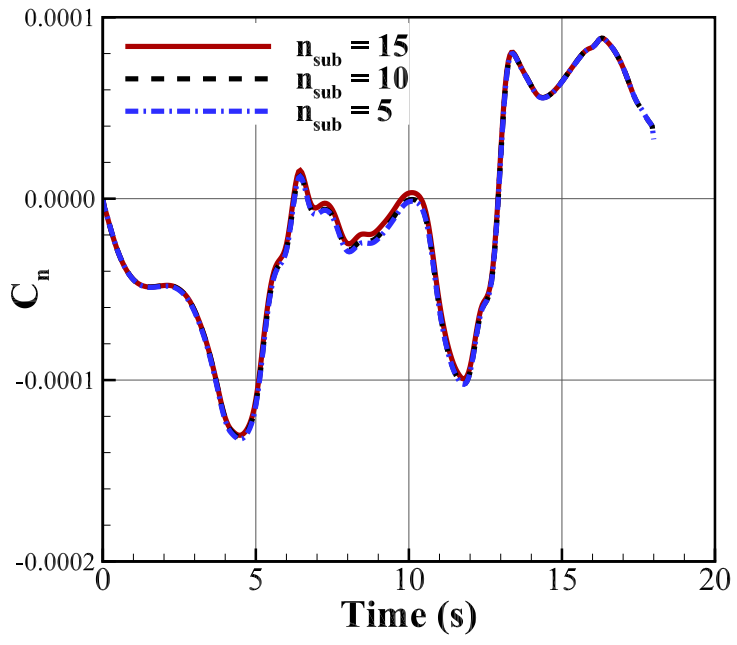

(b) USM3D: Yawing Moment

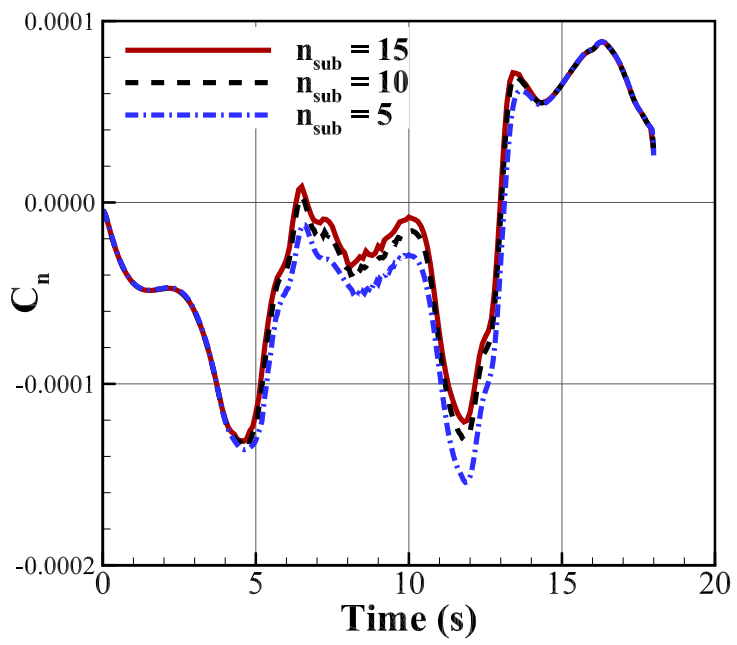

(d) FUN3D: Yawing Moment

Fig. 11 Effect of inner time step subiteration on pitch and yaw moment coefficients for SACCON half Lazy-8 maneuver: USM3D upper, FUN3D lower. Grids 10Mc and 10Mn. $M_{\infty}=0.2789, R_{\text {cref }}=1.57 \times 10^{6}, \Delta t^{*}=0.10$.

The sensitivity to time step size is captured by the Cases $2.1,2,4,2.5$, and 2.6 in Table 2 with $\Delta t^{*}=0.10,0.06,0.03$, 
and 0.01, respectively. A constant number of total solution iterations of 540,000 was maintained for the first three cases as recommended by Ref. [42]. The fourth Case 2.6, with $\Delta t=0.01$ and 1,800,000 total iterations, was included to provide a consistency with the time step to be used in the following indicial response solutions. As is evident in Fig. 12 . the time step size has negligible impact on the solution for the half Lazy-8 maneuver.

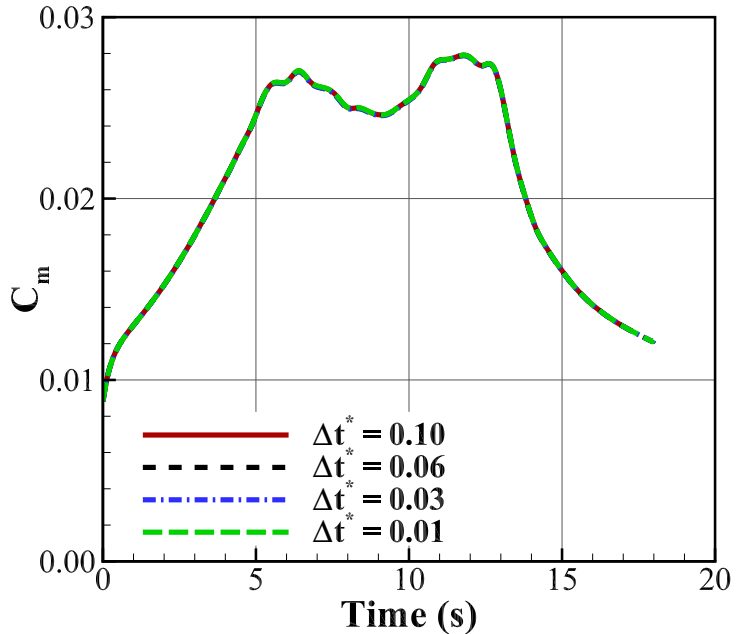

(a) USM3D: Pitching Moment

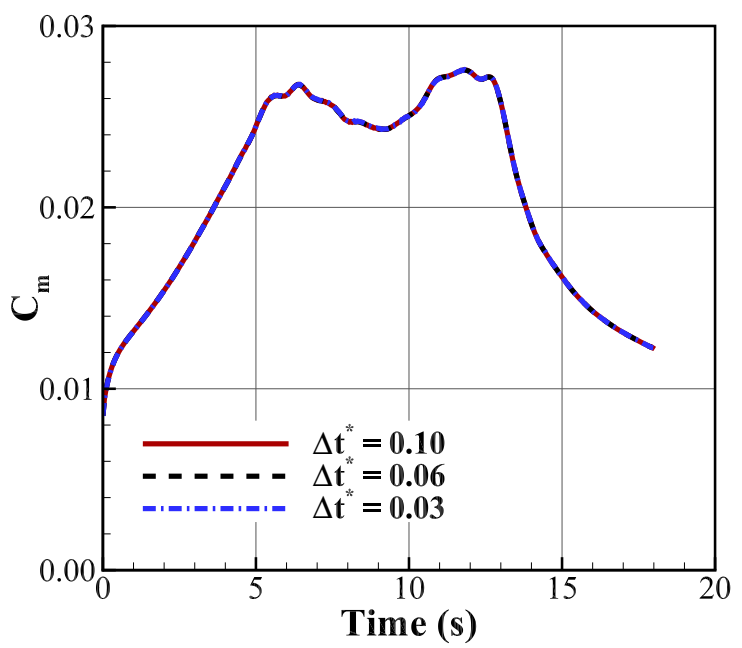

(c) FUN3D: Pitching Moment

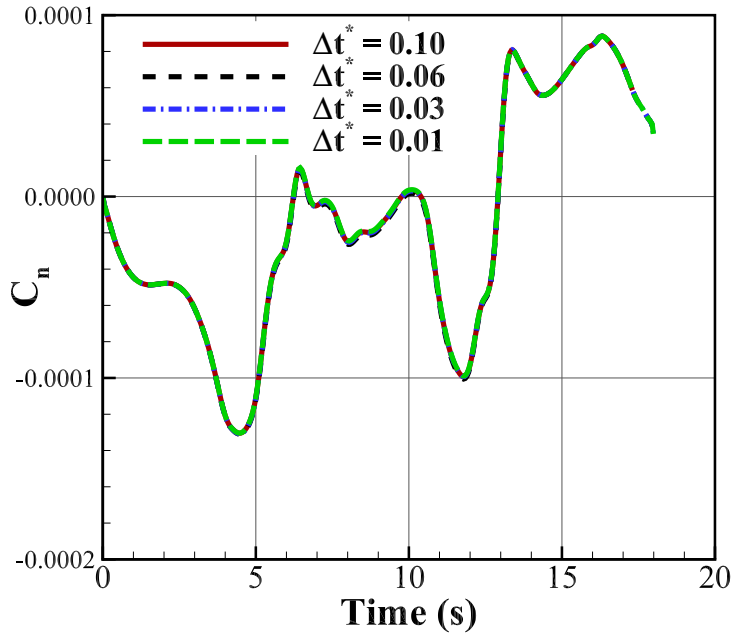

(b) USM3D: Yawing Moment

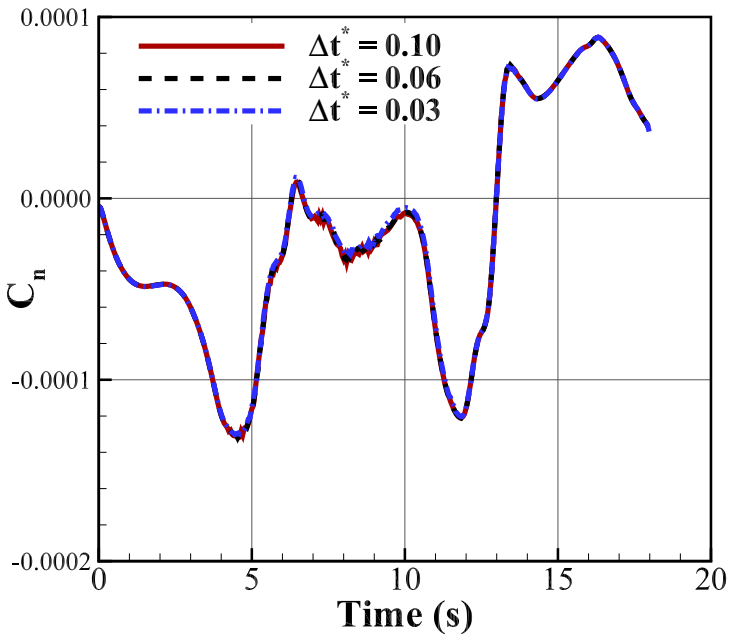

(d) FUN3D: Yawing Moment

Fig. 12 Effect of time step on pitch and yaw moment coefficients for SACCON half Lazy-8 maneuver: USM3D upper, FUN3D lower. Grids $10 \mathrm{Mc}$ and $10 \mathrm{Mn} . M_{\infty}=0.2789$, $R e_{\text {cref }}=1.57 \times 10^{6}$.

\section{Generation of Indicial Response Solutions}

To insure consistency with the indicial response sampling of Ref. [14], a set of 33 flight space points were sampled within the variable ranges $\alpha_{0}=\left[0^{\circ}-10^{\circ}\right]$ and $M=[0.05-0.30]$ as depicted in Table 3. While the matrix of Ref. [14] spanned $M$ from 0.1 to 0.5 , the half Lazy- 8 and Immelmann maneuvers provided by the USAFA for the present study covered the range of $M$ from 0.07 to 0.28 , assuming sea level speed of sound of $340.3 \mathrm{~m} / \mathrm{s}$. Hence, the selected Mach numbers of $0.05,0.15$, and 0.30 .

The indicial response solutions are generated by imposing through solid-body grid motion a sudden step change in $\alpha, \beta$, or angular rates from a converged static solution at each flow condition in Table 3 Static solutions from the 
sensitivity study in SectionVI.B.1 are used for this purpose. The grid-motion trajectory files are created by applying the vehicle-state inputs at each Mach number, in Table 4, to the transformations noted in Appendix A. The incremental steps are defined as $\Delta \alpha=1^{\circ}, \Delta \beta=1^{\circ}$, and $\Delta p=\Delta q=\Delta r=1 \mathrm{rad} / \mathrm{s}=180 / \pi \mathrm{deg} / \mathrm{s}$.

Table 3 Matrix of 75 indicial step cases for input to the kriging surrogate model.

\begin{tabular}{cccc}
\hline \hline$\alpha_{0}, \operatorname{deg}$ & $M=0.05$ & $M=0.15$ & $M=0.30$ \\
\hline 0 & $\Delta \alpha, \Delta \beta, \Delta p, \Delta q, \Delta r$ & $\Delta \alpha, \Delta \beta, \Delta p, \Delta q, \Delta r$ & $\Delta \alpha, \Delta \beta, \Delta p, \Delta q, \Delta r$ \\
1 to 10 & $\Delta \alpha, \Delta \beta$ & $\Delta \alpha, \Delta \beta$ & $\Delta \alpha, \Delta \beta$ \\
\hline \hline
\end{tabular}

Table 4 Input parameters to transformations in Appendix A for creating indicial trajectories $(t>0)$.

\begin{tabular}{ccccccc}
\hline \hline Indicial & $\alpha, \operatorname{deg}$ & $\beta, \operatorname{deg}$ & $\phi, \operatorname{deg}$ & $\theta, \operatorname{deg}$ & $\psi, \operatorname{deg}$ & $M_{0}$ \\
\hline$\alpha$-step & $\alpha_{0}+1$ & 0 & 0 & 0 & 0 & $0.05,0.15,0.30$ \\
$\beta$ - step & $\alpha_{0}$ & 1 & 0 & 0 & 0 & $0.05,0.15,0.30$ \\
$\Delta p$ - step & 0 & 0 & $\Delta p \cdot t$ & 0 & 0 & $0.05,0.15,0.30$ \\
$\Delta q$ - step & 0 & 0 & 0 & $\Delta q \cdot t$ & 0 & $0.05,0.15,0.30$ \\
$\Delta r$-step & 0 & 0 & 0 & 0 & $\Delta r \cdot t$ & $0.05,0.15,0.30$ \\
\hline \hline
\end{tabular}

For an $\Delta \alpha$-step and $\Delta \beta$-step, the initial time step is set to $\alpha=\alpha_{0}, \beta=0^{\circ}$, and $\phi=\theta=\psi=0^{\circ}$. For a $\Delta \alpha$-step, this produces a pure translation motion downward and slightly aft to maintain $\Delta q=0 \mathrm{rad} / \mathrm{s}$. For a $\Delta \beta$-step, this imposes a pure translation to the right and slightly aft to maintain $\Delta r=0 \mathrm{rad} / \mathrm{s}$. The $\Delta p-, \Delta q-$, and $\Delta r-$ steps are initialized by setting $\alpha=\beta=\phi=\theta=\psi=0^{\circ}$ for the initial time step. For a $\Delta p$-step, this results in an impulsive roll of the grid about the body axis. For a $\Delta q$-step, a constant-rate pitch rotation of the grid coupled with an upward and aft translation along a curved path that maintains $\alpha=\beta=0^{\circ}$ throughout the motion. For a $\Delta r$-step, there is a constant-rate yaw rotation of the grid coupled with a sideways and aft translation, such that $\alpha=\beta=0^{\circ}$ is maintained during the motion.

The indicial step responses were computed with USM3D and FUN3D using the temporal parameters presented in Table 5. All responses, shown in Appendix B, were advanced for 500 time steps with $\Delta t^{*}=0.011$ to an $s_{\max }=10$ where asymptotic convergence to steady state was achieved. The abscissa on those plots is compressed to a maximum of $s=4$ to illuminate the initial transients.

\section{Assessment of Reduced-Order Model (ROM)}

The utility of the indicial ROM is explored through a series of parameter sensitivity studies while SACCON performs various maneuvers. Model accuracy is evaluated by comparisons with the full-order CFD flight maneuver solutions. The goal is to gain an understanding of the ROM behavior, which leads to application guidelines that produce an acceptable level of accuracy with the least amount of model generation time and run time. There are several parameters that have a direct effect on the cost and accuracy of the model. For example, the density of the surrogate model, i.e., the number of indicial responses needed for $\alpha, \beta$, and $M$, has a direct impact on both the accuracy and cost. In practice, the CFD time step size for the indicial responses should be as large as practical, and the duration of the response as small as possible. Similarly, the time step size while integrating the trajectory in the ROM manifests in a trade-off between dynamic resolution and model run time.

\section{Roll, pitch, and yaw forced oscillations}

Typical S\&C databases may require forced oscillations at multiple flight conditions, amplitudes, and frequencies. The computational efficiency of the ROM provides an opportunity to supply high-fidelity simulation data at a fraction of the cost as would be required for full-order solutions, but with sufficient modeling accuracy. To evaluate the viability of such an approach, a series of forced sinusoidal oscillations about the roll, pitch, and yaw axes were simulated using the NASA USM3D and FUN3D flow solvers for comparison to ROM predictions. The salient scaling parameter for the FO simulations is reduced frequency, $k=2 \pi f L_{r e f} / U_{\infty}$, where $L_{r e f}=c_{r e f}$ for pitch oscillation and $L_{r e f}=b / 2$ for roll and yaw oscillations. Each of the cases were initialized from a well-converged static simulation and subsequently 
Table 5 Temporal parameters for indicial step responses.

\begin{tabular}{ccccccccc}
\hline \hline Indicial & Mach & $\Delta t(\mathrm{~s})$ & $\Delta t^{*}$ & $s_{\max }$ & $N_{\text {sub }}$ & Total Time Steps & Total Iterations & No. Cases \\
\hline$\Delta \alpha$ & 0.30 & $5.0 \mathrm{E}-05$ & 0.011 & 10 & 10 & 500 & 5000 & 11 \\
$\Delta \alpha$ & 0.15 & $1.0 \mathrm{E}-4$ & 0.011 & 10 & 10 & 500 & 5000 & 11 \\
$\Delta \alpha$ & 0.05 & $3.0 \mathrm{E}-04$ & 0.011 & 10 & 10 & 500 & 5000 & 11 \\
\hline$\Delta \beta$ & 0.30 & $5.0 \mathrm{E}-05$ & 0.011 & 10 & 10 & 500 & 5000 & 11 \\
$\Delta \beta$ & 0.15 & $1.0 \mathrm{E}-4$ & 0.011 & 10 & 10 & 500 & 5000 & 11 \\
$\Delta \beta$ & 0.05 & $3.0 \mathrm{E}-04$ & 0.011 & 10 & 10 & 500 & 5000 & 11 \\
\hline$\Delta p$ & 0.30 & $5.0 \mathrm{E}-05$ & 0.011 & 10 & 10 & 500 & 5000 & 1 \\
$\Delta p$ & 0.15 & $1.0 \mathrm{E}-4$ & 0.011 & 10 & 10 & 500 & 5000 & 1 \\
$\Delta p$ & 0.05 & $3.0 \mathrm{E}-04$ & 0.011 & 10 & 10 & 500 & 5000 & 1 \\
\hline$\Delta q$ & 0.30 & $5.0 \mathrm{E}-05$ & 0.011 & 10 & 10 & 500 & 5000 & 1 \\
$\Delta q$ & 0.15 & $1.0 \mathrm{E}-4$ & 0.011 & 10 & 10 & 500 & 5000 & 1 \\
$\Delta q$ & 0.05 & $3.0 \mathrm{E}-04$ & 0.011 & 10 & 10 & 500 & 5000 & 1 \\
\hline$\Delta r$ & 0.30 & $5.0 \mathrm{E}-05$ & 0.011 & 10 & 10 & 500 & 5000 & 1 \\
$\Delta r$ & 0.15 & $1.0 \mathrm{E}-4$ & 0.011 & 10 & 10 & 500 & 5000 & 1 \\
$\Delta r$ & 0.05 & $3.0 \mathrm{E}-04$ & 0.011 & 10 & 10 & 500 & 5000 & 1 \\
\hline \hline
\end{tabular}

advanced in time with a characteristic time step of $\Delta t^{*}=0.011$. Two cycles of motion were simulated for each case to allow for the initial transient response to decay, leading to a converged hysteresis loop for the second cycle of motion.

Three forced pitch oscillation cases were simulated using the USM3D and FUN3D flow solvers. Each oscillation was initialized at a $5^{\circ}$ mean angle of attack and $0^{\circ}$ sideslip angle, and simulated with a $5^{\circ}$ pitching amplitude. The first and second cases were simulated with a common Mach number, $M=0.15$, but at different reduced frequencies, $k=[0.06,0.12]$. These cases allow for the ROM prediction capability to be assessed for longitudinal motions of varying flow unsteadiness. Because the cases were simulated at a Mach number for which indicial responses were sampled, a nonlinear ROM accounting only for variations in angle of attack is necessary to predict the response across a wide range of angles of attack, $\alpha=\left[0^{\circ}-10^{\circ}\right]$. The second and third cases were simulated using a common reduced frequency, $k=0.12$, but at different Mach numbers, $M=[0.15,0.20]$. Since the third case is simulated at a Mach number not previously sampled in generating the ROM, it requires the complete nonlinear ROM given by Eq. 3 , which accounts for variations with respect to both angle of attack and Mach number.

Figure 13 illustrates a comparison between CFD and ROM results for each of the forced pitch oscillation cases (top to bottom) using the USM3D (left) and FUN3D (right) flow solvers. While the ROM was used to generate predictions for both the lift and pitching moment coefficients, only the pitching moment results are shown for brevity with similar prediction trends found for the lift coefficient. Overall, the ROM predictions were shown to be in close agreement with the full-order CFD simulations for each of the forced pitch oscillations, in which a pitching moment curve exhibiting a counter-clockwise hysteresis loop is observed. In every case, the ROM is able to accurately resolve the aerodynamic response, including the effect of the initial grid motion, where both the magnitude of the initial peak and the transient decay are exactly predicted by the ROM.

Between the first and second cases, the damping effect of increased reduced frequency (pitch rate) is captured correctly in the thickening of the hysteresis loops. The minimum and maximum pitching moment values are not affected by reduced frequency.

Between the second and third cases, an increase in Mach number yields similar pitching moment results with the primary difference being a damping of the initial transients at the higher Mach number due to an increase in the propagation speed of pressure disturbances. Despite not directly sampling the indicial responses at $M=0.2$, the ROM is able to predict the damping of the initial transient and a pitching moment loop comparable to CFD simulation data.

For each of the pitch oscillation cases, deviations between the ROM and CFD results were observed during the down-swing motion at lower angles of attack. This trend was observed for both flow solvers at several frequencies and Mach numbers. One of the widely used assumptions of the reduced-order model is that the step response is symmetric for positive and negative changes in angle of attack, i.e., the response would be identical in initial peak and transient 
behavior, but opposite in sign. In observation of the results, it would appear that this assumption may be invalid for the SACCON UCAV used in the present study. This is not a particularly surprising result as modern flight vehicle configurations are not generally symmetric about the lateral planes of motion. This observation leads to the suggestion that future ROM efforts should explore the sampling requirements for positive and negative step responses within positive and negative angles of attack regimes for accurate $S \& C$ predictions.

Next, three forced yaw oscillation cases were simulated using the USM3D and FUN3D flow solvers. The SACCON UCAV is a thin tailless aircraft, where the lateral forces and moments are very small in magnitude. Hence, the following examples represent a vigorous test of the ROM methodology. Each oscillation was initialized at a $7.5^{\circ}$ angle of attack and $0^{\circ}$ mean sideslip angle, and simulated with a $5^{\circ}$ yawing amplitude. The first and second cases were simulated with a common Mach number, $M=0.15$, but at different reduced frequencies, $k=[0.10,0.19]$. These cases allow for the ROM prediction capability to be assessed for lateral motions of varying flow unsteadiness. Because the yaw oscillation cases maintain a zero angle of attack and are simulated at a previous sampled Mach number, a linear variant of the ROM given by Eq. 4 is used for model predictions, where no dependencies on angle of attack or Mach number are necessary for modeling. The second and third cases were simulated using a common reduced frequency, $k=0.19$, but at different Mach numbers, $M=[0.15,0.20]$. Since the third case is simulated at a Mach number not previously sampled in generating the ROM, it requires a simplified variant of the nonlinear ROM given by Eq. 3 , where only a dependency on Mach number is required for modeling.

Figure 14 illustrates a comparison between CFD and ROM results for each of the forced yaw oscillation cases (top to bottom) using the USM3D (left) and FUN3D (right) flow solvers. While the ROM was used to generate predictions for the side force, rolling moment, and yawing moment coefficients, only the yawing moment results are shown with similar prediction trends found for the other coefficients. Overall, the ROM predictions were shown to be in general agreement with the full-order CFD simulations for each of the forced yaw oscillations, in which a yawing moment curve exhibiting a clockwise hysteresis loop is observed. In every case, the ROM is able to accurately resolve the aerodynamic response due to the initial grid motion, where both the magnitude of the initial peak and the transient decay are well predicted by the ROM.

Between the first and second cases, the damping effect of increased reduced frequency (yaw rate) is captured correctly in the thickening of the hysteresis loops. The minimum and maximum yawing moment values are not affected by reduced frequency. However, a discrepancy is observed in ROM predictions of the minimum and maximum yawing moment coefficient values. Here, the coefficient maximum (at minimum sideslip angle) is overpredicted, and the coefficient minimum (at maximum sideslip angle) is underpredicted, as evidenced by the clockwise tilt of the hysteresis loops in Fig 14. For the linear ROM predictions, the calculated coefficient extreme values are largely a product of the minimum/maximum sideslip angle values (e.g., $\beta=5^{\circ}$ ) and the final steady-state coefficient value, $C_{l_{\beta}} \mid t=\infty$, from the converged indicial response in stepping from $\beta=0^{\circ}$ to $\beta=1^{\circ}$. The observed differences between the linear ROM predictions and the CFD simulation data may be attributed to an unaccounted nonlinear dependency on sideslip angle for SACCON yawing oscillations. The consequence of such a nonlinearity manifests in a need to sample indicial responses with respect to sideslip angle, the first term in Eq. 4 as a function of not only angle of attack and Mach number, but also sideslip angle.

Between the second and third cases, an increase in Mach number yields similar yawing moment results with the primary difference being a damping of the initial transients at the higher Mach number, consistent with the response behavior observed in the pitch oscillation simulations. Again, the ROM is able to predict the damping of the initial transient and a yawing moment loop comparable to CFD simulation data. The differences in minimum and maximum yawing moment coefficient values between the ROM and CFD results are seen again at the higher Mach number. Such a result is expected given that the differences are likely attributed to insufficient modeling of state parameter dependencies. Additionally, it is important to note that in the ROM formulation given by Eq. 4 , the indicial responses with respect to the roll and yaw rates are assumed to be independent of angle of attack. Consistent with this assumption, indicial responses sampled at $\alpha=0^{\circ}$ were used in predicting the contributions from roll and yaw rates. However, the yaw oscillation cases in the present study were simulated at $\alpha=7.5^{\circ}$, and thus, may illustrate a non-negligible dependency on angle of attack for the angular rates, as originally assumed for sideslip indicial responses. 


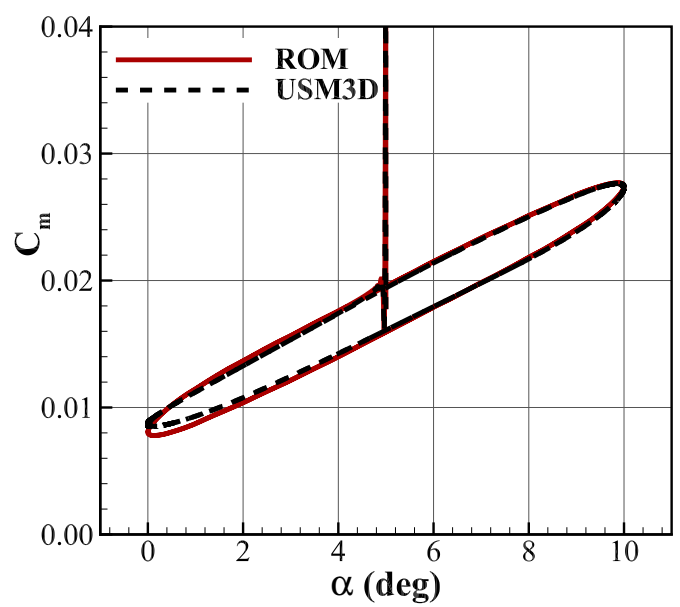

(a) USM3D - FO Pitch ( $\mathrm{k}=0.06, \mathrm{M}=0.15)$

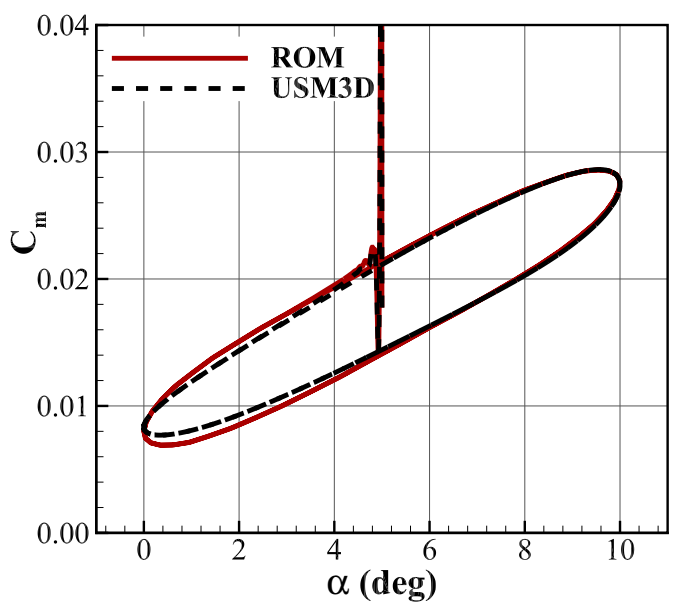

(c) USM3D - FO Pitch (k=0.12, M=0.15)

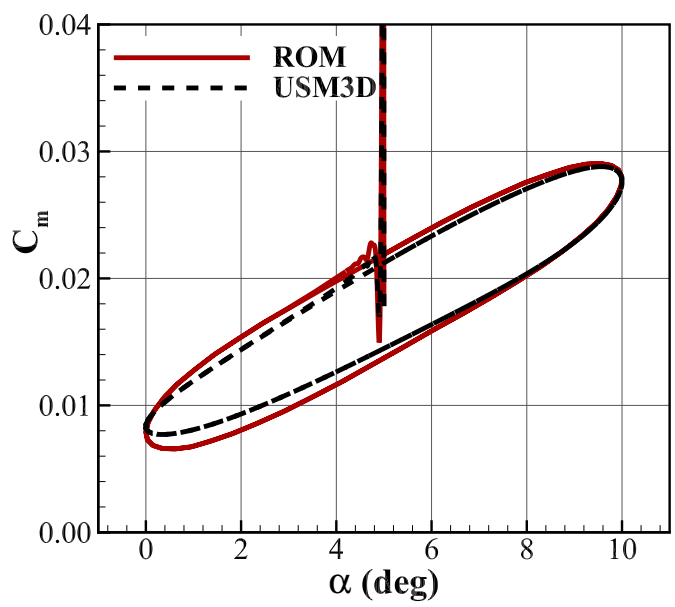

(e) USM3D - FO Pitch (k=0.12, M=0.20)

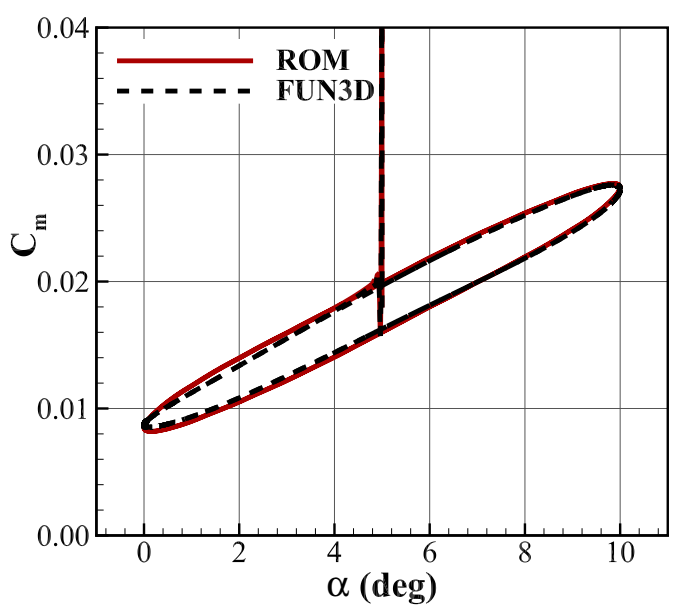

(b) FUN3D - FO Pitch (k=0.06, M=0.15)

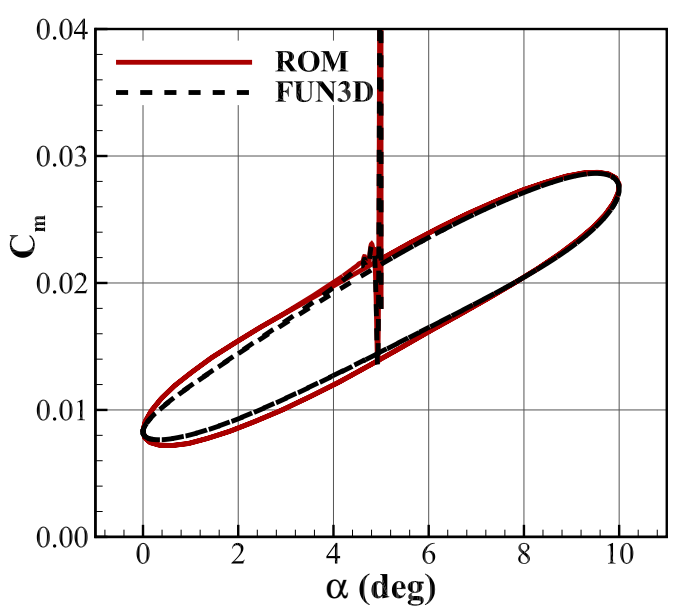

(d) FUN3D - FO Pitch (k=0.12, M=0.15)

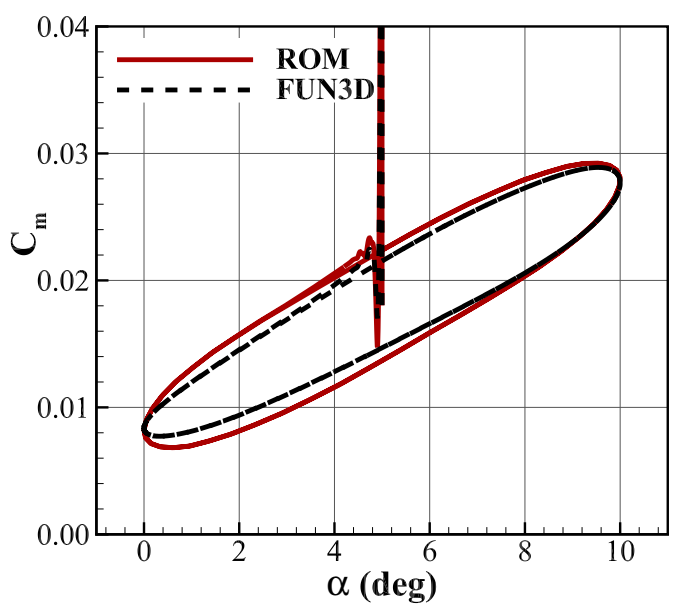

(f) FUN3D - FO Pitch (k=0.12, M=0.20)

Fig. 13 Comparison of ROM and CFD pitching moment results for SACCON forced pitching oscillations: USM3D left, FUN3D right. 10Mc and 10Mn grid. $\Delta t^{*}=0.01, \operatorname{Re}_{\text {cref }}=1.57 \times 10^{6}$. 


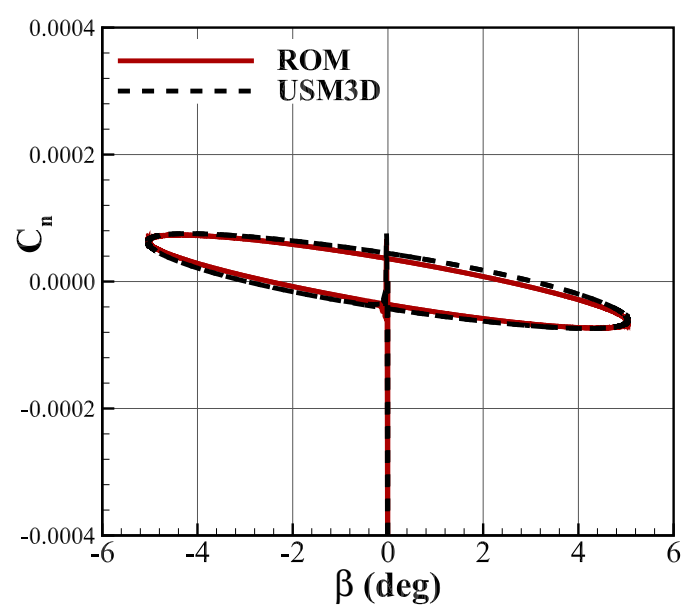

(a) USM3D - FO Yaw ( $\mathrm{k}=0.10, \mathrm{M}=0.15)$

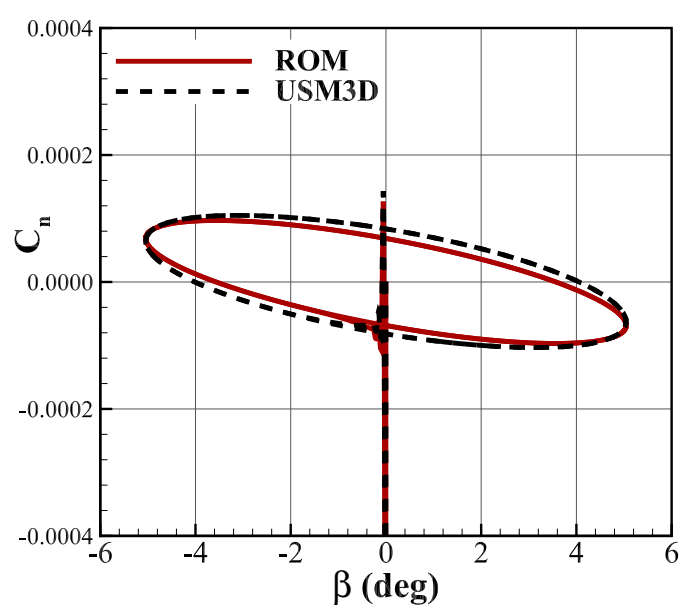

(c) USM3D - FO Yaw (k=0.19, M=0.15)

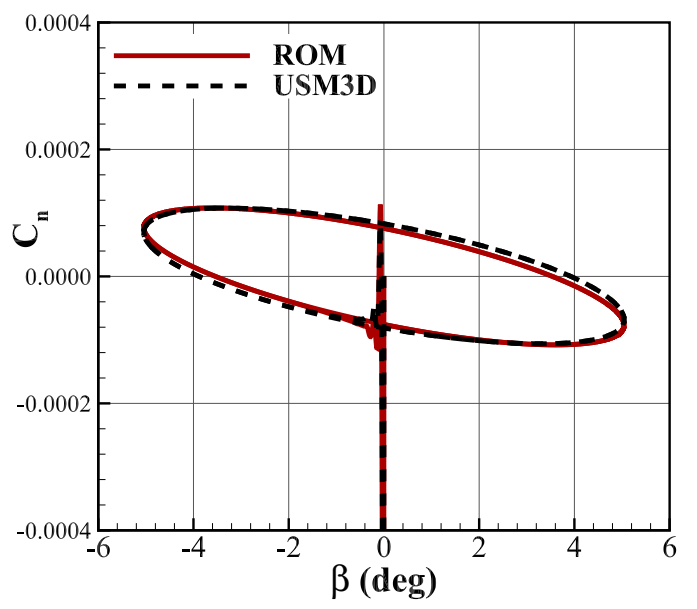

(e) USM3D - FO Yaw $(\mathrm{k}=0.19, \mathrm{M}=0.20)$

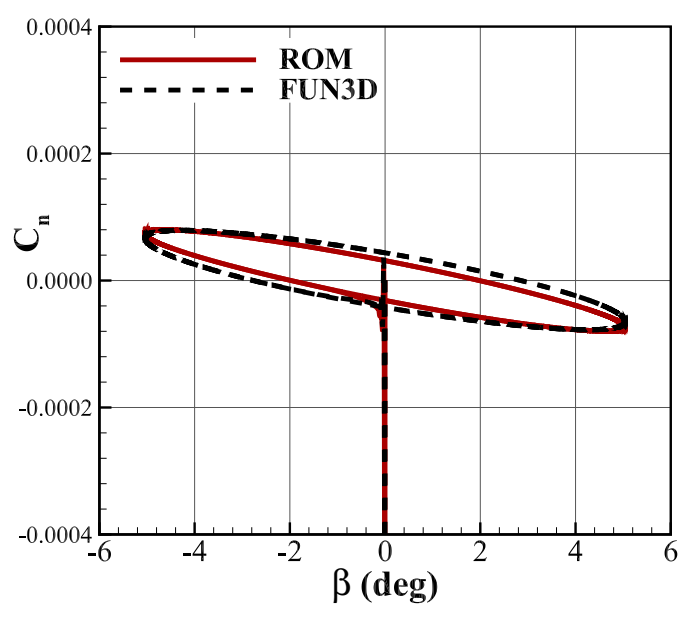

(b) FUN3D - FO Yaw (k=0.10, M=0.15)

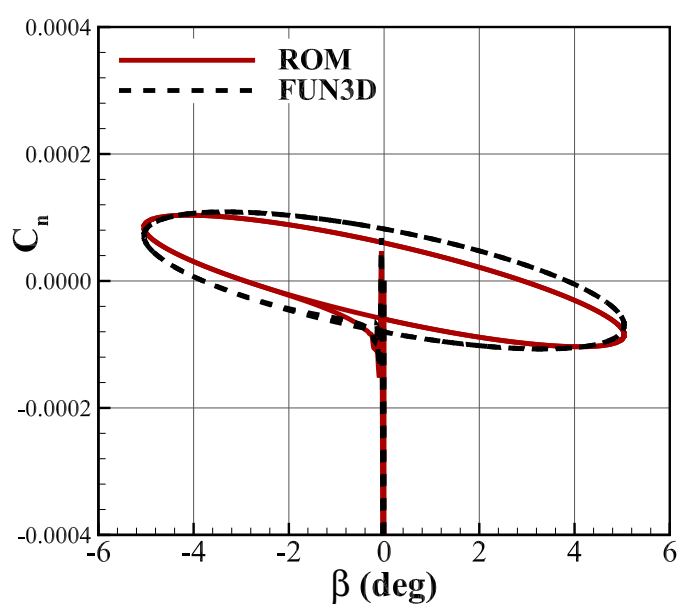

(d) FUN3D - FO Yaw (k=0.19, M=0.15)

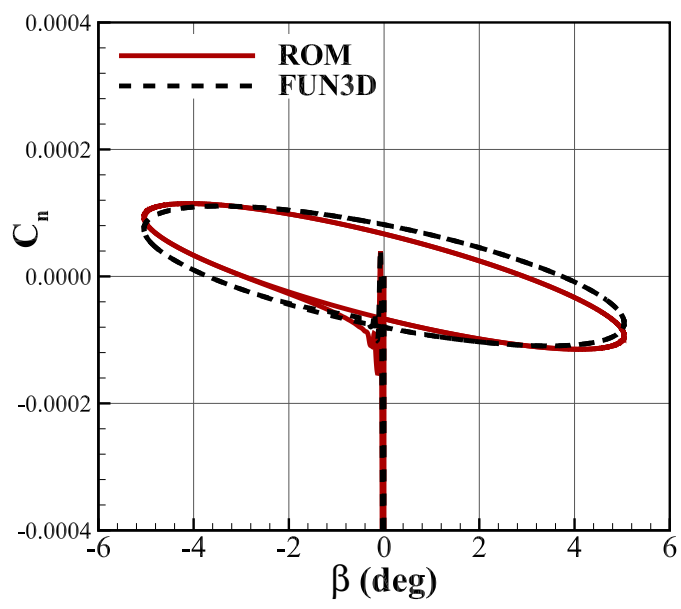

(f) FUN3D - FO Yaw (k=0.19, M=0.20)

Fig. 14 Comparison of ROM and CFD yawing moment results for SACCON forced yawing oscillations: USM3D left, FUN3D right. $10 \mathrm{Mc}$ and $10 \mathrm{Mn}$ grid. $\Delta t^{*}=0.01, \boldsymbol{R e}_{\text {cref }}=1.57 \times 10^{6}$. 
As a final test, two forced rolling oscillation cases were simulated using both flow solvers. Each oscillation was initialized at a $10^{\circ}$ angle of attack and $0^{\circ}$ sideslip angle, and simulated with a $5^{\circ}$ rolling amplitude. The cases were simulated with a common Mach number, $M=0.14$, but at different reduced frequencies, $k=[0.06,0.15]$. Because the roll oscillation cases maintain a constant angle of attack, a linear variant of the ROM given by Eq. 4 is used for model predictions, where only a dependency on Mach number is necessary.

Figure 15 illustrates a comparison between CFD and ROM results for each of the forced roll oscillation cases (top to bottom) using the USM3D (left) and FUN3D (right) flow solvers. While the ROM was used to generate predictions for both the side force, rolling moment, and yawing moment coefficients, only the rolling moment results are shown with similar prediction trends found for the other coefficients. Overall, the ROM predictions were shown to be in general agreement with the full-order CFD simulations for each of the forced roll oscillations, in which a rolling moment curve exhibiting a clockwise hysteresis loop is observed. Similar to the yaw oscillation cases, the ROM was able to accurately resolve the aerodynamic response for each case due to the initial grid motion, where both the magnitude of the initial peak and the transient decay are well predicted by the ROM. An increase in reduced frequency (a faster roll oscillation) results in an expected widening of the loop. The minimum and maximum rolling moment values are not affected by reduced frequency. These ROM results show a general capability to accurately resolve rolling motions encountering varying degrees of flow unsteadiness. In contrast to the yaw oscillations, the ROM was able to accurately predict the minimum and maximum rolling moment coefficient values. Based on these results, it may be concluded that the linear dependency on angle of attack and sideslip angle is a valid assumption for SACCON rolling motions.

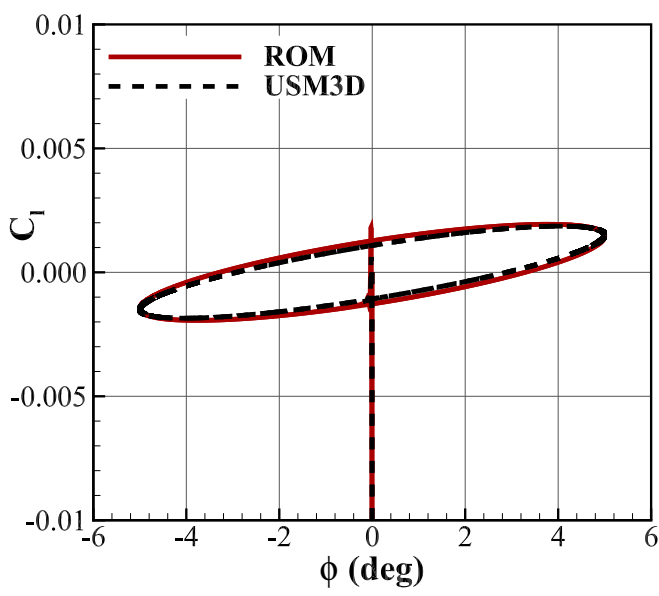

(a) USM3D - FO Roll ( $\mathrm{k}=0.06, \mathrm{M}=0.14)$

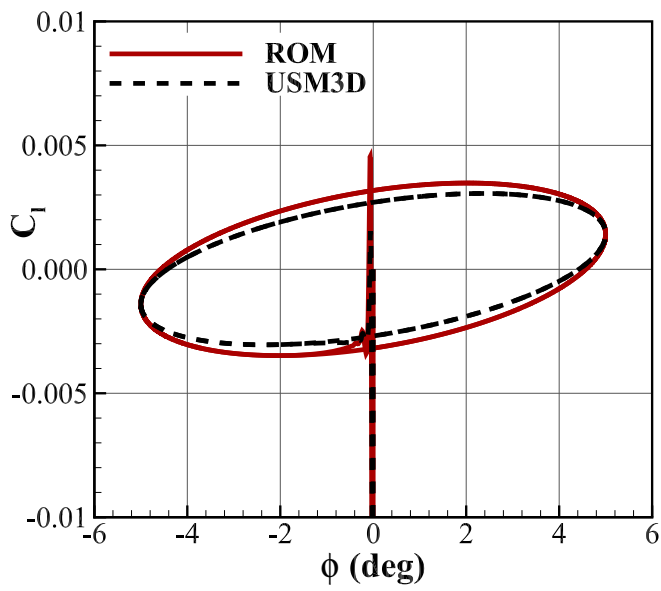

(c) USM3D - FO Roll (k=0.15, M=0.14)

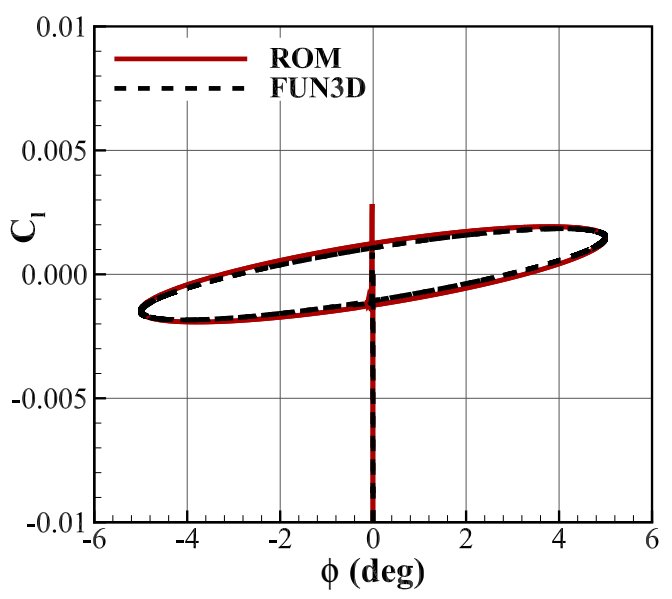

(b) FUN3D - FO Roll (k=0.06, M=0.14)

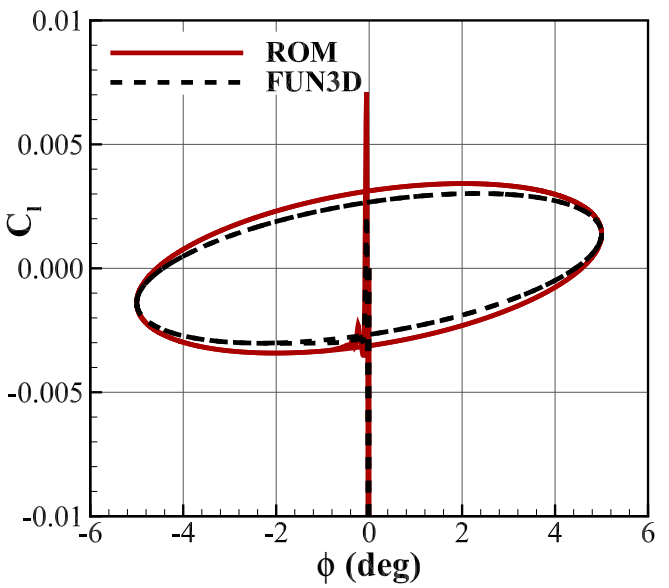

(d) FUN3D - FO Roll (k=0.15, M=0.14)

Fig. 15 Comparison of ROM and CFD rolling moment results for SACCON forced rolling oscillations: USM3D left, FUN3D right. $10 \mathrm{Mc}$ and $10 \mathrm{Mn}$ grid. $\Delta t^{*}=0.01, R_{\text {cref }}=1.57 \times 10^{6}$. 


\section{Half lazy-8 and Immelmann turn maneuvers}

In addition to generating forced oscillation predictions, the nonlinear indicial response ROM is capable of predicting any generalized vehicle trajectory defined in terms of velocity $(\alpha, \beta, M)$ and the Euler orientation angles. If successful, such a capability would provide aircraft designers an ability to "fly" a suite of realistic vehicle trajectories across a range of flight Reynolds and Mach numbers at a fidelity comparable to full-order CFD, but at a fraction of the cost. To demonstrate the prediction quality of the methodology, the half lazy-8 and Immelmann turn maneuvers were modeled using linear and nonlinear variants of the ROM methodology and compared to USM3D and FUN3D simulation results. Each maneuver was simulated using the 10M-element grids and was initialized from a well-converged static simulation. Rigid body motion files were created for each maneuver using the steps outlined in Appendix A, and the maneuvers were subsequently advanced in time with a characteristic time step of $\Delta t^{*}=0.11$ and $n_{\text {sub }}=10$. These same motion files were read in as inputs to the ROM for aerodynamic predictions.

Figures 16 and 17 provide a comparison between the linear ROM, nonlinear ROM, and CFD results for the half Lazy-8 maneuver using the USM3D and FUN3D flow solvers, respectively. The time-varying longitudinal aerodynamic coefficients (lift and pitching moment), and lateral aerodynamic coefficients (rolling moment, yawing moment, and side force) in the body frame of reference are shown over the course of the 18 second maneuver. Here, the ROM results between USM3D and FUN3D compare favorably. Such a result is to be expected given the good agreement of the indicial response solutions in Appendix B. Overall, the half Lazy-8 ROM predictions show qualitative agreement with the CFD simulation data for each coefficient, with the exception of the yawing moment coefficient.

Subfigures 16 and 17 (a-b) show that lift and pitching moment predictions match the CFD solutions fairly well, correctly predicting the coefficient peaks that coincide with the peak angle-of-attack maneuver values. Furthermore, the nonlinear ROM better predicts the lift and pitching moment in comparison to the linear ROM, as it is able to interpolate among the sampled indicial responses to account for the change in angle of attack and Mach number throughout the trajectory. Subfigures 16 and 17 (c-e) show that the rolling moment and side force coefficients are predicted with good accuracy, whereas difficulties are encountered for yawing moment predictions. Given that the SACCON UCAV is a thin tailless aircraft, where the lateral forces and moments are very small in magnitude, it is not surprising that prediction results diminish for the more sensitive aerodynamic quantities. As opposed to the longitudinal coefficient results, significant differences are apparent between the linear and nonlinear ROM results for the lateral coefficients. These differences are most pronounced in the ranges of [6-8] and [11-13] seconds, where the nonlinear ROM can account for the variability in the aerodynamic response at nonzero angles of attack. The discrepancy between the nonlinear ROM predictions and CFD simulation data for the yawing moment coefficient may indicate a requirement for increased sampling and/or the inclusion of a dependency on angle of attack for the angular rate contributions.

Figures 18 and 19 provide a comparison between the linear ROM, nonlinear ROM, and CFD results for the Immelmann turn maneuver using the USM3D and FUN3D flow solvers, respectively. The time-varying longitudinal aerodynamic coefficients (lift and pitching moment), and lateral aerodynamic coefficients (rolling moment, yawing moment, and side force) in the body frame of reference are shown over the course of the 25 second maneuver. Similar to the half Lazy-8 results, the ROM predictions between USM3D and FUN3D compare favorably. Overall, the Immelmann ROM predictions show qualitative agreement with the CFD simulation data for each coefficient, with a consistent exception of the yawing moment coefficient.

Subfigures 18 and 19 (a-b) show that lift and pitching moment predictions match the CFD solutions fairly well, correctly predicting the coefficient variability that correlate with the maneuver's angle-of-attack time history, depicted in Fig 7. As noted in the half Lazy-8 predictions, the nonlinear ROM better predicts the lift and pitching moment in comparison to the linear ROM, as it is able to account for nonlinear dependencies on angle of attack and Mach number. The greatest prediction discrepancy occurs in the range of [7-11] seconds, which coincides with a rapid decrease in Mach number from $M=0.15$ to $M=0.07$. As shown in Appendix B, there is an increasingly transient response in the indicial solutions for lower Mach numbers, suggesting that accurate pitching moment predictions may require increased sampling for Mach numbers approaching incompressible flow levels. Subfigures 18 and 19 (c-e) show that the rolling moment and side force coefficients are predicted with good accuracy, whereas difficulties are again encountered for yawing moment predictions. Differences between the linear and nonlinear ROM results are most pronounced for the rolling moment coefficient, where only the nonlinear ROM is capable of matching the coefficient time histories. The linear and nonlinear ROM both produce reasonable results for the side force coefficient with a general agreement in both magnitude and qualitative behavior. The yawing moment coefficient for both ROM variants is not able to capture the peak CFD levels near $t=10$ seconds. This point along the maneuver coincides with a rapid increase in the yaw and roll angles, shown in Fig.7 as the aircraft simultaneously rolls while performing a steep climb. This observation further supports the idea of exploring the inclusion of a dependency on angle of attack for the lateral angular rate contributions. 


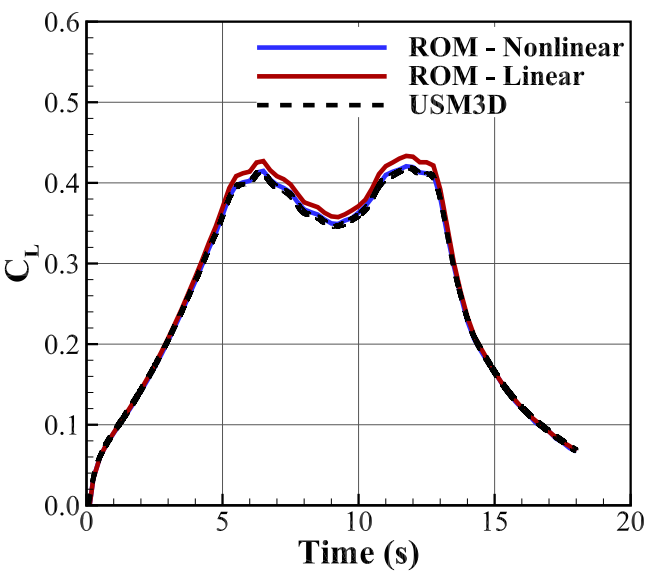

(a) Lift

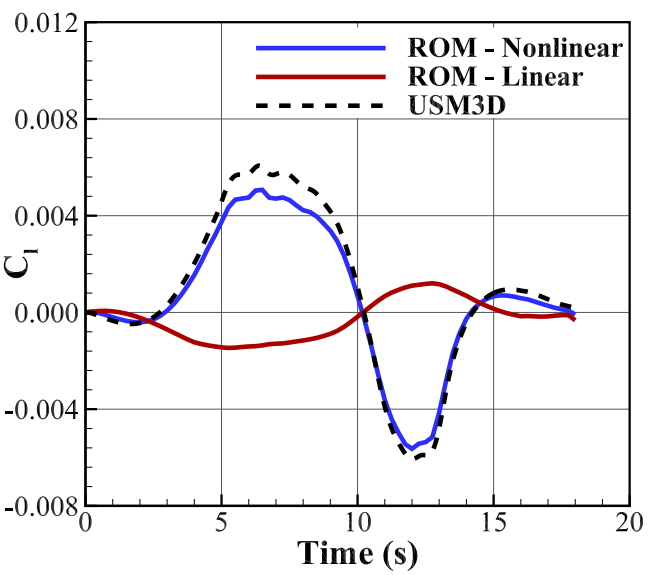

(c) Rolling Moment

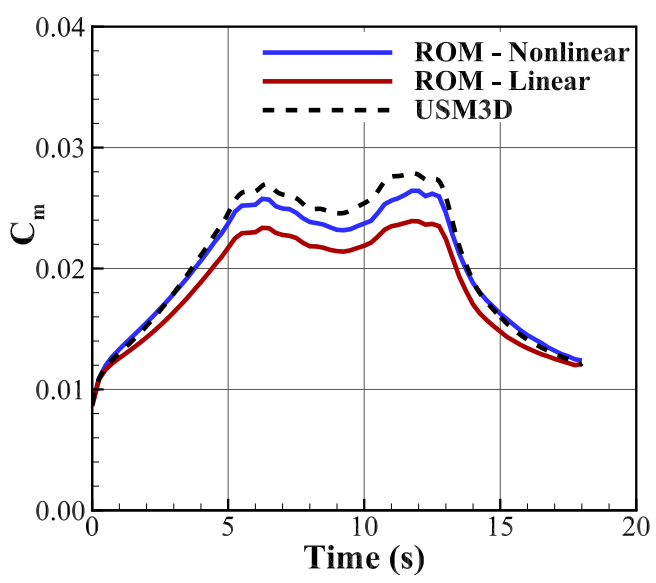

(b) Pitching Moment

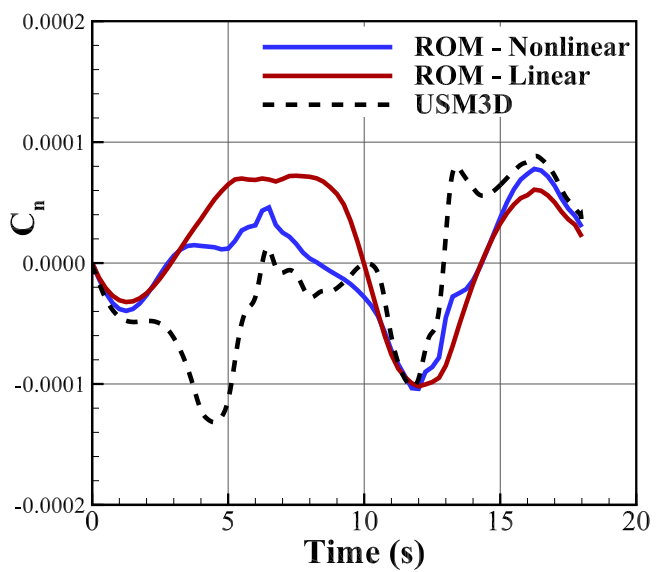

(d) Yawing Moment

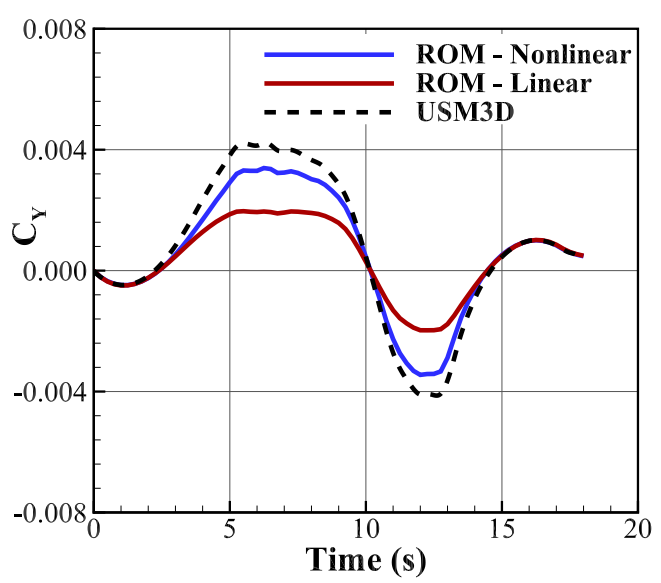

(e) Side Force

Fig. 16 Comparison of ROM and USM3D results for SACCON half Lazy-8 maneuver. 10Mc grid. $M_{\infty}=0.2789, R_{c r e f}=1.57 \times 10^{6}$. 


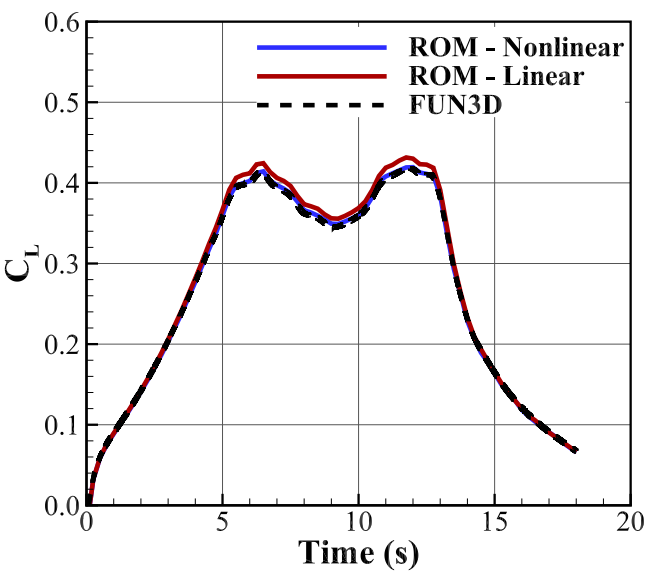

(a) Lift

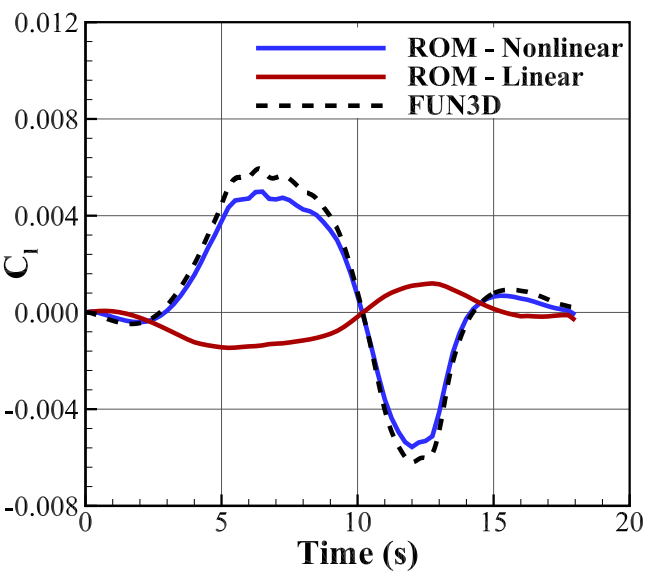

(c) Rolling Moment

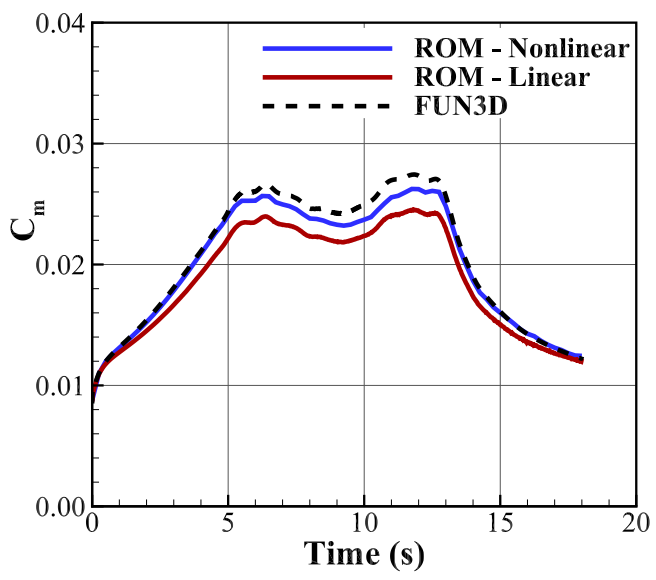

(b) Pitching Moment

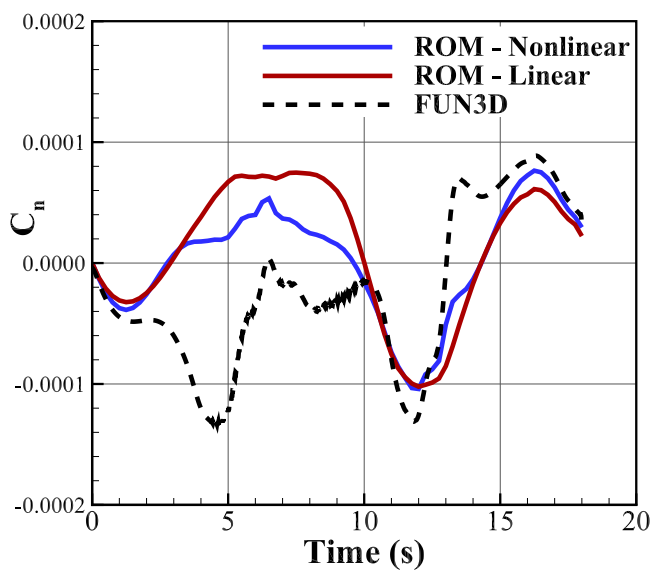

(d) Yawing Moment

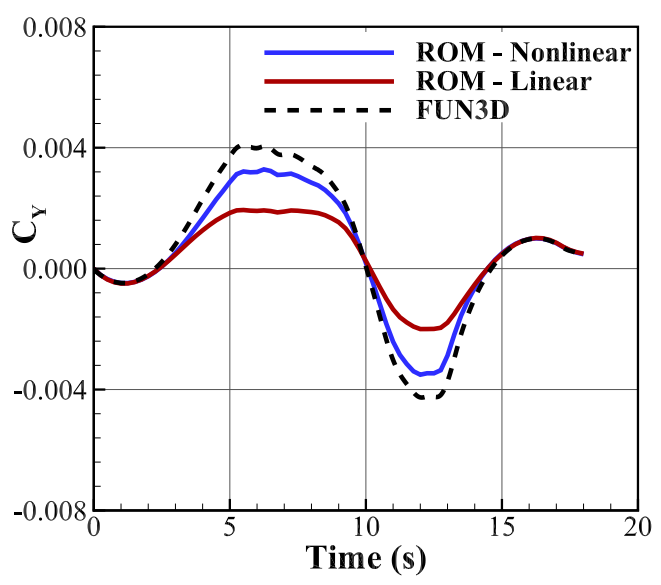

(e) Side Force

Fig. 17 Comparison of ROM and FUN3D results for SACCON half Lazy-8 maneuver. 10Mn grid. $M_{\infty}=0.2789, R_{c r e f}=1.57 \times 10^{6}$. 


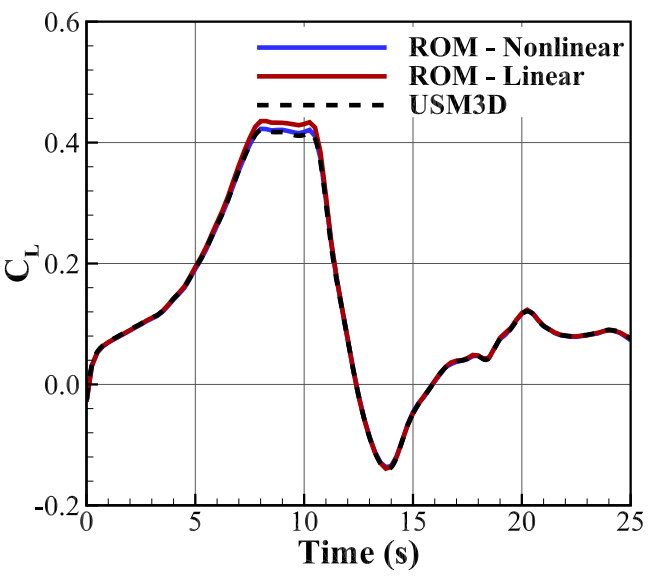

(a) Lift

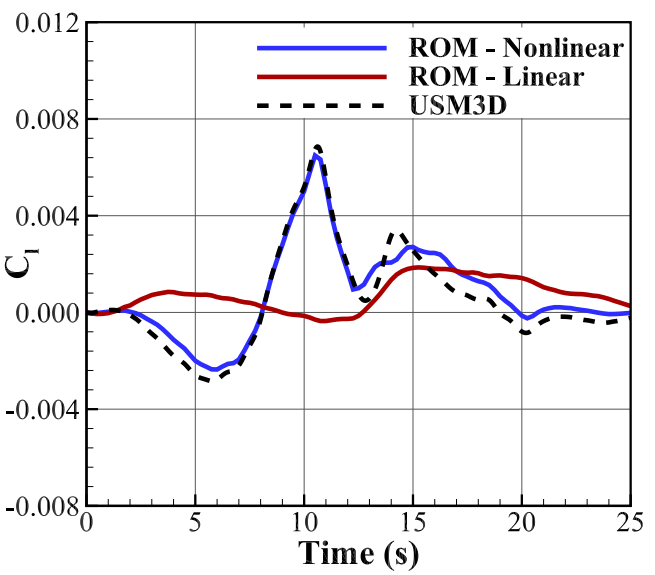

(c) Rolling Moment

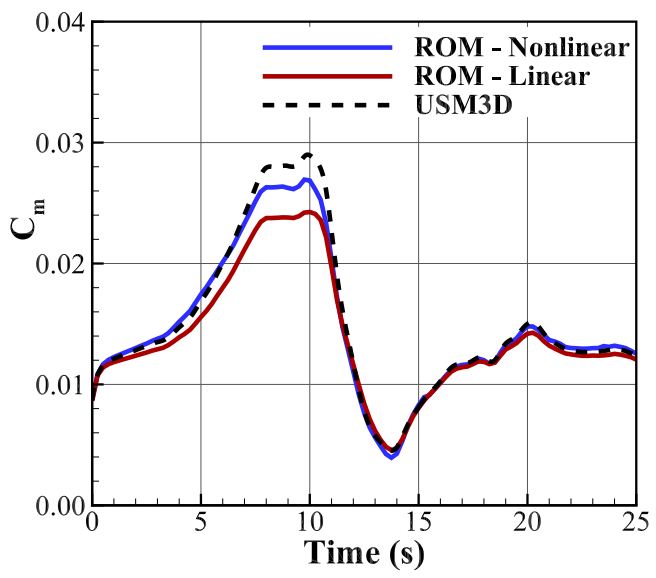

(b) Pitching Moment

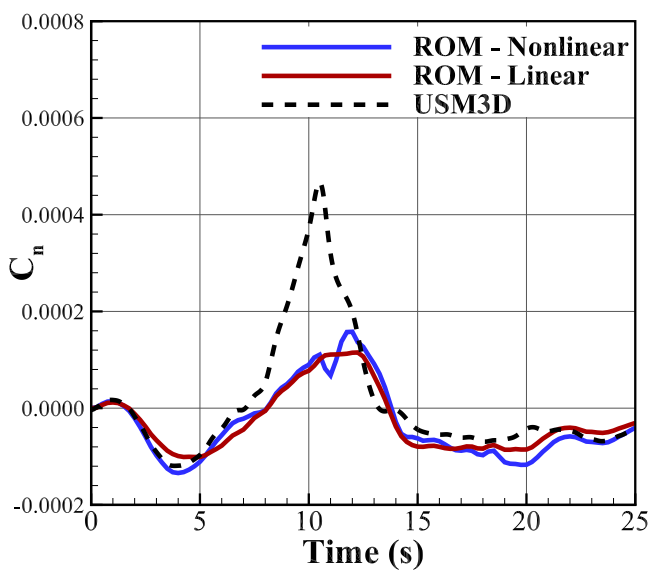

(d) Yawing Moment

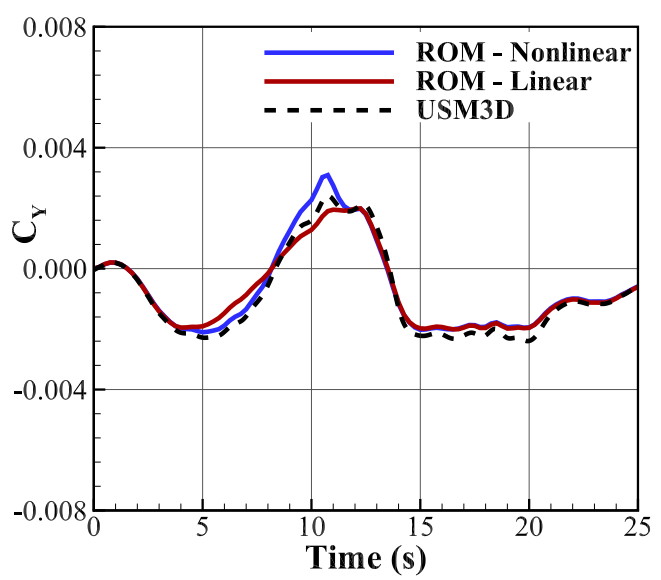

(e) Side Force

Fig. 18 Comparison of ROM and USM3D results for SACCON Immelmann maneuver. 10Mc grid. $M_{\infty}=0.2789, R_{c r e f}=1.57 \times 10^{6}$. 


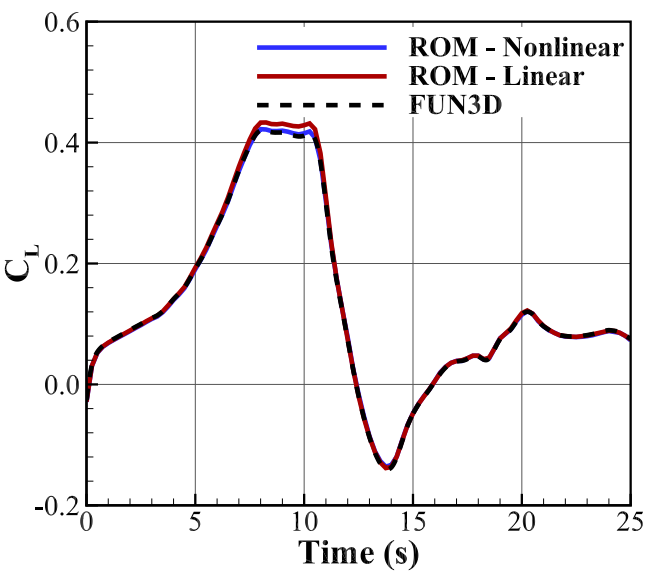

(a) Lift

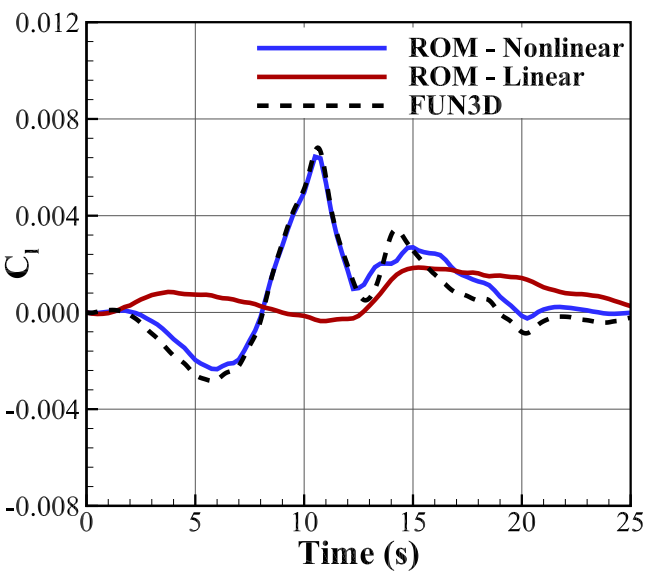

(c) Rolling Moment

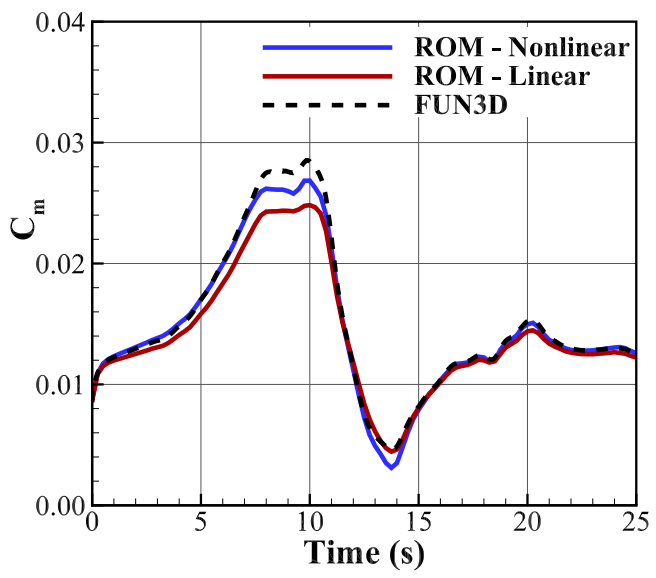

(b) Pitching Moment

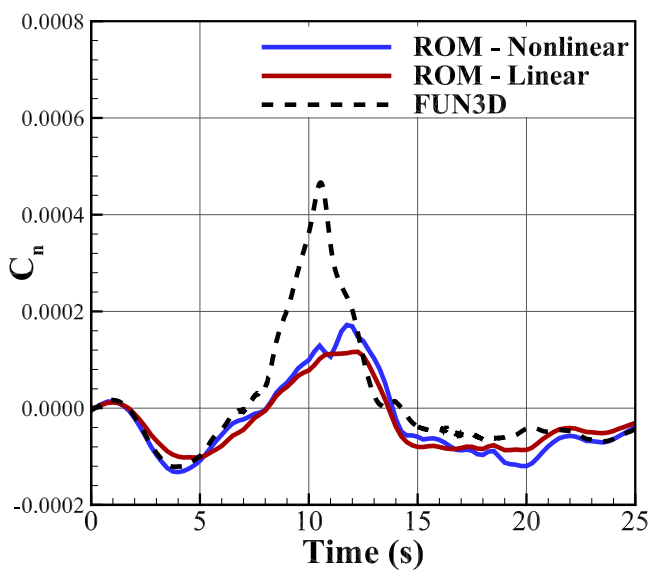

(d) Yawing Moment

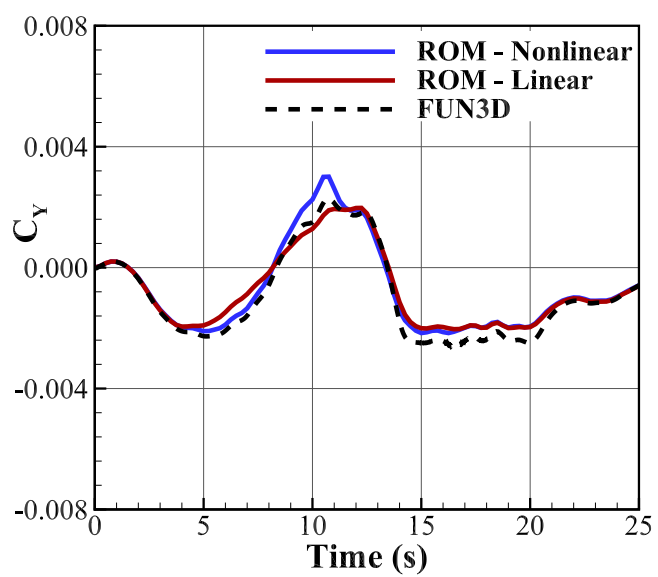

(e) Side Force

Fig. 19 Comparison of ROM and FUN3D results for SACCON Immelmann maneuver. 10Mn grid. $M_{\infty}=0.2789, R_{c r e f}=1.57 \times 10^{6}$. 


\section{Cost/benefit assessment of ROM}

The computational resources required for creating the SACCON indicial response solutions, the eight forced oscillation cases, and the two generalized flight maneuvers on the 10-million element grids are included in Table 6 The USM3D indicial solutions were computed on the K3-cluster Sandy Bridge nodes, consisting of Intel Xeon E5-2670 processors. The FUN3D indicial solutions were computed on the K4-cluster, consisting of Intel Gold 6148 Skylake processors. Both USM3D and FUN3D full-order maneuver solutions were computed on the NASA Advanced Supercomputing (NAS) facility's Pleiades supercomputer Ivy Bridge nodes, consisting of Intel Xeon E5-2680v2 processors. ROM computational times are given as computed using MATLAB computations performed on a $2 \mathrm{GHz}$ Intel Core i7 MacBook Pro personal laptop.

Table 6 CPU hours required to create ROM and flight maneuver solutions with grids 10Mc and 10Mn.

\begin{tabular}{|c|c|c|c|c|c|c|c|}
\hline & $\begin{array}{c}\text { Static } \\
\text { Initialization }\end{array}$ & $\begin{array}{l}\text { Indicial } \\
\text { Steps }\end{array}$ & Total & $\begin{array}{r}\text { Half } \\
(18\end{array}$ & $\begin{array}{l}\text { azy-8 } \\
\text { ec.) }\end{array}$ & $\begin{array}{l}\text { Immelmann } \\
(25 \mathrm{sec} .)\end{array}$ & $\begin{array}{l}\text { Forced Oscillations } \\
\text { (8 cases, } 2 \text { cycles) }\end{array}$ \\
\hline USM3D & 5,884 & 8,456 & 14,340 & 43,317 & 7,988 & 12,317 & 59,464 \\
\hline FUN3D & 21,679 & 23,015 & 44,694 & - & 24,268 & 34,915 & 74,116 \\
\hline ROM - Linear & - & - & - & - & 0.01 & 0.02 & 0.00 - Avg. \\
\hline ROM - Nonlinear & - & - & - & - & 0.32 & 1.19 & 0.01 - Avg. \\
\hline$\Delta t^{*}$ & & 0.01 & 0.01 & 0.01 & 0.10 & 0.10 & 0.01 \\
\hline
\end{tabular}

A full set of 75 indicial responses was computed from each flow solver with a temporal resolution of $\Delta t^{*}=0.01$. The indicial step responses were restarted from converged steady-state solutions at the appropriate initial static conditions. As observed in Table 6 the static initialization contributes a considerable portion toward the total cost of creating the ROM input. The combined total computational cost to generate the ROM input data with USM3D and FUN3D was 14,340 and 44,694 CPU hours, respectively. For a relative cost comparison at the same temporal resolution of $\Delta t^{*}=0.01$, a full USM3D simulation was flown over the 18-second half Lazy-8 maneuver and required 41,317 CPU hours. Thus, the indicial response ROM was created using 33-percent less CPU resources than a full USM3D simulation flown over an 18-second maneuver. As noted in the discussion of Fig. 13 in Section VI.D.1, the ROM captures the impulse flow transients to a level of temporal accuracy of a comparable CFD simulation.

A further cost comparison can be made in Table 6 with the 8 Forced Oscillation cases that were computed with $\Delta t^{*}=0.01$. USM3D and FUN3D required 59,464 and 74,116 CPU hours, respectively. The computational resources required to generate full CFD simulations for the half Lazy- 8 maneuver and Immelmann turn maneuver with an order-of-magnitude larger time step of $\Delta t^{*}=0.1$ are 7,988 and 12,317 CPU hours with USM3D, respectively, and 24,268 and 34,915 CPU hours with FUN3D, respectively.

The costs associated with the ROM are primarily attributed to the model generation process. Once the indicial responses are simulated, the resultant ROM can then be used to fly through any maneuver within the $\alpha, \beta$, and $M$ envelope of the response solutions in a matter of seconds or minutes (e.g., Immelmann turn, half Lazy-8, forced oscillations, wideband input, etc.). Note that the CPU hours needed to run the flight manuevers in Table 6 with the linear ROM is almost negligible. With the nonlinear ROM, all aerodynamic coefficients were generated for the half Lazy- 8 in 0.32 hours and the Immelmann turn in 1.19 hours. The utility in using such an approach for efficiently providing unsteady aerodynamic CFD predictions for unconventional vehicle configurations is evident, where the computational benefits scale with the number of evaluations needed. 


\section{Future Work}

\section{A. Efficient System Identification}

The recent focus vehicle for the Technologies for Airplane State Awareness subproject has been a Generic T-Tail (GTT), aft twin-engine commuter jet. Considerable effort [8, 10, 11, 43] has been directed toward bringing aerodynamic data from CFD sources into the system identification (SysID) process for constructing dynamic stability derivatives for transport aircraft. The most recent attempt is reported in Refs. [7, 8] where the resultant derivatives appear reasonable, but the required computational resources render it impractical for populating large databases.

An alternate approach is proposed in Fig. 20 where a high-fidelity surrogate-based indicial reduced-order model is created for the GTT as previously described. Since the ROM captures the transient behavior of motions to the fidelity of a comparable CFD solution, and the surrogate framework enables coverage of a broad flight envelope, then the ROM can be used to very quickly generate a large matrix of responses to typical SysID specialty inputs across the envelope. The classic SysID approaches are now able to capture any transients or nonlinearities in the stability derivatives. Furthermore, the aerodynamic data can be computed at flight Reynolds numbers and Mach numbers to provide a more representative model.

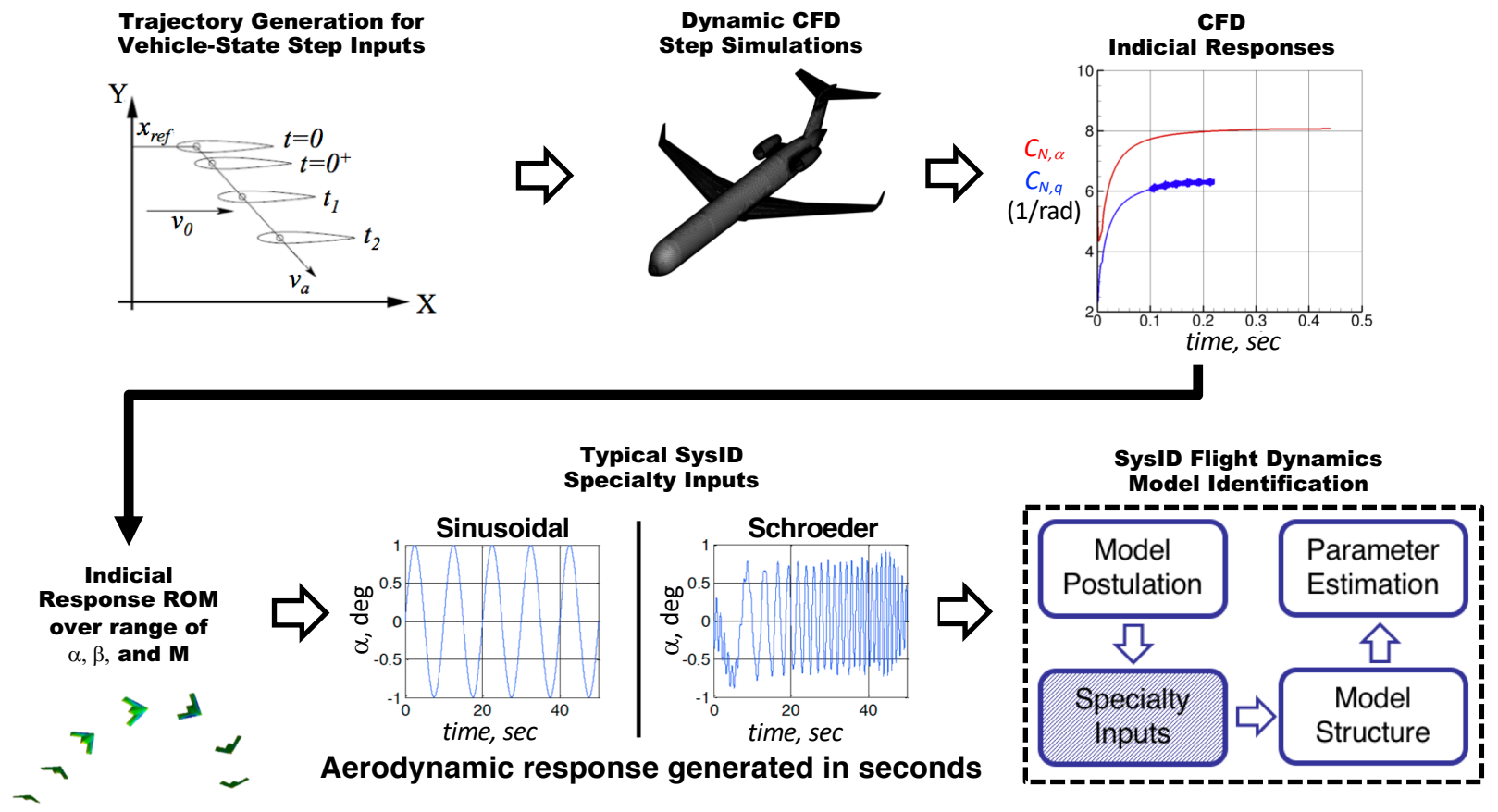

Fig. 20 Proposed use of ROM for more efficient System Identification process.

An initial attempt was made to create such a model for the GTT as depicted in Fig. 21. Unfortunately, the wing exhibits a very unsteady stall behavior between angles of attack of $8^{\circ}$ and $14^{\circ}$. Since the indicial response theory requires the step responses to settle toward an asymptotic steady state, an approach is proposed to modify the unsteady responses during stall in an attempt to capture the overall lag and sluggesh behavior generally experienced during a stall. Figure 21 illustrates a smooth fitting of the unsteady data that is ultimately faired to a steady state. With this modification, an indicial ROM may be able to provide the dominant sensory queues to the pilot during stall, such as the long time lags and growing instability of pitch between angles of attack $10^{\circ}$ and $14^{\circ}$. 


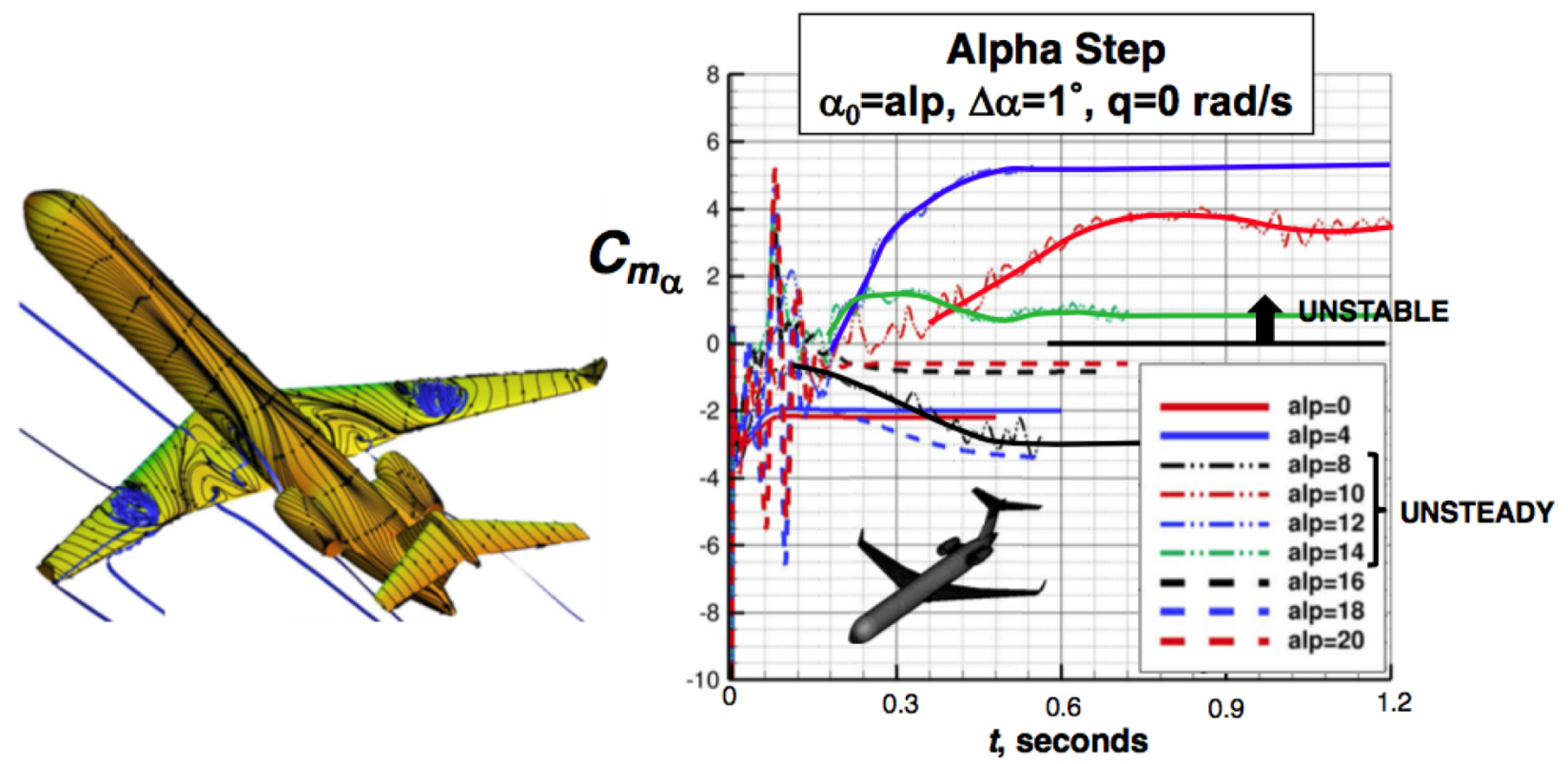

Fig. 21 Proposed approach for creating GTT stall ROM to capture general stall characteristics. Left image depicts stalled flow at $\alpha=12^{\circ}$.

\section{B. Efficient Aeroelastic Effects}

Driven by the pursuit of unconventional configurations and increasingly flexible structures, there is a growing need for modeling the nonlinear, unsteady aerodynamic flow phenomena common to present operational flight envelopes. While notable progress has been made in unsteady aerodynamics reduced-order modeling, a significant gap exists in the development of multidisciplinary ROMs for predicting the aerodynamic performance in consideration of aeroelasticity effects. Prior aeroelastic ROM research has been largely confined to the study of aeroelastic instability phenomena and remains limited for flight performance assessments. In order to advance the field of reduced-order modeling and enable relevant, practical virtual flight predictions, it is imperative to develop and/or extend reduced-order modeling methodologies to capture the multidisciplinary interactions present in modern flexible aircraft.

The second author is pursuing a PhD topic titled "An Unsteady Aerodynamics Reduced-Order Modeling Method for Maneuvering, Flexible Flight Vehicles," which aims to explore the benefits of a surrogate-based, aeroelastic indicial response ROM. In this approach, aeroelastic indicial responses are simulated via prescribed rigid body motions, which isolate the effects of angle of attack and sideslip angle from angular rates, while fluid-structure interactions are captured at the subiterative level through coupling to a linear modal structural solver. As done in the present work, a nonlinear extension of indicial response theory is demonstrated through application of kriging surrogate modeling to a database of locally linear step responses, and models are created by applying the mathematical principle of convolution to interpolated aeroelastic indicial responses.

The validity of traditional reduced-order modeling assumptions is evaluated for use in flexible vehicle modeling. It is anticipated that the vehicle-state motion parameter dependencies and sampling requirements will change for more complex configurations. Additionally, indicial responses for positive and negative steps in angles of attack are typically assumed to be symmetric. However, as shown in the present work, asymmetric loading responses can be encountered for unconventional configurations. Because flexible vehicles are often designed with asymmetric stiffness properties, it is likely that more pronounced asymmetric loading responses will be observed relative to rigid vehicles. Furthermore, the impact of changes in the type and number of structural modes included in the modal structural analysis on the aeroelastic ROM predictions will be investigated. Results are to be presented for the N+2 low-boom supersonic configuration undergoing a series of harmonic forced oscillations and the X-56A aircraft undergoing turn maneuvers representative of flight tests. The efficient reduced-order modeling solutions could provide a practical option for evaluating the vehicle dynamics of maneuvering, flexible vehicles using high-fidelity simulations. 


\section{Summary}

The Ghoreyshi surrogate-based, indicial response reduced-order model has been evaluated using two NASA unstructured flow solvers, USM3D and FUN3D, as a possible means to efficiently augment civil aircraft motion-based flight training simulators with high-fidelity aerodynamic data. The dynamic stability terms within typical flight simulator models are based on common S\&C damping derivatives developed by measuring the in-phase and out-of-phase responses from sinusoidal forced oscillations on wind-tunnel models tested at very low Reynolds and Mach numbers. A high-fidelity CFD-based reduced-order model offers the potential to quickly augment such data with flight-scaled damping derivatives.

The candidate ROM methodology attempts to match the nonlinear predictive capability of full CFD simulations at a fraction of the computational cost. This approach requires that indicial step functions be computed through prescribed grid motion trajectories that directly capture the indicial responses (no training maneuvers), which is in contrast to the classic approach of identifying indicial functions using assumed parametric model and training maneuvers through SysID applied to CFD data.

Prior to creating the indicial response data for the tailless SACCON configuration, sensitivities of the flow solutions to grid density, time step, and subiteration were assessed on an 18-second half Lazy-8 flight maneuver. From this, a solution strategy was chosen for computing indicial response solutions with each code. Once the 75 indicial responses were generated, the ROM was exercised for several sinusoidal forced oscillation conditions about the pitch, roll, and yaw axis. The result was a very good correlation with the moment hysteresis loops from full CFD solutions, even for the sensitive yawing moments that are extremely small in magnitude for the tailless aircraft. Furthermore, the impulse transients from the sudden start of motion were captured almost exactly by the ROM, thus demonstrating that the transient behavior of a full CFD solution can be resolved to the level of time discretization of the indicial response solution.

A second evaluation was performed by correlating the longitudinal and lateral coefficients from the ROM and full-order CFD solutions for the SACCON flown through the half Lazy-8 and Immelmann turn maneuvers. General agreement was found between the ROM and full-order CFD solutions with the exception of the yawing moment coefficient, where the results suggest modifying the ROM assumptions to include a nonlinear dependency on angle of attack for the angular rates of rotation. Furthermore, the results confirmed a sensitivity in moment coefficient predictions for Mach numbers approaching the limits of compressible flow. Despite these observations, reasonable prediction results were obtained at a computational cost significantly less than what was required for full-order CFD maneuver simulations. The demonstrated utility of the surrogate-based indicial response approach warrants consideration for future applications in providing S\&C predictions for commercial aircraft at stall conditions and for maneuvering, flexible vehicles.

\section{Acknowledgments}

The timely assistance of Dr. Mohagna J. Pandya of NASA Langley Research Center and Dr. Boris Diskin of the National Institute of Aerospace is greatly appreciated for implementing the generalized moving grid capability into the new hybrid cell USM3D flow solver and then working closely with the first author to establish efficient application guidelines and practices. Additionally, the authors thank Mr. Bob Biedron of NASA Langley Research Center for his assistance in implementing the FUN3D flow solver for the generalized moving grid simulations.

The authors greatly appreciate the many conversations with Dr. Medhi Ghoreyshi from the U.S. Air Force Academy, his openness to share his vast knowledge and experience with the indicial-based reduced-order model, and his sharing the flight trajectories for the half Lazy-8 and Immelmann turn maneuvers.

This work was sponsored by the SE209 Flight Simulation element of the Technologies for Airplane State Awareness subproject within the System Wide Safety Project under the NASA Airspace Operations and Safety Program. Their support is gratefully acknowledged. 


\section{References}

[1] Belcastro, C., and Foster, J., “Aircraft loss-of-control accident analysis,” AIAA Paper 2010-8004, 2010.

[2] Shah, G. H., Cunningham, K., Foster, J. V., Fremaux, C. M., Stewart, E. C., Wilborn, J. E., Gato, W., and Pratt, D. W., "Wind-tunnel investigation of commercial transport aircraft aerodynamics at extreme flight conditions," Tech. rep., SAE Technical Paper, 2002.

[3] Foster, J., Cunningham, K., Fremaux, C., Shah, G., Stewart, E., Rivers, R., Wilborn, J., and Gato, W., "Dynamics modeling and simulation of large transport airplanes in upset conditions," AIAA Paper 2005-5933, 2005.

[4] Wilborn, J., and Foster, J., "Defining commercial transport loss-of-control: A quantitative approach," AIAA Paper 2004-4811, 2004.

[5] "Qualification, Service, and Use of Crewmembers and Aircraft Dispatchers; Final Rule, 14 CFR Part 121," Federal Aviation Administration, Federal Register, Vol 78, No. 218, 2013.

[6] Frink, N. T., Murphy, P. C., Atkins, H. L., Viken, S. A., Petrilli, J. L., Gopalarathnam, A., and Paul, R. C., "Computational Aerodynamic Modeling Tools for Aircraft Loss of Control," Journal of Guidance, Control, and Dynamics, Vol. 40, No. 4, 2016, pp. 789-803.

[7] McMillin, S. N., Frink, N. T., Murphy, P. C., Cunningham, K., and Shah, G. H., "Computational Study of a Generic T-tail Transport," Paper to be presented at the 2019 AIAA Science and Technology Forum and Exposition, 2019.

[8] Murphy, P. C., Frink, N. T., McMillin, S. N., Cunningham, K., and Shah, G. H., "Unsteady Model Estimation for Generic T-Tail Transport Aircraft Using Computational Data," Paper to be presented at the 2019 AIAA Science and Technology Forum and Exposition, 2019.

[9] Murphy, P., Klein, V., Frink, N., and Vicroy, D., "System identification applied to dynamic CFD simulation and wind tunnel data," AIAA Paper 2011-6522, 2011.

[10] Murphy, P., Klein, V., and Frink, N., "Unsteady aerodynamic modeling in roll for the NASA generic transport model," AIAA Paper 2012-4652, 2012.

[11] Murphy, P. C., Klein, V., and Frink, N. T., "Nonlinear Unsteady Aerodynamic Modeling Using Wind-Tunnel and Computational Data," Journal of Aircraft, Vol. 54, No. 2, 2017, pp. 659-683.

[12] Dean, J., Morton, S., McDaniel, D., Clifton, J., and Bodkin, D., "Aircraft stability and control characteristics determined by system identification of CFD simulations," AIAA Paper 2010-6378, 2008.

[13] Morton, S. A., and McDaniel, D. R., "CFD-Based Stability and Control Modeling of Fighter Aircraft Useful to the GNC Community," AIAA Paper 2018-2994, 2018.

[14] Ghoreyshi, M., and Cummings, R. M., "Unsteady aerodynamics modeling for aircraft maneuvers: A new approach using time-dependent surrogate modeling," Aerospace Science and Technology, Vol. 39, 2014, pp. 222-242.

[15] Vicroy, D. D., Loeser, T. D., and Schütte, A., "Static and forced-oscillation tests of a generic unmanned combat air vehicle," Journal of Aircraft, Vol. 49, No. 6, 2012, pp. 1558-1583.

[16] Cummings, R. M., and Schütte, A., "Integrated computational/experimental approach to unmanned combat air vehicle stability and control estimation," Journal of Aircraft, Vol. 49, No. 6, 2012, pp. 1542-1557.

[17] Cummings, R. M., and Schütte, A., "The NATO STO Task Group AVT-201 on 'Extended Assessment of Stability and Control Prediction Methods for NATO Air Vehicles'," AIAA Paper 2014-2000, 2014.

[18] Frink, N. T., “Tetrahedral unstructured Navier-Stokes method for turbulent flows," AIAA journal, Vol. 36, No. 11, 1998, pp. $1975-1982$.

[19] Pandya, M. J., Frink, N. T., Ding, E., and Parlette, E., “Toward Verification of USM3D Extensions for Mixed Element Grids," AIAA Paper 2013-2541, 2013.

[20] Pandya, M. J., Diskin, B., Thomas, J. L., and Frink, N. T., "Improved Convergence and Robustness of USM3D Solutions on Mixed-Element Grids," AIAA Journal, 2016, pp. 2589-2610.

[21] Pandya, M. J., Diskin, B., Thomas, J. L., and Frink, N. T., “Assessment of USM3D Hierarchical Adaptive Nonlinear Method Preconditioners for Three-Dimensional Cases,” AIAA Journal, 2017, pp. 3409-3424. 
[22] Pandya, M. J., Jespersen, D. C., Diskin, B., and Thomas, J. L., "Accuracy, Scalability and Efficiency of Mixed-Element USM3D for Benchmark Three-Dimensional Flows," Paper to be presented at the 2019 AIAA Science and Technology Forum and Exposition, 2019.

[23] Allmaras, S. R., and Johnson, F. T., "Modifications and clarifications for the implementation of the Spalart-Allmaras turbulence model," Seventh international conference on computational fluid dynamics (ICCFD7), 2012, pp. 1-11.

[24] Biedron, R. T., Carlson, J.-R., Derlaga, J. M., Gnoffo, P. A., Hammond, D. P., Jones, W. T., Kleb, B., Lee-Rausch, E. M., Nielsen, E. J., Park, M. A., et al., "FUN3D Manual: 13.3,” 2018.

[25] Biedron, R., and Thomas, J., "Recent enhancements to the FUN3D flow solver for moving-mesh applications," AIAA Paper 2009-1360, 2009.

[26] “HeldeMmesh User Manual, Version 3.02,” 2018.

[27] Frink, N. T., Pirzadeh, S. Z., Parikh, P. C., Pandya, M. J., and Bhat, M., "The NASA tetrahedral unstructured software system (TetrUSS)," The Aeronautical Journal, Vol. 104, No. 1040, 2000, pp. 491-499.

[28] Pandya, M., Frink, N. T., Abdol-Hamid, K. S., Samareh, J. A., Parlette, E. B., and Taft, J. R., "Enhancements to TetrUSS for NASA Constellation Program,” Journal of Spacecraft and Rockets, Vol. 49, No. 4, 2012, pp. 617-631.

[29] Pirzadeh, S. Z., "Advanced unstructured grid generation for complex aerodynamic applications," AIAA journal, Vol. 48, No. 5, 2010, pp. 904-915.

[30] Tobak, M., Chapman, G. T., and Schiff, L. B., "Mathematical modeling of the aerodynamic characteristics in flight dynamics," Proceedings of the Berkeley-Ames Conference on Nonlinear Problems in Control and Fluid Dynamics, Vol. 2, Math Science Pr, 1984, p. 435.

[31] Tobak, M., and Chapman, G. T., "Nonlinear problems in flight dynamics involving aerodynamic bifurcations," 1985.

[32] Reisenthel, P. H., "Development of a nonlinear indicial model using response functions generated by a neural network (in order to predict dynamics of maneuvering aircraft and missiles)," 1997.

[33] Reisenthel, P. H., and Bettencourt, M. T., "Data-based aerodynamic modeling using nonlinear indicial theory," AIAA paper, 1999, pp. 99-0763.

[34] Ghoreyshi, M., Jirásek, A., and Cummings, R. M., "Computational investigation into the use of response functions for aerodynamic-load modeling," AIAA journal, Vol. 50, No. 6, 2012, pp. 1314-1327.

[35] Ghoreyshi, M., and Cummings, R., "Aerodynamics modeling of a maneuvering aircraft using indicial functions," 50th AIAA Aerospace Sciences Meeting including the New Horizons Forum and Aerospace Exposition, 2012, p. 689.

[36] Ghoreyshi, M., Cummings, R. M., and Lofthouse, A., "Sampling Strategies for Reduced-Order Modeling of Nonlinear and Unsteady Aerodynamics," 52nd Aerospace Sciences Meeting, 2014, p. 0047.

[37] Skujins, T., and Cesnik, C. E., "Reduced-Order Modeling of Unsteady Aerodynamics Across Multiple Mach Regimes," Journal of Aircraft, Vol. 51, No. 6, 2014, pp. 1681-1704.

[38] Lophaven, S. N., Nielsen, H. B., and Søndergaard, J., DACE: a Matlab kriging toolbox, Vol. 2, Citeseer, 2002.

[39] Schütte, A., Hummel, D., and Hitzel, S. M., "Flow physics analyses of a generic unmanned combat aerial vehicle configuration," Journal of Aircraft, Vol. 49, No. 6, 2012, pp. 1638-1651.

[40] Vallespin, D., Boelens, O., and Cummings, R., "Comparison of results for predicting highly non-linear vortical flow features of the UCAV SACCON configuration," NATO Research and Technology Organisation, Applied Vehicle Technology (AVT-161), Final Report, Chapter 16, 2012.

[41] Jirasek, A., Cummings, R. M., Schuette, A., and Huber, K. C., "The NATO STO AVT-201 Task Group on Extended assessment of Stability an Control Prediction Methods for NATO Air Vehicles: Summary, Conclusions and Lessons Learned," AIAA Paper 2014-2394, 2014.

[42] Frink, N., "Strategy for dynamic CFD simulations on SACCON configuration,” AIAA Paper 2010-4559, 2010.

[43] Murphy, P. C., Frink, N. T., McMillin, S. N., Cunningham, K., and Shah, G. H., "Efficient Unsteady Model Estimation Using Computational and Experimental Data," AIAA Paper 2018-3622, 2018. 


\section{Appendix A - Simulating Maneuvering Vehicle Trajectories}

\section{A. Coordinate Frames}

Figure 22 shows the SACCON configuration and the two primary axes systems: 1) the traditional flight mechanics reference frame (blue), and 2) the CFD reference frame (red) common to the USM3D and FUN3D flow solvers. The CFD reference frame differs from the traditional flight mechanics reference frame by a 180 degree rotation about the $y$ axis.

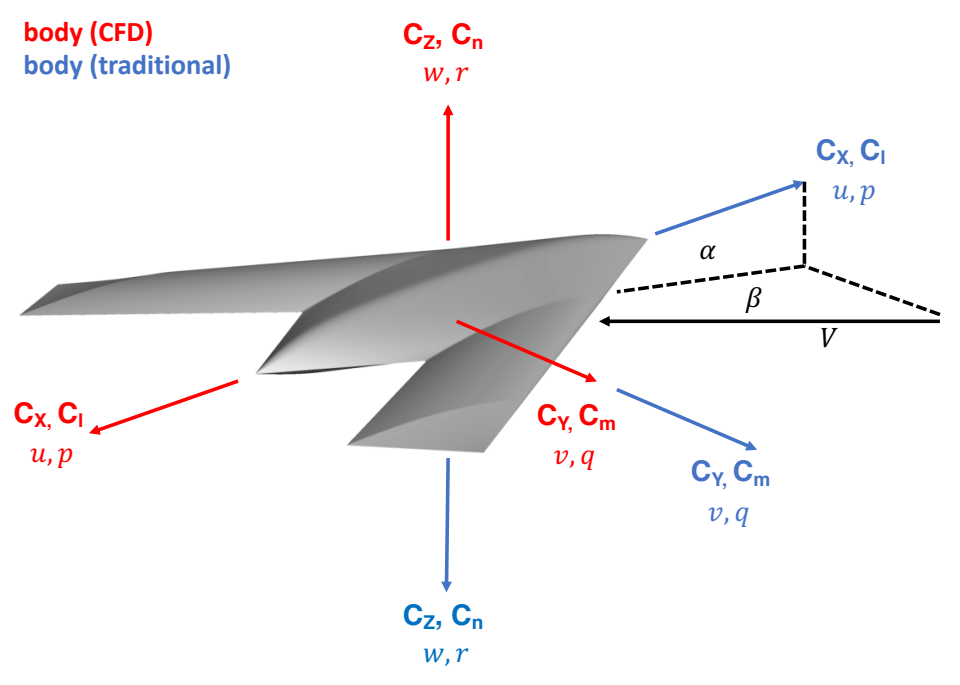

Fig. 22 Trajectory axes systems.

In the traditional flight reference frame, the aerodynamic force coefficients are:

- Axial force, $C_{X}>0$ along x-nose,

- Side force, $C_{Y}>0$ along y-right, and

- Normal force, $C_{Z}>0$ along z-down,

while in the CFD reference frame, the aerodynamic force coefficients are:

- Axial force, $C_{X}>0$ along x-tail,

- Side force, $C_{Y}>0$ along y-right, and

- Normal force, $C_{Z}>0$ along z-up.

The aerodynamic moment coefficients are defined to be positive according to the right-hand rule for the respective coordinate systems. In the traditional flight reference frame, the aerodynamic moments coefficients are:

- Rolling moment, $C_{l}>0$ with right-wing-down,

- Pitching moment, $C_{m}>0$ with nose-up, and

- Yawing moment, $C_{n}>0$ with nose-right,

while in the CFD reference frame, the aerodynamic moment coefficients are:

- Rolling moment, $C_{l}>0$ with right-wing-up,

- Pitching moment, $C_{m}>0$ with nose-up, and

- Yawing moment, $C_{n}>0$ with nose-left. 


\section{B. Trajectory Transformation}

Vehicle flight trajectories are defined by a wind velocity $(\alpha, \beta, M)$ and orientation $(\phi, \theta, \psi)$ at each point in time. The initial assumption for the trajectory transformation is that the grid orientation at time zero aligns with the inertial or horizontal axis, i.e., at $t=0$, then $\phi=\theta=\psi=0$. Furthermore, at $t=0$ the $V_{0}$ is the freestream velocity in the wind axis, and $\alpha_{0}, \beta_{0}$ are the angle of attack and sideslip angle at time zero. When computing the trajectories for USM3D and FUN3D, substitute freestream $M_{0}$ for $V_{0}$ and $M(t)$ for $V(t)$ in the following.

The initial flow conditions at $t=0$ are used to establish a reference velocity for the grid motion. Assuming $\phi=\theta=\psi=0$, the components of the reference velocity are defined by Eq. 12 .

$$
\left\{\begin{array}{c}
u_{\text {ref }} \\
v_{\text {ref }} \\
w_{\text {ref }}
\end{array}\right\}=V_{0}\left\{\begin{array}{c}
\cos \left(\alpha_{0}\right) \cos \left(\beta_{0}\right) \\
\sin \left(\beta_{0}\right) \\
\sin \left(\alpha_{0}\right) \cos \left(\beta_{0}\right)
\end{array}\right\}
$$

Next tranform the prescribe time dependent $\alpha, \beta$ and $V$ from the wind axis to body axis, using Eq. 13

$$
\left\{\begin{array}{l}
u_{b}(t) \\
v_{b}(t) \\
w_{b}(t)
\end{array}\right\}=V(t)\left\{\begin{array}{c}
\cos (\alpha(t)) \cos (\beta(t)) \\
\sin (\beta(t)) \\
\sin (\alpha(t)) \cos (\beta(t))
\end{array}\right\}
$$

Then transform through the Euler angles (roll, pitch, and yaw) from the body axis to the horizontal axis aligned with the inertial axes at time $t=0$ using Eqs. 14, 15, and 16 .

$$
\begin{gathered}
R(t)=R_{z}(\phi(t)) R_{y}(\theta(t)) R_{x}(\psi(t)) \\
R_{z}(\phi)=\left\{\begin{array}{ccc}
\cos \psi & -\sin \psi & 0 \\
\sin \psi & \cos \psi & 0 \\
0 & 0 & 1
\end{array}\right\}, R_{y}(\phi)=\left\{\begin{array}{ccc}
\cos \theta & 0 & \sin \theta \\
0 & 1 & 0 \\
-\sin \theta & 0 & \cos \theta
\end{array}\right\}, R_{x}(\phi)=\left\{\begin{array}{ccc}
1 & 0 & 0 \\
0 & \cos \phi & -\sin \phi \\
0 & \sin \phi & \cos \phi
\end{array}\right\} \\
\left\{\begin{array}{l}
u_{h}(t) \\
v_{h}(t) \\
w_{h}(t)
\end{array}\right\}=R(t)\left\{\begin{array}{l}
u_{b}(t) \\
v_{b}(t) \\
w_{b}(t)
\end{array}\right\}
\end{gathered}
$$

The final grid velocity is computed by subtracting the reference velocity, Eq. 12, from the horizontal axis velocity components, Eq. 16, as described in Eq. 17. Note the sign changes that correct for the 180 degree rotation about the y-axis between the flight and CFD reference systems in Fig. 22

$$
\left\{\begin{array}{c}
U_{h}(t) \\
V_{h}(t) \\
W_{h}(t)
\end{array}\right\}=\left\{\begin{array}{c}
-u_{h}(t) \\
v_{h}(t) \\
-w_{h}(t)
\end{array}\right\}-\left\{\begin{array}{c}
-u_{r e f} \\
v_{\text {ref }} \\
-w_{\text {ref }}
\end{array}\right\}
$$

Finally, the change in translation at each time step in the CFD coordinate system is calculated as the change in position due to the average translation velocity between two successive time steps, as shown in Eq. 18 Once the translation is calculated, the position is calculated by the sum of the initial CG position and the translation vector, as shown in Eq. 19

$$
\begin{gathered}
\left\{\begin{array}{l}
T_{x}\left(t_{i+1}\right) \\
T_{y}\left(t_{i+1}\right) \\
T_{z}\left(t_{i+1}\right)
\end{array}\right\}=\left\{\begin{array}{l}
T_{x}\left(t_{i}\right) \\
T_{y}\left(t_{i}\right) \\
T_{z}\left(t_{i}\right)
\end{array}\right\}+\Delta t\left\{\begin{array}{l}
\frac{U_{h}\left(t_{i+1}\right)+U_{h}\left(t_{i}\right)}{2 a_{0}} \\
\frac{V_{h}\left(t_{i+1}\right) V_{h}\left(t_{i}\right)}{2 a_{0}\left(t_{i+1}\right)+W_{h}\left(t_{i}\right)} \\
\frac{W_{0}}{2 a_{0}}
\end{array}\right\} \\
\left\{\begin{array}{l}
C G_{x}(t) \\
C G_{y}(t) \\
C G_{z}(t)
\end{array}\right\}=\left\{\begin{array}{l}
C G_{x}\left(t_{0}\right) \\
C G_{y}\left(t_{0}\right) \\
C G_{z}\left(t_{0}\right)
\end{array}\right\}+\left\{\begin{array}{l}
T_{x}(t) \\
T_{y}(t) \\
T_{z}(t)
\end{array}\right\}
\end{gathered}
$$




\section{USM3D Motion File Generation}

USM3D motion files are defined by time, position $(X, Y, Z)$, and orientation $\left(R_{X}, R_{Y}, R_{Z}\right)$, as shown by Table 7 . Time, $t$, is defined by a non-dimensional time, normalized by speed of sound and incremented by a user-prescribed time step. Beginning from a static initialized solution, the body reference frame is assumed to align with the inertial reference frame, and thus, the initial position and orientation are zero. USM3D implicitly assumes the position is referenced to the vehicle CG, and the time-dependent position values are consistent with the translation values calculated in Eq. 18 The orientation is defined directly by the prescribed Euler angles, with roll and yaw defined to be negative to account for the difference between the CFD and flight trajectory reference frames.

Table 7 Example format of USM3D rigid body motion file.

\begin{tabular}{ccccccc}
\hline \hline$T_{U S M 3 D}$ & $X$ & $Y$ & $Z$ & $R_{X}$ & $R_{Y}$ & $R_{Z}$ \\
\hline$t_{0}$ & 0 & 0 & 0 & 0 & 0 & 0 \\
$t_{0}+\Delta t$ & $T_{X}\left(t_{1}\right)$ & $T_{Y}\left(t_{1}\right)$ & $T_{Z}\left(t_{1}\right)$ & $-\phi\left(t_{1}\right)$ & $\theta\left(t_{1}\right)$ & $-\psi\left(t_{1}\right)$ \\
$\vdots$ & $\vdots$ & $\vdots$ & $\vdots$ & $\vdots$ & $\vdots$ & $\vdots$ \\
$t_{0}+n \Delta t$ & $T_{X}\left(t_{n}\right)$ & $T_{Y}\left(t_{n}\right)$ & $T_{Z}\left(t_{n}\right)$ & $-\phi\left(t_{n}\right)$ & $\theta\left(t_{n}\right)$ & $-\psi\left(t_{n}\right)$ \\
\hline \hline
\end{tabular}

\section{FUN3D Motion File Generation}

FUN3D defines all rigid motions via application of $4 \times 4$ matrices to described affine transformations [24]. The $4 \times$ 4 transformation matrix contains both translation and orthonormal rotation components, as shown in Eq. 20, and maps a vehicle's initial position at time $t=0$, when aligned with the inertial reference frame, to its translated position at $t=t$.

$$
\left\{\begin{array}{l}
x \\
y \\
z \\
1
\end{array}\right\}=\left\{\begin{array}{cccc}
R_{11} & R_{12} & R_{13} & T_{x} \\
R_{21} & R_{22} & R_{23} & T_{y} \\
R_{31} & R_{32} & R_{33} & T_{z} \\
0 & 0 & 0 & 1
\end{array}\right\}\left\{\begin{array}{c}
x_{0} \\
y_{0} \\
z_{0} \\
1
\end{array}\right\}
$$

For maneuvering vehicle simulations, FUN3D maps from the fixed reference frame to the moving body coordinate frame through the sequence of "Origin to CG - Rotate about CG - CG to Origin - Translate" as shown by Eq. 21.

$$
[T(t)]=\left[T_{C G}\right]\left[R_{C F D}(t)\right]\left[T_{C G}\right]^{-1}+\left[T_{\text {grid }}(t)\right]
$$

The pure translation to the vehicle's CG location consists of a transform matrix defined by a $3 \times 3$ rotation submatrix consisting of an identity matrix with the final column accounting for the pure translation to the CG position coordinates from the inertial frame axes origin, as shown in Eq. 22. The rotation matrix consists of a composite rotation matrix calculated via chained rotation matrices from the flight trajectory Euler angles, as shown in Eq. 23. Note that the roll and yaw angles are defined to be negative to account for the change between the traditional reference frame and CFD reference frame. The translation matrix accounting for translation back to the origin is simply the inverse transform of the original translation matrix, as shown in Eq. 24. The second transform component is the pure grid translation component, which is calculated based on the time-dependent body velocity transformed into the inertial axes frame via Eq. 18.

$$
\begin{gathered}
{\left[T_{c g}\right]=\left\{\begin{array}{cccc}
1 & 0 & 0 & C G_{x}\left(t_{0}\right) \\
0 & 1 & 0 & C G_{y}\left(t_{0}\right) \\
0 & 0 & 1 & C G_{z}\left(t_{0}\right) \\
0 & 0 & 0 & 1
\end{array}\right\}} \\
{[R(t)]=\left[R_{z}(-\phi(t))\right]\left[R_{y}(\theta(t))\right]\left[R_{x}(-\psi(t))\right]}
\end{gathered}
$$




$$
\left[T_{\text {grid }}(t)\right]=\left\{\begin{array}{cccc}
0 & 0 & 0 & T_{x}(t) \\
0 & 0 & 0 & T_{y}(t) \\
0 & 0 & 0 & T_{z}(t) \\
0 & 0 & 0 & 1
\end{array}\right\}
$$

The FUN3D motion files defining the rigid body motion via transform matrices are formatted as outlined by Table 8 Starting from a static initialization, the vehicle CG location is defined relative to the origin with an identity matrix defining the coincident inertial and body reference frames. For successive iterations, the first line consists of a single entry defining the non-dimensional time, the second line consists of the updated CG location resulting from the transform matrix, which is then defined for the given iteration. The motion file should include the initial, intermediate, and final motion states for the trajectory. For a finer time discretization, FUN3D will interpolate between the motion file entries to update the vehicle's position and orientation.

Table 8 Example format of FUN3D rigid body motion file.

\begin{tabular}{cccc}
\hline \hline$t_{0}$ & & & \\
$C G_{X}\left(t_{0}\right)$ & $C G_{Y}\left(t_{0}\right)$ & $C G_{Z}\left(t_{0}\right)$ & \\
1 & 0 & 0 & 0 \\
0 & 1 & 0 & 0 \\
0 & 0 & 1 & 0 \\
0 & 0 & 0 & 1 \\
\hline$t_{0}+\Delta t$ & & & \\
$C G_{X}\left(t_{1}\right)$ & $C G_{Y}\left(t_{1}\right)$ & $C G_{Z}\left(t_{1}\right)$ & \\
$R_{11}\left(t_{1}\right)$ & $R_{12}\left(t_{1}\right)$ & $R_{13}\left(t_{1}\right)$ & $T_{x}\left(t_{1}\right)$ \\
$R_{21}\left(t_{1}\right)$ & $R_{22}\left(t_{1}\right)$ & $R_{23}\left(t_{1}\right)$ & $T_{y}\left(t_{1}\right)$ \\
$R_{31}\left(t_{1}\right)$ & $R_{32}\left(t_{1}\right)$ & $R_{33}\left(t_{1}\right)$ & $T_{z}\left(t_{1}\right)$ \\
0 & 0 & 0 & 1 \\
\hline$\vdots$ & $\vdots$ & $\vdots$ & $\vdots$ \\
\hline$t_{n}$ & & & \\
$C G_{X}\left(t_{n}\right)$ & $C G_{Y}\left(t_{n}\right)$ & $C G_{Z}\left(t_{n}\right)$ & \\
$R_{11}\left(t_{n}\right)$ & $R_{12}\left(t_{n}\right)$ & $R_{13}\left(t_{n}\right)$ & $T_{x}\left(t_{n}\right)$ \\
$R_{21}\left(t_{n}\right)$ & $R_{22}\left(t_{n}\right)$ & $R_{23}\left(t_{n}\right)$ & $T_{y}\left(t_{n}\right)$ \\
$R_{31}\left(t_{n}\right)$ & $R_{32}\left(t_{n}\right)$ & $R_{33}\left(t_{n}\right)$ & $T_{z}\left(t_{n}\right)$ \\
0 & 0 & 0 & 1 \\
\hline \hline
\end{tabular}




\section{Appendix B - Indicial Response Data}

This appendix contains the plots of indicial response data for the cases in Tables 3 and 4 All responses were computed to an $s_{\max }=10$ where asymptotic convergence is achieved. The abscissa on the following plots is compressed to illuminate the initial transients.

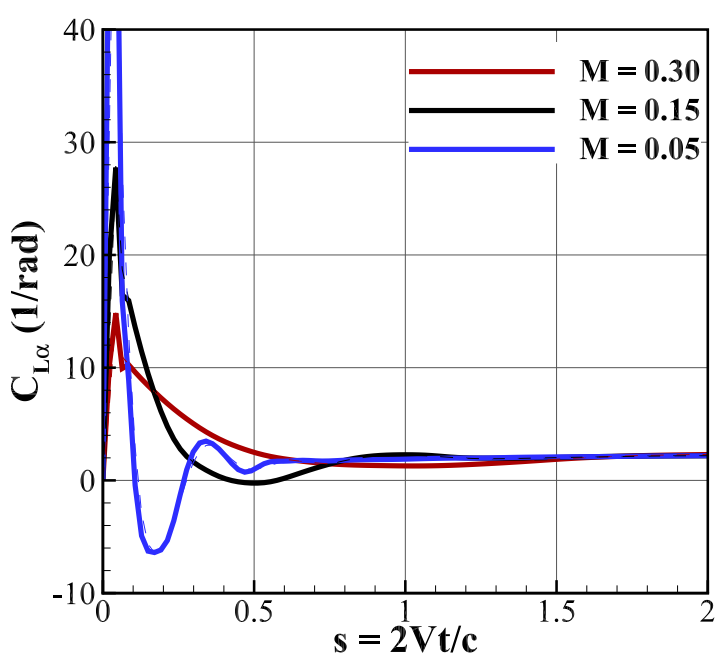

(a) linear $C_{L_{\alpha}}$ functions

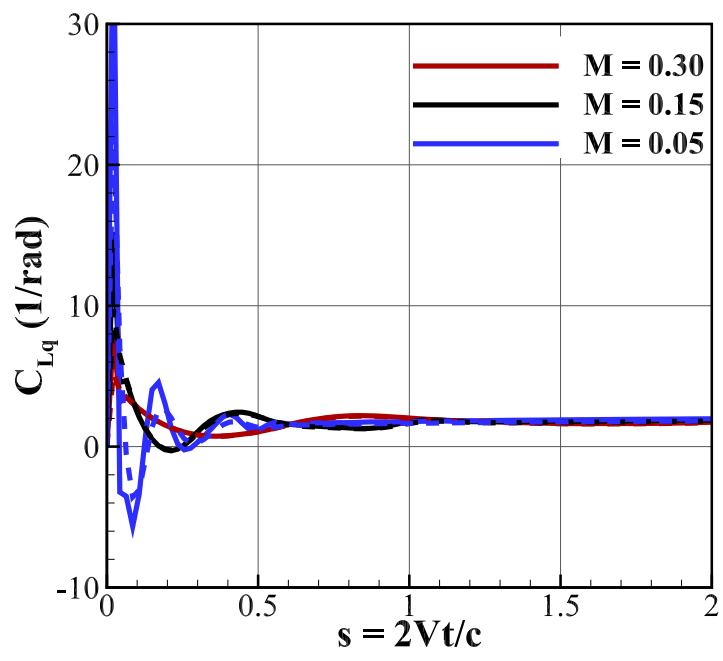

(c) linear $C_{L_{q}}$ functions

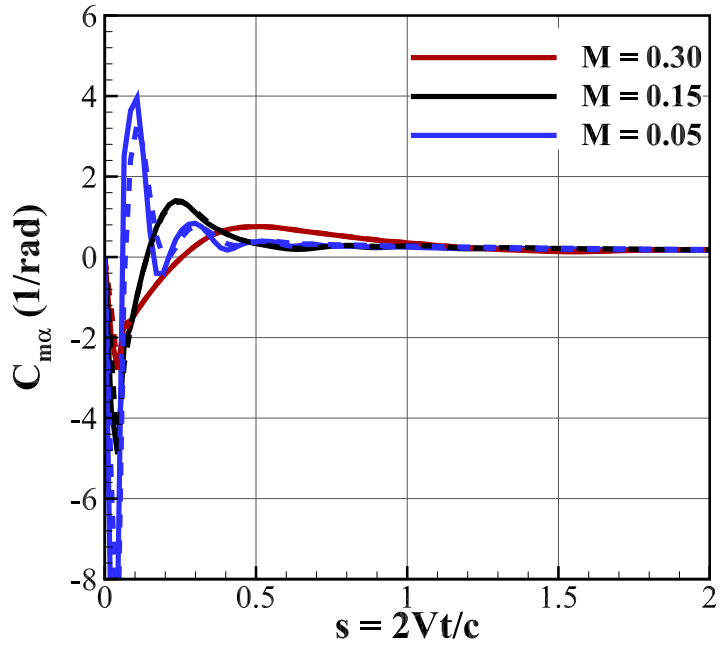

(b) linear $C_{m_{\alpha}}$ functions

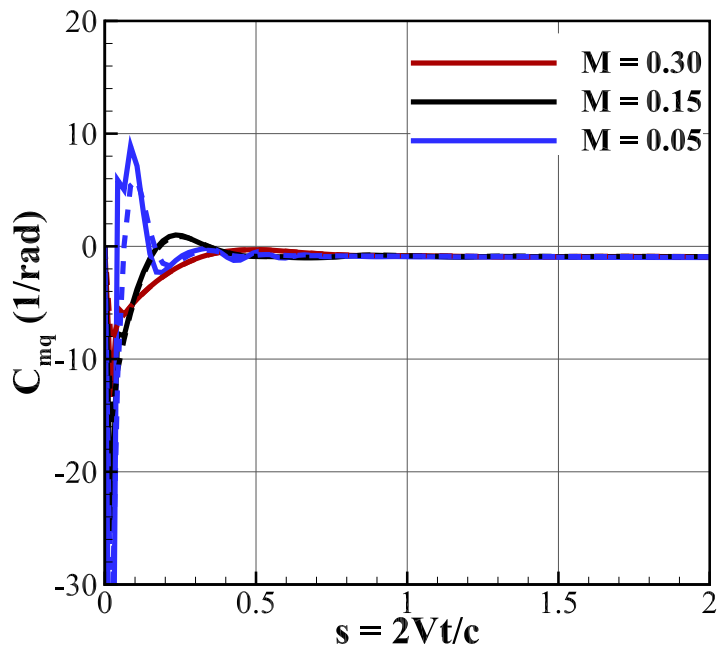

(d) linear $C_{m_{q}}$ functions

Fig. 23 The linear lift and pitching moment indicial functions with a unit step change of angle of attack (a-b) and normalized pitch rate (c-d). USM3D (dashed) and FUN3D (solid). 


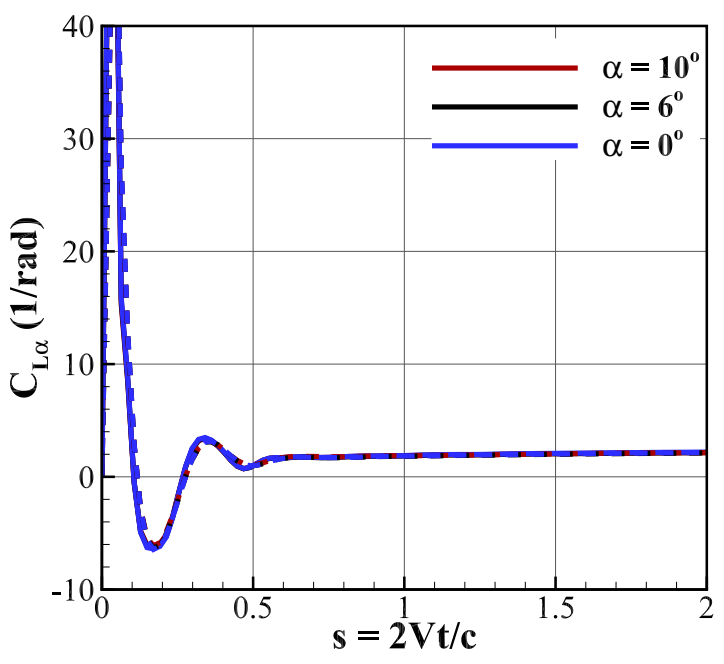

(a) nonlinear $C_{L_{\alpha}}$ functions ( $\left.M=0.05\right)$

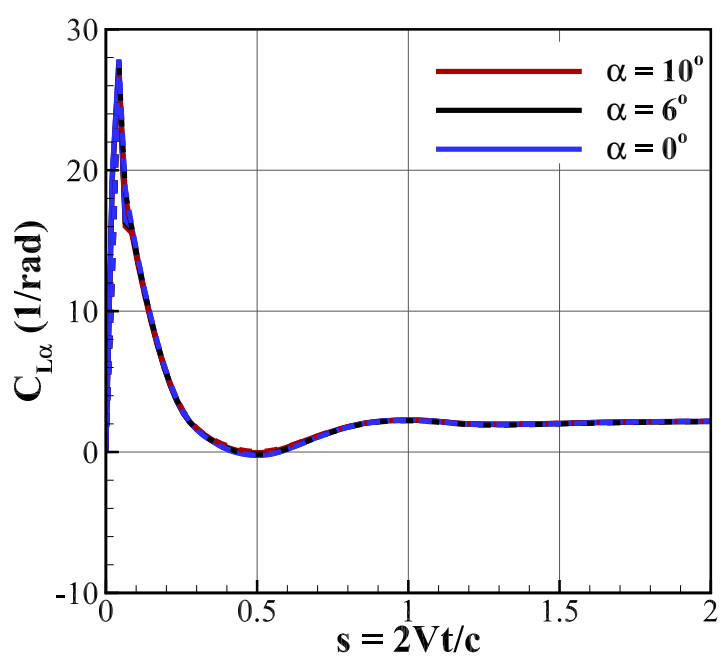

(c) nonlinear $C_{L_{\alpha}}$ functions ( $\left.M=0.15\right)$

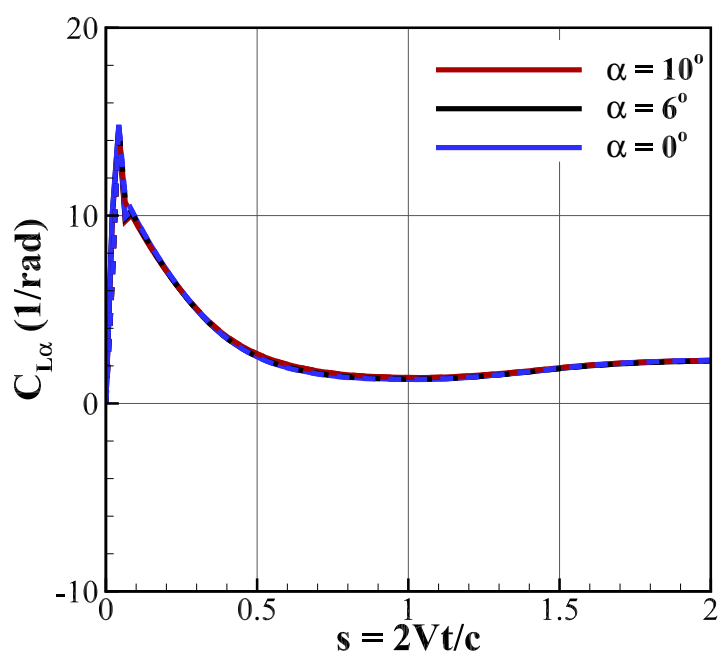

(e) nonlinear $C_{L_{\alpha}}$ functions ( $M=0.3$ )

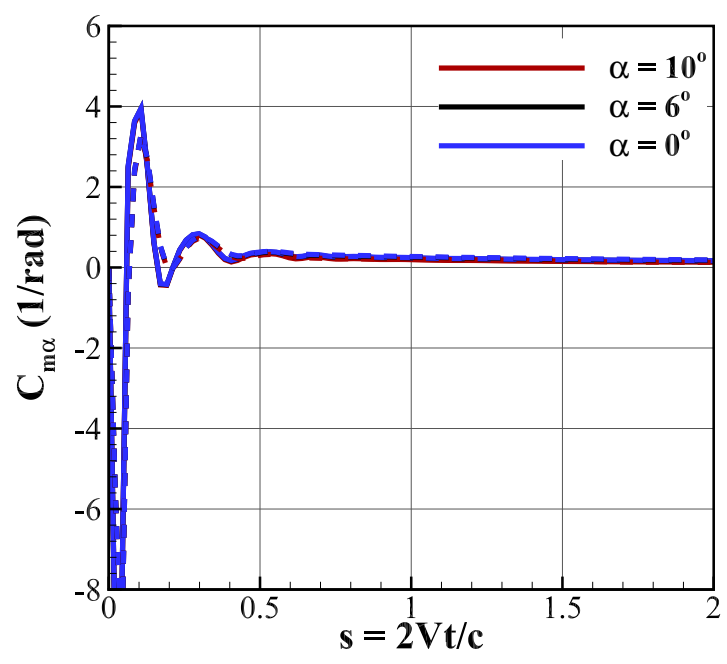

(b) nonlinear $C_{m_{\alpha}}$ functions ( $M=0.05$ )

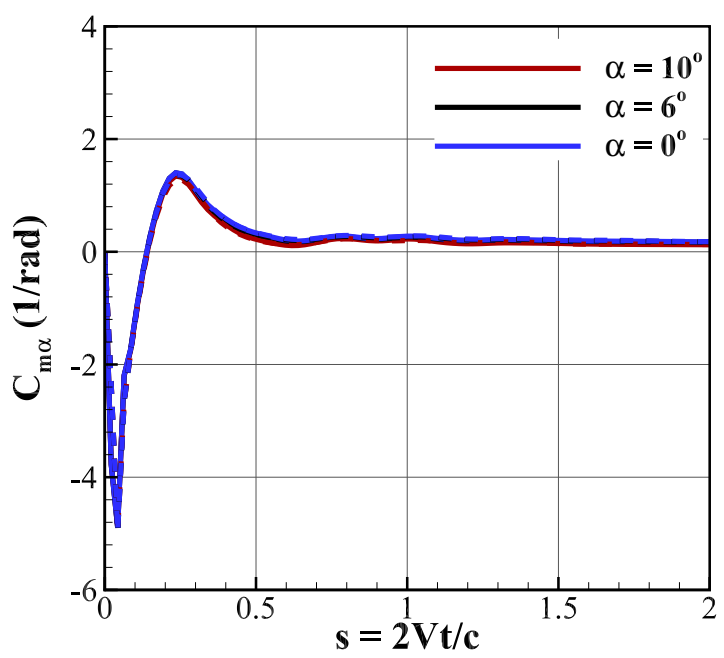

(d) nonlinear $C_{m_{\alpha}}$ functions ( $\left.M=0.15\right)$

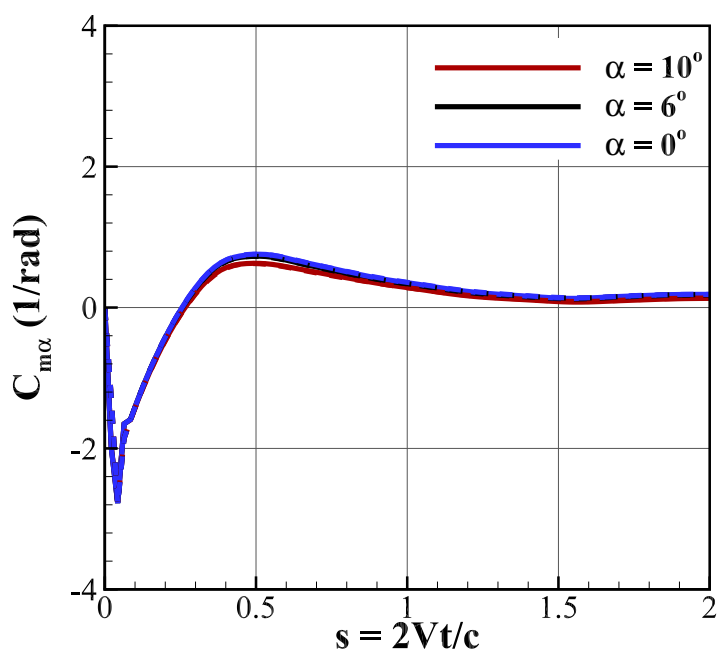

(f) nonlinear $C_{m_{\alpha}}$ functions $(M=0.3)$

Fig. 24 The nonlinear lift and pitching moment indicial functions with a unit step change of angle of attack at different Mach numbers. USM3D (dashed) and FUN3D (solid). 


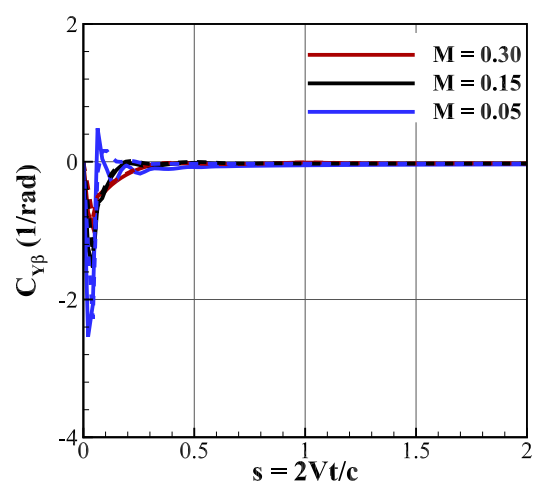

(a) linear $C_{Y_{\beta}}$ functions

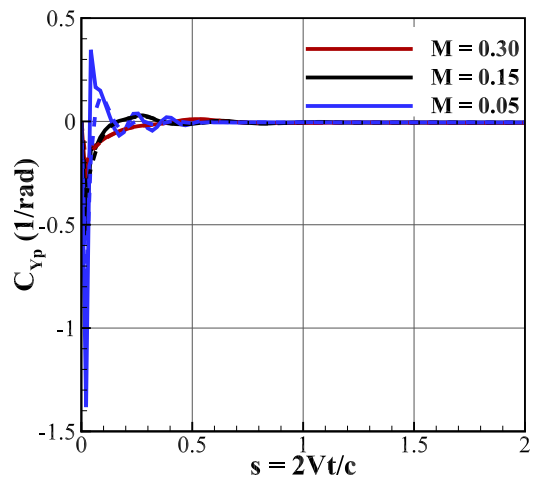

(d) linear $C_{Y_{p}}$ functions

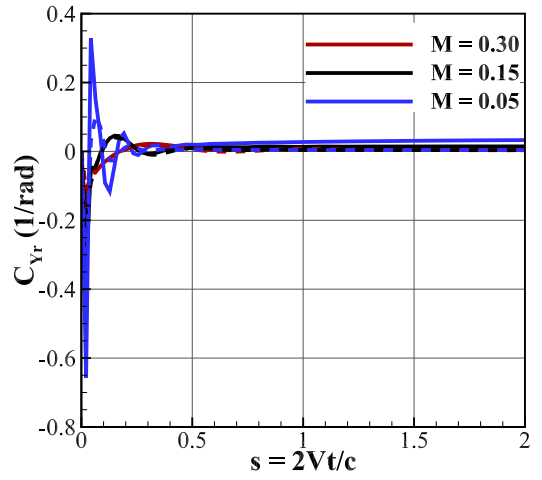

(g) linear $C_{Y_{r}}$ functions

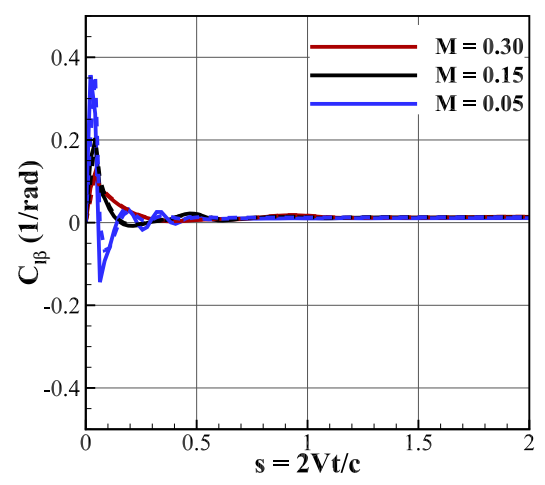

(b) linear $C_{l_{\beta}}$ functions

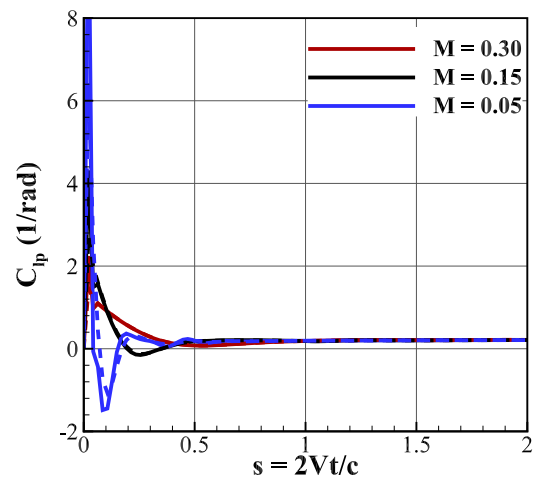

(e) linear $C_{l_{p}}$ functions

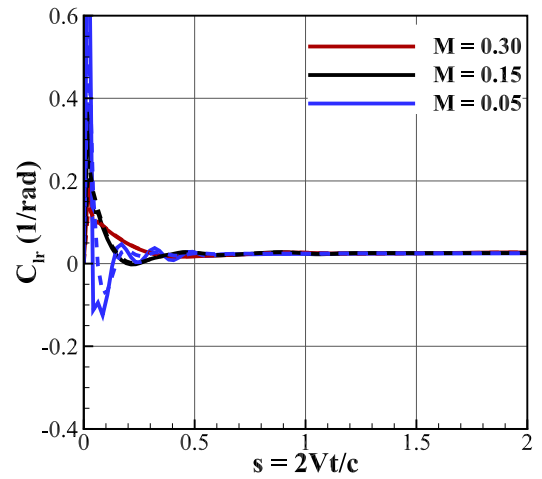

(h) linear $C_{l_{r}}$ functions

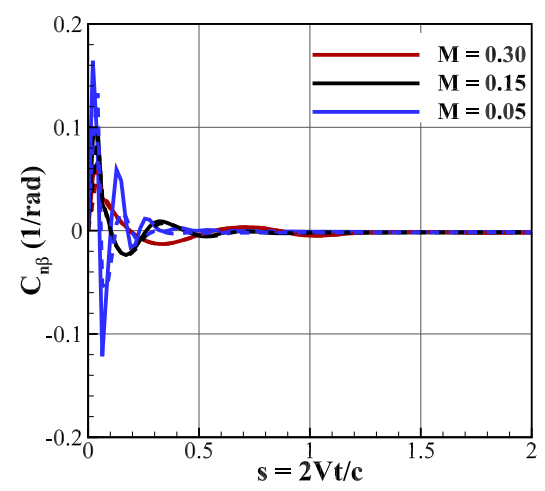

(c) linear $C_{n_{\beta}}$ functions

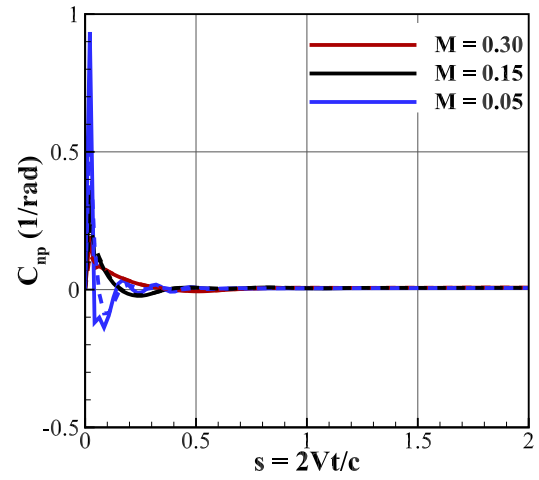

(f) linear $C_{n_{p}}$ functions

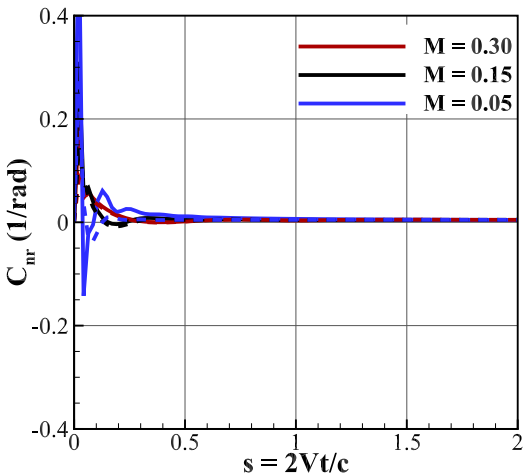

(i) linear $C_{n_{r}}$ functions

Fig. 25 The linear side-force, rolling moment, and yawing moment indicial functions with a unit step change of sideslip angle (a-c), normalized roll rate (d-f), and normalized yaw rate (g-i). USM3D (dashed) and FUN3D (solid). 


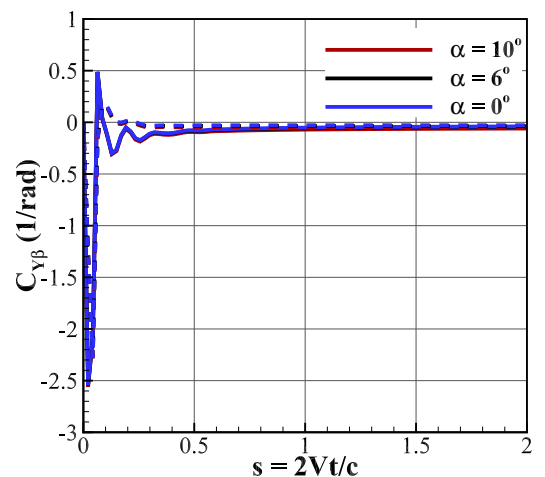

(a) nonlinear $C_{Y_{\beta}}$ functions $(M=0.05)$

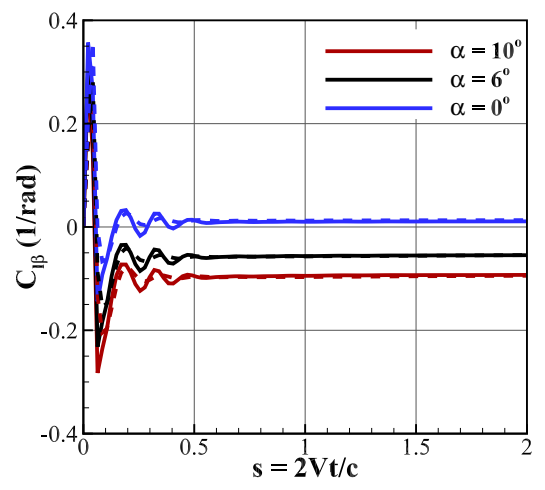

(d) nonlinear $C_{l_{\beta}}$ functions ( $M=0.05$ )

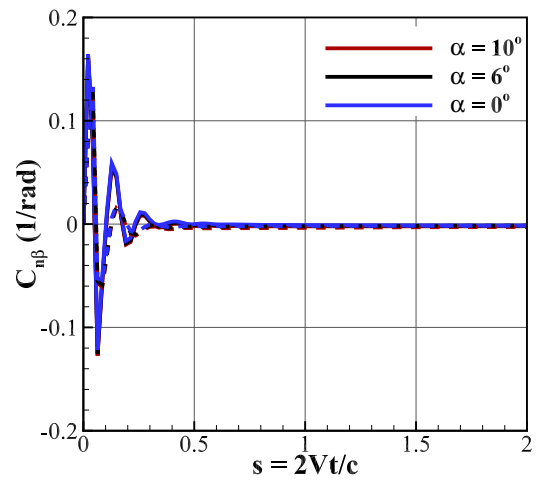

(g) nonlinear $C_{n_{\beta}}$ functions ( $M=0.05$ )

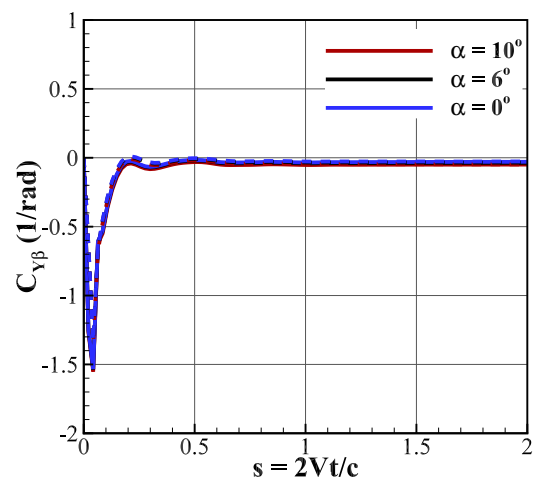

(b) nonlinear $C_{Y_{\beta}}$ functions $(M=0.15)$

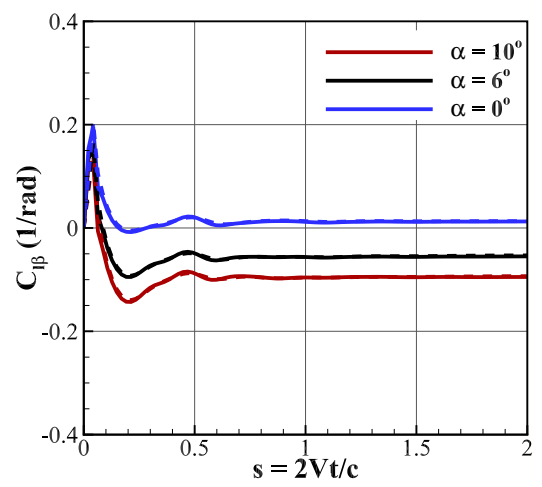

(e) nonlinear $C_{l_{\beta}}$ functions $(M=0.15)$

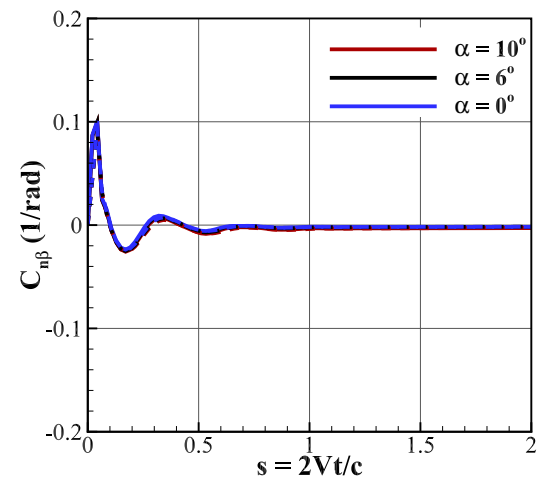

(h) nonlinear $C_{n_{\beta}}$ functions ( $M=0.15$ )

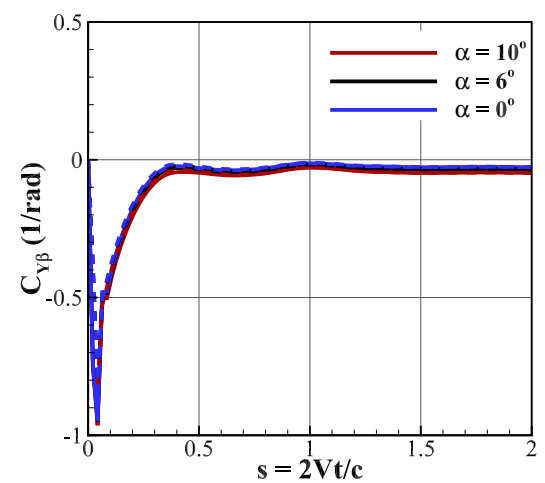

(c) nonlinear $C_{Y_{\beta}}$ functions $(M=0.3)$

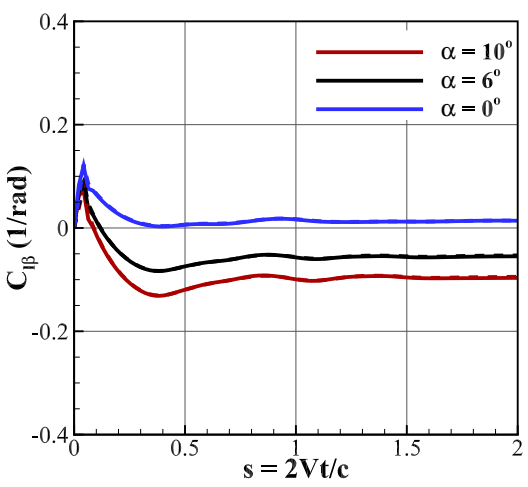

(f) nonlinear $C_{l_{\beta}}$ functions ( $M=0.3$ )

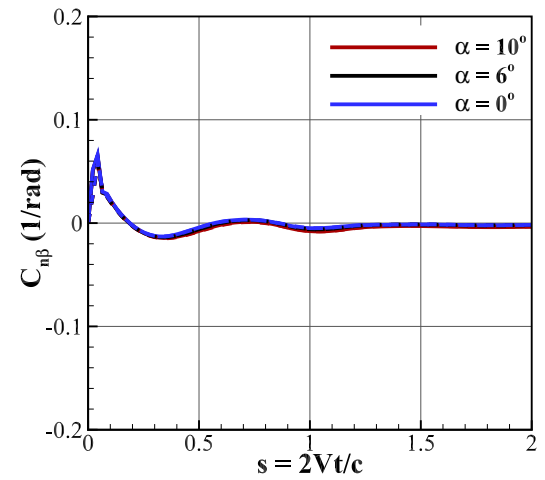

(i) nonlinear $C_{n_{\beta}}$ functions ( $M=0.3$ )

Fig. 26 The nonlinear side-force, rolling moment, and yawing moment indicial functions with a unit step change of sideslip angle at different Mach numbers. USM3D (dashed) and FUN3D (solid). 